\title{
DyNAMICAL EVOLUTION OF INFORMATION AND ENERGY in Causal Dispersive Media
}

by

Ahmed H. Dorrah

A thesis submitted in conformity with the requirements

for the degree of Master's of Applied Science.

Graduate Department of Electrical and Computer Engineering

University of Toronto

(c) Copyright 2015 by Ahmed H. Dorrah 


\title{
Abstract \\ Dynamical Evolution of Information and Energy in Causal Dispersive Media
}

\author{
Ahmed H. Dorrah \\ Master's of Applied Science. \\ Graduate Department of Electrical and Computer Engineering \\ University of Toronto \\ 2015
}

Energy exchange between an electromagnetic pulse and dispersive media may result in complicated, yet interesting, phenomena in which the group velocity becomes abnormal (i.e. superluminal or negative). For such cases, signal velocity (velocity of detectable information) remains debatable. In this thesis, we present a systematic study that can be applied to pulse propagation in any dispersive medium in order to quantify the detectable information content and calculate its speed at different propagation distances while accounting for: pulse reshaping effects, noise generated in the medium, and the detector. Accordingly, we present an operational context within which the constraints of superluminal signaling and its potential applications are shown. Furthermore, we explain the fundamental limitations imposed by the cut-off medium length beyond which superluminal propagation can not be achieved. Such analysis explores the fundamental limitations and capabilities of a broad range of fast (and slow) light applications. 


\section{Acknowledgements}

I would like to express my deepest gratitude to my research supervisor Professor Mo Mojahedi. Indeed, the successful completion of this thesis leveraged on his effective advice, continuous support, and professional guidance throughout the different stages of my work.

I would also like to thank the members of my thesis committee, Professor Costas D. Sarris and Professor J. Stewart Aitchison, for their thorough review of my thesis, fruitful discussion, and constructive feedback. In addition, I am grateful to Professor Ding Yuan for administrating the final presentation in a smooth way.

The support of my colleagues and friends in the ECE department had, in fact, a positive impact on the completion of this thesis. It also provided me with the motivation to keep going. For this reason, I would like to thank my fellow graduate students for providing both a friendly environment in addition to many useful technical discussions as well.

Finally, thank you very much my dear family for your sincere love and support. Without this, I would have never reached where I am right now. Thanks again! 


\section{Contents}

\begin{tabular}{lll}
\hline & Introduction & 1
\end{tabular}

1.1 Background . . . . . . . . . . . . . . . . . . . . . 1

1.2 Motivation . . . . . . . . . . . . . . . . . . . . . . . 3

1.2.1 How to quantify the velocity of detectable information in fasterthan-light (superluminal) pulses? . . . . . . . . . . . . 3

1.2.2 What is the physical mechanism of superluminal pulse transition to the subluminal regime? . . . . . . . . . . . . . . . . . 5

1.2.3 Can Information be entirely associated with non-analytic points (other than the front) of the pulse? . . . . . . . . . . . . 6

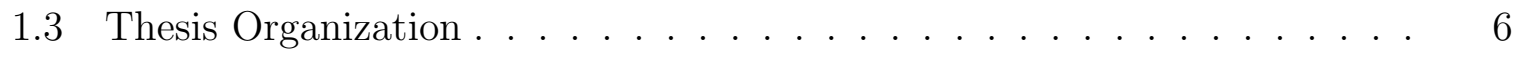

\begin{tabular}{|lll}
2 & Velocity Definitions in Dispersive Media & 9
\end{tabular}

$2.1 \quad$ Various velocity terms for a propagating electromagnetic pulse . . . . . . 9

2.1 .1 Phase Velocity . . . . . . . . . . . . . . . . . . . . 10

2.1 .2 Group Velocity . . . . . . . . . . . . . . . . . . . . 10

2.1 .3 Energy Transport Velocity . . . . . . . . . . . . . . . . . . 11

2.1 .4 Front Velocity . . . . . . . . . . . . . . . . . . 12

2.1 .5 Information Velocity $\ldots \ldots \ldots$. . . . . . . . . . . . . . 13

2.2 Connection between Velocity and Delay . . . . . . . . . . . . . . . . . . . 13

$2.2 .1 \quad$ Phase Delay . . . . . . . . . . . . . . . . . . . . . . . 13

2.2 .2 Group Delay . . . . . . . . . . . . . . . . . . . . . 14

2.3 Representation of Dispersion Terms . . . . . . . . . . . . . . . . . . . . . 14 
3 Method of Steepest Descent in Causal Dielectrics 16

3.1 Lorentzian Media . . . . . . . . . . . . . . . . . . . . . . . . . . 16

3.2 Method of Steepest Descent . . . . . . . . . . . . . . . . . . . . . . . 19

4 Physical Dynamics in Superluminal Pulse Propagation 25

4.1 Introduction . . . . . . . . . . . . . . . . . . . . . . . . . . . . 25

4.2 Double Resonance Lorentzian Media with Gain . . . . . . . . . . . . . . 27

$4.3 \quad$ Steepest Descent Analysis for Pulse propagation in a Gain medium . . . 34

4.4 Physical Dynamics of a Superluminal Pulse at Longer Propagation Lengths 36

4.5 Physical Dynamics of a Superluminal Pulse at different spectral widths . 42

4.6 Conclusion $\ldots \ldots \ldots$. . . . . . . . . . . . . . . . . . . . . . . . . . . . . 48

5 Velocity of Detectable Information in Faster-than-light Pulses 49

5.1 Introduction . . . . . . . . . . . . . . . . . . . . . 49

5.2 Literature shortcomings . . . . . . . . . . . . . . . . 51

5.3 Approach . . . . . . . . . . . . . . . . . . . . 52

$5.4 \quad$ Field Calculation in Causal Dispersive Medium . . . . . . . . . . . . . . 53

$5.5 \quad$ Signal Velocity in an Inverted Medium with Superluminal Delay . . . . . 57

5.6 Discussion . . . . . . . . . . . . . . . . . . . . 60

5.7 Conclusion . . . . . . . . . . . . . . . . . . 63

6 Detectable Information and Discontinuities in Causal pulses 65

6.1 Introduction . . . . . . . . . . . . . . . . 66

6.2 Approach . . . . . . . . . . . . . . . . . . . . 6 6 67

6.3 Field Propagation in Causal Slow-light Medium . . . . . . . . . . . . . . 68

6.4 Propagation of Discontinuities in Causal Slow-light Medium . . . . . . . 71

6.5 Arrival of Pulse Discontinuities and Detectable Information in Slow-Light Media ............................. 73

6.6 Propagation of Information in Causal Superluminal Medium . . . . . . . 76

6.7 Conclusion . . . . . . . . . . . . . . . . . . . . . . . . . . . . 79

\begin{tabular}{lll}
\hline & Conclusion and Future Work & 80
\end{tabular}

7.1 Future work . . . . . . . . . . . . . . . . . . . . . . . . 81 
7.1.1 Experimental demonstration of superluminal pulse transition to a subluminal regime ................... . . 81

7.1 .2 The role of the detector in information velocity . . . . . . . . . . 82

7.1.3 Utilizing the information capacity of superluminal media using advanced modulation techniques . . . . . . . . . . . . . . . 82

$\begin{array}{lll}8 & \text { Refereed Publications } & 84\end{array}$

8.1 Articles published in refereed journals: . . . . . . . . . . . . . . . . . 84

8.2 Articles presented in refereed conferences: . . . . . . . . . . . . . . . . . 84

\begin{tabular}{|l|l}
\hline Appendices & 85
\end{tabular}

\begin{tabular}{|lr}
\hline A FDTD Update Equations & 86
\end{tabular}

B Derivation of Superluminal-to-Subluminal Cutoff Distance 89

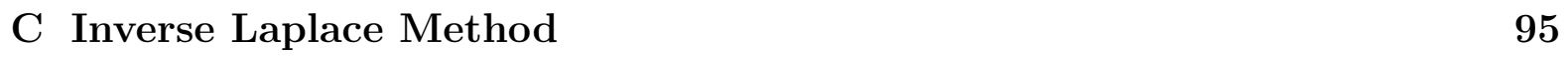

\begin{tabular}{ll}
\hline Bibliography & 97
\end{tabular} 


\section{List of Tables}

3.1 Numerical values for the parameters of the passive single resonance Lorentzian medium. . . . . . . . . . . . . . . . . . . 17

$4.1 \quad$ Numerical values for the parameters of the double resonance Lorenztian medium with gain (ammonia vapor cells). . . . . . . . . . . . . . . . . . 28

6.1 Numerical values for the parameters of the single resonance Lorenztian

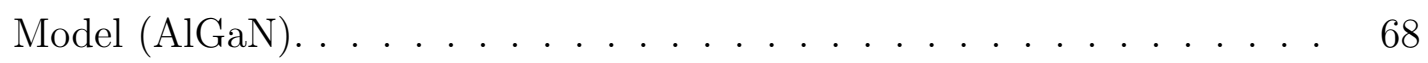




\section{List of Figures}

3.1 Real and imaginary parts of $n(\omega)$ as a function of frequency. Clearly, there exists a region of anomalous dispersion around the resonance frequency $\omega_{0}=4 \times 10^{16}\left(\mathrm{sec}^{-1}\right)$. . . . . . . . . . . . . . . . . 18

3.2 Frequency domain behavior of the passive Lorentzian medium. The medium

\begin{tabular}{|c|}
\hline absorption and group delay are calculated for a propagation distance equal \\
\hline to $0.25(\mu \mathrm{m})$. The blue dashed line corresponds to the luminal group delay \\
\hline
\end{tabular}

3.3 Path of the saddle points of $\phi\left(\omega, \theta^{\prime}\right)$ as a function of $\theta^{\prime}$ at a distance $L=$ $0.25(\mu \mathrm{m})$ inside the Lorentzian medium. The colored contours illustrates the $\operatorname{Re}\left[\phi\left(\omega, \theta^{\prime}\right)\right]$ evaluated at $\theta^{\prime}=0.636$ (which corresponds to time $t=$ $L / c) .\left(\omega_{+}, \omega_{+}^{\prime}\right)$ and $\left(\omega_{-}, \omega_{-}^{\prime}\right)$ signify the branch cuts (poles and zeroes) of the medium. . . . . . . . . . . . . . . . . . 21

3.4 Time domain response of the propagating field as a function of the dimen\begin{tabular}{|c|}
\hline sionless space-time parameter $\theta(\theta=c t / z)$. Cases (a-d) corresponds to \\
\hline
\end{tabular} propagation distance $0.25,0.5,0.75$ and $1(\mu m)$, respectively. . . . . . . 23

$4.1 \quad$ Index of refraction as a function of detuning, the left axis represents the real part and the right axis represents the imaginary part of $n(\omega)$, respectively. . . . . . . . . . . . . . . . . . . . . 28

4.2 Group Delay $\tau_{g}$ as a function of frequency at a distance $25(\mathrm{~cm})$ plotted against the normalized initial pulse spectrum. . . . . . . . . . . . . . 29 
$4.3 \quad$ Spectral width and medium gain. (a) The medium gain $g(\omega)$ for length $L=25(\mathrm{~cm}), 44.5(\mathrm{~cm}), 54(\mathrm{~cm})$ and $63(\mathrm{~cm})$ and a Gaussian pulse with temporal width $2 T=0.9(n s)$. (b) Medium gain $g(\omega)$ for a length $L=25$ $(\mathrm{cm})$ and pulse widths that correspond to $2 T=0.9(\mathrm{~ns}), 0.7(\mathrm{~ns}), 0.5(\mathrm{~ns})$ and $0.3(n s)$, respectively. (c) The medium gain $g(\omega)$ at a fixed length $L$ $=25(\mathrm{~cm})$ and pulse width $2 T=0.9(\mathrm{~ns})$ for oscillator strengths $\omega_{p}$ that is equal to $4 \delta, 4.8 \delta, 5.6 \delta$ and $6.4 \delta$, respectively . . . . . . . . . . . . . . . 31

4.4 Comparison between the exact calculations of the cutoff distance versus the approximate closed form expression when: (a) The input spectral width is increased (or as the temporal pulse width is reduced). (b) The medium resonances $\left(\omega_{0,2}-\omega_{0,1}\right)$ are tuned. (c) The plasma frequency (dispersion strength) is increased. . . . . . . . . . . . . . . . 33

4.5 Path of the saddle points $\omega_{S P}$ of $\phi\left(\omega_{S P}, \theta^{\prime}\right)$ as $\theta^{\prime}$ increases at propagation distance $25(\mathrm{~cm})$ inside the ammonia vapor cells. The colored (green) contours show $\left[R e\left\{\phi\left(\omega, \theta^{\prime}\right)\right\}\right]$ at $t=L / c . \ldots \ldots$. . . . . . . . . 37

4.6 Path of the saddle points $\omega_{S P}$ of $\phi\left(\omega_{S P}, \theta^{\prime}\right)$ as $\theta^{\prime}$ increases at propagation distance $44.5(\mathrm{~cm})$ inside the ammonia vapor cells. The colored (green) contours show $\left[R e\left\{\phi\left(\omega, \theta^{\prime}\right)\right\}\right]$ at $t=L / c$. . . . . . . . . . . . . . . 37

4.7 Path of the saddle points $\omega_{S P}$ of $\phi\left(\omega_{S P}, \theta^{\prime}\right)$ as $\theta^{\prime}$ increases at propagation distance $54(\mathrm{~cm})$ inside the ammonia vapor cells. The colored (green) contours show $\left[R e\left\{\phi\left(\omega, \theta^{\prime}\right)\right\}\right]$ at $t=L / c$. . . . . . . . . . . . . . . 38

4.8 Path of the saddle points $\omega_{S P}$ of $\phi\left(\omega_{S P}, \theta^{\prime}\right)$ as $\theta^{\prime}$ increases at propagation \begin{tabular}{|c|}
\hline distance $63(\mathrm{~cm})$ inside the ammonia vapor cells. The colored (green) \\
\hline
\end{tabular}

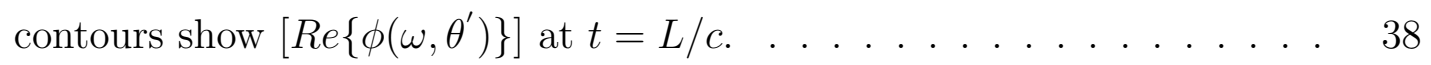

4.9 Saddle point contributions. The left column corresponds to the contribution of $\left(\omega_{S P, M}\right)$ and the right column corresponds to the contribution of $\left(\omega_{S P, L}\right)$. Cases (a-d) refer to propagation length of 25, 44.5, 54 and 63 (cm) inside the ammonia vapor cells, respectively. . . . . . . . . . . . . . 40

4.10 The output field $A(L, t)$. The black dotted curves correspond to the case of vacuum and $\langle t\rangle$ denotes the pulse arrival time. Cases (a-d) refer to propagation lengths of $25,44.5,54$ and $63(\mathrm{~cm})$, respectively. . . . . . . 
4.11 Path of saddle points $\omega_{S P}$ as $\theta^{\prime}$ increases at a fixed propagation distance $=25(\mathrm{~cm})$ inside the ammonia vapor for Gaussian pulse with an initial pulse width $2 \mathrm{~T}=0.9(n s) . \ldots \ldots \ldots \ldots$

4.12 Path of saddle points $\omega_{S P}$ as $\theta^{\prime}$ increases at a fixed propagation distance $=25(\mathrm{~cm})$ inside the ammonia vapor for Gaussian pulse with an initial pulse width $2 \mathrm{~T}=0.7(n s) . \ldots \ldots \ldots \ldots$

4.13 Path of saddle points $\omega_{S P}$ as $\theta^{\prime}$ increases at a fixed propagation distance $=25(\mathrm{~cm})$ inside the ammonia vapor for Gaussian pulse with an initial pulse width $2 \mathrm{~T}=0.5(n s) . \ldots \ldots$. . . . . . . . . . . . . . 44

4.14 Path of saddle points $\omega_{S P}$ as $\theta^{\prime}$ increases at a fixed propagation distance $=25(\mathrm{~cm})$ inside the ammonia vapor for Gaussian pulse with an initial pulse width $2 \mathrm{~T}=0.3(n s) . \ldots \ldots \ldots$. . . . . . . . . . 44

4.15 The decomposed contribution of the saddle point of the phase function $\phi\left(\omega, \theta^{\prime}\right)$. The left column corresponds to the contribution of the middle saddle points $\left(\omega_{S P, M}\right)$ and the right column corresponds to the contribution of the Left saddle points $\left(\omega_{S P, L}\right)$. Cases $(\mathrm{a}-\mathrm{d})$ refer to initial pulse width $2 \mathrm{~T}=0.9(n s), 0.7(n s), 0.5(n s)$, and $0.3(n s)$, respectively. . . . . . . . 46

4.16 The total output field response at a propagation distance $25(\mathrm{~cm})$ for the case of an AGD medium (ammonia vapor). The field is obtained by adding the contributions of $\omega_{S P, L}, \omega_{S P, M}$ and $\omega_{S P, R}$. The black dotted curves correspond to the case of vacuum and $\langle t\rangle$ denotes the pulse arrival time. Cases (a),(b),(c) and (d) refer to initial pulse width $2 \mathrm{~T}=0.9$ $(n s), 0.7(n s), 0.5(n s)$ and $0.3(n s)$, respectively. . . . . . . . . . . . .

5.1 Index of refraction $n(\omega)$ and group delay as a function of detuning (the blue dashed line denotes the luminal group delay in vacuum). The vertical arrows mark the spectral width of the input pulse. . . . . . . . . . . . . . 54 
5.2 Path of the saddle points of $\left[\phi\left(\omega, \theta^{\prime}\right)\right]$ as a function of $\theta^{\prime}$ at a distance $L$ $=25(\mathrm{~cm})$ inside the ammonia vapor. The colored contours illustrates the $\operatorname{Re}\left[\phi\left(\omega, \theta^{\prime}\right)\right]$ evaluated at $\theta^{\prime}=-0.45$ (which corresponds to time $t=$ $L / c)$. The first and second resonances are denoted by $\left(\omega_{0,1}\right)$ and $\left(\omega_{0,2}\right)$, respectively. $\left(\omega_{+}{ }^{(0)}, \omega_{+}{ }^{(1)}\right)$ and $\left(\omega_{+}{ }^{(2)}, \omega_{+}{ }^{(3)}\right)$ signify the branch cuts for the resonances $\omega_{0,1}$ and $\omega_{0,2} . \ldots \ldots \ldots$. . . . . . . . . . 55

5.3 Normalized medium response at propagation distance $L=25(\mathrm{~cm})$. The insets depict the arrival of the pulse front after duration $L / c$ in compliance with Einstein's causality. . . . . . . . . . . . . . . . . . . . . . . . 56

5.4 Relative signal delay in ammonia vapor cells with respect to a vacuum channel as a function of propagation distance in units of $(c / \delta)$. The relative signal delay is compared with the corresponding relative group delay (denoted by the blue line). This comparison is carried out for three different noise levels at the detector side. . . . . . . . . . . . . . . . . . . . . . 59

5.5 Signal-to-noise ratio as a function of time at a propagation depth of 25 $(\mathrm{cm})$ in the ammonia vapor. The inset shows the information window at vicinity of the detection threshold as compared to a vacuum channel of the same length. . . . . . . . . . . . . . . . . . . . .

$5.6 \quad S N R(L, t)$ at different propagation depths $L=2.083(c / \delta), 2.5(c / \delta)$ and $2.917(\mathrm{c} / \delta)$ (which corresponds to 25,30 and $35(\mathrm{~cm})$, respectively) in an AGD medium and compared to a vacuum channel. Evidently, information velocity is retarded in an AGD medium despite the exhibited superluminal behavior indicated by the center of mass calculations. . . . . . . . . . 63

6.1 Input and output pulses of a negative group delay band pass amplifier. The discontinuity "kicks" the amplifiers, producing large-amplitude ringing. This ringing exceeds the scale of the graph and appears as vertical lines following the discontinuity. Input and output were acquired simultaneously with no averaging. Curves have been offset vertically for clarity [32]. . . . . . . . . . . . . . . . . . . . . 66

6.2 Real and imaginary parts of the index of refraction of AlGaN. . . . . . . 69 
6.3 The input Gaussian spectrum is superimposed on the group delay $\left(\tau_{g}\right)$ plot. $\tau_{g}$ is calculated for a length $L=30(\mu m)$. The blue dashed line corresponds to $\tau_{g}$ in vacuum. . . . . . . . . . . . . . . . . . . . . . . . . 69

6.4 Pulse propagation in a medium with subluminal group delay $\tau_{g}$ and vacuum at a distance $L=30(\mu m)$. Clearly, the pulse peak in the slow medium is delayed by the amount of $0.1133(\mathrm{ps})$ with respect to the vacuum channel. . . . . . . . . . . . . . . . . . . . . . . . . . . . . 70

6.5 Output response of the medium when excited by a truncated pulse. (a) After propagation in a slow light medium of a distance $30(\mu m)$. (b)After propagation in a vacuum channel with the same length. . . . . . . . . . . 72

6.6 (a)Arrival of the detectable information within the window of discontinuities. (b)The strictly luminal behavior of the discontinuities (front and pulse truncation) in the slow-light medium and vacuum. . . . . . . . . . 74

6.7 (a)Arrival of the detectable information within the window of discontinuities. (b)The strictly luminal behavior of the discontinuities (front and pulse truncation) in the slow-light medium and vacuum. . . . . . . . . . 75

6.8 The superluminal medium response in the frequency and time domains. (a)Input pulse spectrum fitted over the group delay plot (as a function of frequency) of a superluminal medium. The black dashed lines correspond to group delay in vacuum. (b)Comparison of the time domain response of the superluminal medium and a vacuum channel after propagation distance $L=2.5(\mu m)$. . . . . . . . . . . . . . . . . . . . . . . . . . . 77

6.9 The input spectrum fitted over the group delay plot for the case of a superluminal medium. The black dashed lines correspond to group delay in vacuum. . . . . . . . . . . . . . . . . . . . . . . . . . 78 


\section{Nomenclature}

$\delta \quad$ Phenomenological line-width of Lorentzian medium.

$\Gamma \quad$ Pulse compression factor.

$\hbar \quad$ Planck's contsant $\hbar=6.626 \times 10^{-34} \mathrm{~kg} / \mathrm{s}$.

$\langle t\rangle \quad$ Expectation of the arrival time of the pulse.

$\omega_{0} \quad$ Resonance frequency of Lorentzian medium.

$\omega_{p} \quad$ Plasma frequency of Lorentzian medium.

$\omega_{S P} \quad$ Saddle point frequency.

$\tau_{g} \quad$ Group delay.

$\tau_{p} \quad$ Phase delay.

$\theta^{\prime} \quad$ Dimensionless space time parameter.

$A G D$ Abnormal group delay.

$B_{e} \quad$ Detection bandwidth.

$B_{o} \quad$ Optical bandwidth.

c Speed of Light in Vacuum in units of $\mathrm{m} / \mathrm{sec}$.

$E(z, t)$ Electromagnetic field at an arbitrary space-time point.

G Photo-multiplier detector gain.

$g(\omega)$ Lorentzian medium gain as a function of frequency.

$G_{e} \quad$ Equivalent Shear Modulus 
$G D D$ Group delay dispersion.

$i_{d} \quad$ Dark current in the detector circuit.

$L \quad$ Dispersive medium length in units of $\mathrm{m}$.

$n(\omega)$ Index of refraction as a function of frequency.

$N G D$ Negative group delay.

$P_{A S E-A S E}(t)$ Self-beat noise of the amplified spontaneous emission.

$P_{A S E}(t)$ spontaneous emission noise.

$P_{\text {Shot }}(t)$ Shot noise power.

$P_{\text {Sig-ASE }}(t)$ Mutual beat noise between the signal and amplified spontaneous emission.

$S \quad$ Poynting vector.

$S N R$ Signal-to-Noise ratio.

$u(z,-\infty)$ Energy initially stored in the medium before excitation.

$u(z, t)$ Total energy density.

$u(z, t)_{\text {exchange }}$ Energy exchanged between the field and the medium.

$u(z, t)_{\text {field }}$ Energy stored in the field.

$v_{e} \quad$ Velocity of energy transport

$v_{G} \quad$ Group velocity.

$v_{p} \quad$ Phase velocity. 


\section{Chapter 1}

\section{Introduction}

In a temporally dispersive medium - in which the index of refraction is a function of frequency - different spectral components of an Electromagnetic pulse propagate at different phase velocities and can be non-uniformly attenuated (in passive media) or amplified (in active media) depending on the frequency of each component. As these weighted spectral components propagate in the medium, they add up constructively or destructively in some points of space in such a way that leads to reshaping of the pulse envelope. In general, this would lead to signal distortion; however, by carefully designing some parameters - such as the medium dispersion characteristics, the input spectrum, carrier frequency, and propagation distance - the output pulse peak can be advanced in time as compared to a companion pulse traveling the same distance in vacuum. On the other hand, the interrelated phase delay and scaling may lead to a scenario in which the pulse peak is significantly slowed down. Consequently, such dispersion effects can be utilized for various applications including fast signaling, optical data storage and interconnect design. In this introductory chapter, a brief history on dispersive media and its applications is given and the main scope of the thesis is presented.

\section{$1.1 \quad$ Background}

Extensive research efforts have been carried out to create materials with abnormal dispersion behavior. Dispersive media are characterized by having a non-constant index of refraction that is a function of the frequency components of the propagating wave. 
An electromagnetic pulse or wavepacket - propagating in such a dispersive mediumcan be regarded as a superposition of many monochromatic waves each propagating with a different phase velocity. Depending on the pulse spectrum, carrier frequency, propagation distance, and the medium dispersion strength, the wavepacket typically experiences reshaping during propagation. Under some cleverly designed configurations, it is observed that the peak of the pulse propagating in a dispersive medium may travel faster than the peak of a companion pulse traversing the same distance in free space - an effect called superluminal group velocity (SGV). Under other circumstances, the peak of the transmitted pulse (output peak) may even be detected before the peak of the input pulse interacts with the medium - a phenomenon known as negative group velocity (NGV). From a fundamental point-of-view, both SGV and NGV describe the same phenomenon which can be collectively referred-to as abnormal group velocities (AGVs).

The counter intuitive phenomenon of AGV was first investigated long ago by Sommerfeld [1] and Brillouin [2, 3] by studying the transients associated with electromagnetic pulse propagation in absorbing causal dielectrics. Afterwards, Garret and McCumber were able to establish the theoretical possibility of achieving superluminal propagation with minimal pulse distortion and they pointed out to the full compliance of this phenomenon with Einstein's causality [4]. Later on, Chu and Wong successfully realized AGV in an experimental setup using laser pulses [5]. Then afterwards, Segard and Macke extended the experiment to the millimeter waves [6].

More recently, AGV has been observed in passive and active media: using a single microwave pulse [7, 8, 9], in under-sized waveguides [10, 11, 12, 13], in electronic circuits [14], at optical frequencies [15, 16, 17, 18, 19], in the single-photon limit [20, 22, 23, 24, in negative index meta-materials [25, 26], using ring resonators [27, 28], and by encoding information in the spatial degrees of freedom of an optical pulse [29]. Despite its counter intuitive implications, AGV has been considered to be in full agreement with the requirements of special relativity (Einstein causality) which prohibits information to be transmitted faster than speed of light in vacuum $(c)$ [30, 31, 32, 33, 34, 35, 36, 37.

The common line of arguments asserts that any causal pulse used for signaling must have a transitional turn-on in time and space, referred to as the "front", which can be represented mathematically as non-analytic point(s) in the complex frequency domain 
[22, 38. As it has been shown by Sommerfeld and Brillouin long ago, the front and the earliest oscillations associated with it-referred to as precursors or forerunners-always travel luminaly [1, 2, 3]. As such, information and the velocity by which it travels must be associated with these points of non-analyticity and the velocity by which it propagates - a velocity which is never superluminal.

Furthermore, even for the cases in which superluminal propagation is observed, it has been shown that the pulse (typically a smoothly varying pulse) behavior could have been analytically extrapolated from earlier time samples detected at the medium output [31, 32, 33. Accordingly, information velocity should not be tied to the superluminal effect of the group velocity [31, 35, 36, 38]

\subsection{Motivation}

Although several studies have investigated pulse propagation with abnormal (superluminal or negative) group velocities, and the compliance of this phenomenon to the fundamental requirements of Einstein causality; however, the literature still does not have conclusive answers to many fundamental questions. Addressing these questions will showcase the capabilities as well as the fundamental limitations that should be taken into account when utilizing abnormal media in applications for fast signaling and information transfer.

These questions are stated in the following subsections and it is the goal of the current thesis to address these shortcomings.

\subsubsection{How to quantify the velocity of detectable information in faster-than- light (superluminal) pulses?}

Although it has been well established that genuine information is encoded in the strictly luminal points of non analyticity at the onset of the pulse (the front); however, in many practical situations, detectable information can not be entirely associated with the arrival of the pulse front due to the following:

First, the field strength associated with the front and precursor oscillations is extremely small and well below the detection level[31]. This makes the detection of the 
points of non-analyticity (singularities) if not impossible, certainly very challenging. In fact, when such singularities have been observed to demonstrate the compliance of AGD with Einstein causality [16], special care in generating the singularities and their detection has been employed. Accordingly, relating the information velocity to the strictly luminal front is practically non meaningful.

Second, information cannot be viewed as a localized quantity (e.g. points of non analyticity alone) as its detection requires the act of a detector and a window of time associated with arrival of enough power (sufficient amount of photons) to trigger a detection process.

Third, information, its propagation, and its detection are stochastic processes, in which each subsystem of a communication system plays an important role. Each subsystem introduces a certain amount of noise that imposes considerable effects on the stochastic nature of the information and its velocity. In short, any definition for detectable information should account for the associated noise from the channel and the non-ideal detector.

In light of the three mentioned considerations, there is no universal systematic approach that can quantify the velocity of detectable information of an electromagnetic pulse propagating in a general dispersive medium.

Although some efforts have been made to investigate the behavior of information in superluminal pulses [16, 17, 29, 39, 40] and to address some of the above shortcomings, the results are still inconclusive for many reasons:

First, the effect of the medium length — which has a direct impact on the dispersion characteristics of the medium and the reshaping of the pulse-has been widely ignored. In fact, signal velocity has always been studied at a fixed propagation distance instead of studying its evolution at different space points and thus the behavior of the signal velocity at different propagation distances is still unexplored.

Second, in the theoretical analysis [39, 40], pulse reshaping effects in the form of compression (or broadening) and non-uniform scaling have been neglected. Given the fact that pulse advancement can be in the order of only $3 \%$ of the initial pulse width in some cases [17, 39], pulse compression effects can significantly affect the interpretation of the results and cannot be overlooked. 
In this thesis, the velocity of information is calculated in a dispersive medium with gain while taking into account pulse reshaping effects, in addition to the classical and quantum noise sources associated with the medium and the non-ideal detector. Such noise sources play an important role in the stochastic behavior of information and its effect should be included in any definition that describes the evolution of detectable information.

Moreover, signal velocity is calculated at different space points to investigate the spatial evolution of information. These calculations are also applied to the case of propagation in a vacuum channel for comparison and in order to to verify the compliance to Einstein causality. The analysis provides a context within which:

1. The spatial behavior of information and its velocity is studied for the first time in the literature.

2. Cases in which information is detected in a superluminal medium faster than its detection in a vacuum channel of the same length (fast information transfer) are presented together with their proper interpretation.

3. Compliance to the fundamental requirements of Einstein causality, even for the case of "fast information transfer", is imposed without the need to refer to the undetectable front.

4. Three different points-of-view ${ }^{1}$ that have previously been discussed in the literature with regard to the possibility of superluminal signaling [3, 17, 29] are unified.

\subsubsection{What is the physical mechanism of superluminal pulse transition to the subluminal regime?}

The gain (absorption) and phase delay of any dispersive medium is function of its length. As such, the length of the medium plays an important role in the temporal behavior and reshaping effects of the pulse during propagation. Furthermore, there are fundamental limitations on the medium length that can prevent the superluminal effect. In fact, it has been recently shown that, for a superluminal medium, there always exists a cutoff distance beyond which a superluminal pulse becomes subluminal [41]. However, to the best of our knowledge, there does not exist a systematic approach in the literature that

\footnotetext{
${ }^{1}$ This will be discussed in details in section 5.6.
} 
actually explains the mechanism of this transition. Moreover, there is not a closed-form expression that predicts the cutoff distance. These shortcomings are addressed in the current thesis. We present a generic approach that can be applied to any dispersive medium in order to investigate the transition of a superluminal pulse to the subluminal regime. Using such approach, a superluminal pulse is decomposed into its superluminal and subluminal components and the energy distribution within these components is evaluated at different space points. The analysis illustrates how the center-of-mass of the pulse is time delayed as a result of being dominated by the subluminal components at longer distances. In addition, the analysis provides - for the first time in the literature - a closed form expression that relates superluminal-to-subluminal pulse transition to factors such as: the pulse width, propagation distance, and medium dispersion characteristics.

\subsubsection{Can Information be entirely associated with non-analytic points (other than the front) of the pulse?}

It has been argued that information can be encoded in the strictly luminal non-analytic points (unpredictable discontinuities other than the front) of the pulse [65, 66]. In this thesis, we present scenarios in which a pulse carries multiple discontinuities and the information content of the pulse remains below the detection threshold (not practically detectable) even after the arrival of all the non-analytic points of the pulse at the receiver. We thus argue that, from a practical point-of-view, information can not be entirely associated with the pulse discontinuities. This is demonstrated in details for the case of slow light media. Accordingly, the fundamental capabilities of applications involving optical storage and data buffers are discussed.

\subsection{Thesis Organization}

This thesis is organized as follows:

In chapter 2, five different velocity definitions - associated with propagation in temporally dispersive media - are introduced along with their connection with the more universal concept of delay. Afterwards, some prerequisite expressions for characterizing dispersion are presented in terms of the aforementioned velocities and delays expressions. 
In chapter 3 , we focus on the dispersion characteristics of casual dispersive media that can be described in the form of a Lorentzian function. For such media, the analytic expressions for calculating the propagating field-using the saddle point analysis (method of the steepest descent) - are introduced and the results are compared with the finite-difference time-domain method (FDTD). Such analysis would be utilized in the subsequent chapters.

In chapter 4 , the method of steepest descent is extended to the case of a doubleresonance Lorentzian medium with gain. Such medium can support superluminal propagation over a finite range of frequencies. In order to illustrate the fundamental limitations imposed by the medium length on superluminal propagation, the field is decomposed to its subluminal and superluminal components and the respective energy content is calculated at different propagation distances. It is found that there always exists a cutoff distance beyond which a superluminal pulse becomes subluminal. The physical mechanism of this transition is discussed.

Chapter 5 tackles the question of whether superluminal signaling is possible. The velocity of information is calculated for a double resonance gain medium in the presence of noise. Such calculation takes into account the classical and quantum generated noises in the medium and a non-ideal noisy detector in addition to considering pulse reshaping effects. Information velocity - which is related to the propagation speed of a point with a constant signal-to-noise ratio (SNR) - is extended to be a function of both space and time in order to study the temporal and spatial evolution of information. Cases in which the velocity of detectable information in the dispersive medium is time advanced compared to a corresponding vacuum channel are presented with their interpretation. Interestingly, based on this approach, it is shown that Einstein causality is always conserved without the need to refer to the practically undetectable front.

In chapter 6 , the distinction between the strictly luminal non-analytic points and the detectable information content of the pulse is demonstrated. Using truncated Gaussian 
pulses at the input of slow (and fast) light media, it is shown that the truncation point (pulse discontinuity) travels at a luminal speed. Nevertheless, the pulse discontinuity is not necessarily associated with the arrival of detectable information at the receiver side. The analysis thus provides a context in which the distinction between the points of non-analyticity, energy transport, and detectable information of a pulse can be understood.

Finally, the conclusion and potential future work is given in chapter 7 . 


\section{Chapter 2}

\section{Velocity Definitions in Dispersive}

\section{Media}

An electromagnetic pulse or wavepacket propagating in vacuum channel experiences a linear phase in space and time. For such a wavepacket, all the spectral components travel at the same speed $(c)$ after propagating a distance $L$ and the pulse preserves its temporal shape with a peak that is time shifted with duration $L / c$. In a temporally dispersive medium, however, things become more complicated and a wavepacket encounters at least five different velocities that are non necessarily equal (unlike the case of a vacuum channel). In this chapter the basic terminology needed to distinguish between these five velocity definitions is introduced, then the relation between these velocity definitions with regard to the more universal concept of delay is shown. These terms will collectively be used throughout the thesis in the subsequent chapters.

\subsection{Various velocity terms for a propagating electromagnetic pulse}

An electromagnetic pulse or wavepacket can be regarded as a superposition of many monochromatic waves or sinusoidal harmonics. The monochromatic waves are added together in such a way that constructive interference occurs at the position of the pulse peak, whereas destructive interference takes place at the pulse trails.

During propagation in a temporally dispersive medium, the monochromatic waves 
(or spectral components) of the pulse travel with different velocities. Accordingly, the propagating pulse may encounter reshaping and distortion.

In general, an electromagnetic pulse propagation in a temporally dispersive medium can be characterized by at least five different velocity definitions that are not necessarily identical. Those are: phase velocity, group velocity, front velocity, velocity of energy transport, and information velocity. In the following subsections we present a brief overview for each of the aforementioned velocity terms.

\subsubsection{Phase Velocity}

Phase velocity, denoted as $v_{p}$, is associated with the speed of a single monochromatic wave constructing the wavepacket. In other words, it is the speed of propagation of a point with a constant phase. The phase velocity is expressed as:

$$
v_{p}=\frac{\omega}{k}
$$

Where, $\omega$ is the frequency of the single monochromatic wave and $k$ is the wave number vector defined as

$$
k=\omega \frac{n(\omega)}{c}
$$

Here, $n(\omega)$ is the index of refraction of the medium and $c$ is the speed of light in vacuum. For propagation in a vacuum channel - in which $n(\omega)$ is constant and is equal to unity-

$v_{p}$ is equal to $c$ for all the spectral components of the wavepacket. However, in a general dispersive medium - in which $n(\omega)$ is a function of frequency - each monochromatic wave composing the wavepacket propagates with a different phase velocity. In such case, $v_{p}$ can either be greater than $c$, smaller than $c$ or even negative.

\subsubsection{Group Velocity}

Group velocity, denoted as $v_{g}$, is generally associated with the speed by which the pulse peak (or envelope) propagates. In a more formal way, for $1-\mathrm{D}$ propagation, $v_{g}$ is written as:

$$
v_{g}=\frac{d \omega}{d k}
$$


Equivalently,

$$
v_{g}=\frac{c}{n(\omega)+\omega \frac{d n(\omega)}{d \omega}}
$$

As such, in a medium with no dispersion - such as vacuum where the index of refraction $(n)$ is constant and equal to $1-v_{g}$ is equal to $c$ which is also equivalent to the phase velocity $v_{p}$. In a dispersive medium, however, $n(\omega)$ is a function of the excitation

frequency and the term $\frac{d n(\omega)}{d \omega}$ in the denominator of Eq. 2.4 which is also referred to as the group index $\left(n_{g}=n(\omega)+\omega \frac{d n(\omega)}{d \omega}\right)$ - may lead to scenarios in which $v_{g}$ can be greater than $c$, smaller than $c$ or even negative. Cases in which $v_{g}$ is greater than the speed of light in vacuum $(c)$ are referred to as superluminal group velocity; whereas cases in which $v_{g}$ is smaller than $c$ are referred to as subluminal group velocity. On the other hand, negative group velocity describes the counter intuitive phenomenon in which the output pulse peak is detected before the input pulse peak interacts with the medium.

It should be noted that associating the group velocity $v_{g}$ with the speed of the pulse peak is not entirely valid in some cases. For instance, in a medium with strong dispersion, the output pulse may suffer from severe distortion that its peak can no longer be distinguishable. In other cases, like double resonance media, the output pulse may even have more than one distinguishable peak. In such cases, the group velocity definition loses its meaning and it becomes more adequate to characterize the speed of propagation of the field by referring to its energy distribution and not just its peak (as discussed next).

\subsubsection{Energy Transport Velocity}

The concept of energy transport and its velocity was first investigated in the early 1900's by Sommerfeld and Brillouin. They pointed out to the possibility of attaining superluminal propagation in causal dispersive media with absorption. They defined the velocity of energy transport $\left(v_{e}\right)$ as the ratio between the propagating poynting flux and the energy density. This is expressed as [3]:

$$
v_{e}=\frac{\mathbf{S}}{u}
$$


Where, $\mathbf{S}$ is the poynting vector $\mathbf{S}=\mathbf{E} \times \mathbf{H}$ and $u$ is the energy density. At an arbitrary instant of time $t$ and propagation distance $z$, the energy density $u$ includes the energy stored in the field $u(z, t)_{\text {field }}$, the energy exchanged between the field and the medium $u(z, t)_{\text {exchange }}$ and the energy content initially stored in the medium before excitation $u(z,-\infty)$ [43]. Depending on the choices of energy densities in the denominator of Eq. 2.5, $v_{e}$ may become luminal [44] or superluminal [45]. In order to ensure strict luminality for $v_{e}$, the energy density in the denominator of Eq. 2.5 should at a minimum be equal to the energy of the field.

The energy density $u$ in the above definition, however, is not easily accessible in practical situations in which it is not clear how to measure $u(z, t)_{\text {exchange, instantaneously. }}$ Accordingly, a more recent definition for energy flow has been proposed in the literature [46]. This definition relies only on the Poynting flux which can be practically assessed using a detector. As such, the average energy flow of the field is regarded as the expectation of the arrival time of the Poynting vector which is described as

$$
\langle t\rangle=\frac{\hat{u} \cdot \int_{-\infty}^{\infty} t \mathbf{S}(\mathbf{z}, \mathbf{t}) d t}{\hat{u} \cdot \int_{-\infty}^{\infty} \mathbf{S}(\mathbf{z}, \mathbf{t}) d t} .
$$

The term $S(z, t)$ denotes the Poynting vector of the propagating field and $u$ is a unit vector along the normal direction with respect to the detector surface.

\subsubsection{Front Velocity}

The front velocity $\left(v_{f}\right)$ is associated with the speed of the singularities due to the transitional turn-on of the pulse in space and time. The signal front is mathematically represented by non-analytic points (singularities) in the complex frequency domain. As has been shown by Sommerfeld and Brillouinm, these singularities correspond to high frequencies which in effect propagate with a strictly luminal speed $(c)$ regardless of the medium type. Accordingly, the front velocity enforces the fundamental requirements of Einstein causality under all circumstances. 


\subsubsection{Information Velocity}

Information velocity $\left(v_{i}\right)$ is the most debated velocity definition with regard to propagation in dispersive media and represents the main theme of this study. Such velocity characterizes the speed of propagation of useful information in a communication channel. From a practical point-of-view, information is recoverable when the optical signal-to-noise ratio at the detector exceeds a certain threshold. Accordingly, $v_{i}$ is interchangeably used with the term signal velocity which is related to the speed of a point that exhibits a constant signal-to-noise ratio (SNR) during propagation.

Many efforts have been made in order to describe and quantify the speed of information in a dispersive medium and to investigate its compliance with the fundamental requirements of Einstein causality (which prohibits useful information to propagate with a velocity more than $c$ ). This topic will be thoroughly investigated in this thesis for the case of superluminal propagation.

\subsection{Connection between Velocity and Delay}

The previous section introduced five different velocity terms that are typically used to characterize the propagation of an electromagnetic pulse in a dispersive medium. In this section the more universal concept of delay is discussed. Characterizing propagation in terms of delay instead of velocity is more comprehensive as it applies to both the case of spatially negligible media (i.e. lumped circuits and elements at RF and microwave frequencies) and spatially extended media (i.e. optical frequencies).

\subsubsection{Phase Delay}

For propagation in a medium with length $L$, the phase delay $\left(\tau_{p}\right)$ is related to the phase velocity $v_{p}$ in a straight forward manner, where $v_{p}=L / \tau_{p}$. More generally, $\tau_{p}$ is expressed in terms of the phase response of the medium as

$$
\tau_{p}=\frac{-\phi(\omega)}{\omega} .
$$

Where, $\phi(\omega)=-k(\omega) L$. 


\subsubsection{Group Delay}

Group delay $\tau_{g}$ is associated with the time taken by the pulse envelope to travel a distance $L$ in a medium. As such, $\tau_{g}$ is related to the group velocity $v_{g}$ where, $v_{g}=L / \tau_{g}$. In a more formal way, $\tau_{g}$ can be expressed in terms of the phase response of the medium as

$$
\tau_{g}=\frac{-d \phi(\omega)}{d \omega}
$$

From Eq. 2.8, depending on the phase response of the medium, the group delay can either be positive or negative (in which case the output peak of the pulse is detected before the input peak interacts with the medium).

\subsection{Representation of Dispersion Terms}

In this section, the expressions needed to characterize the dispersion behavior in an arbitrary medium are introduced. Those expressions are written in terms of the aforementioned velocity and delay terms.

We start by introducing the dispersion expressions in terms of the phase and group velocity definitions. For a dispersive medium, the wave number $(k(\omega))$ is expressed as $k(\omega)=\frac{\omega n(\omega)}{c}$. In fact, using a second order Taylor expansion about the carrier frequency $\omega_{c}$, the term $k(\omega)$ can be expressed as

$$
k(\omega)=k\left(\omega_{c}\right)+\left.\frac{d k}{d \omega}\right|_{\omega_{c}}\left(\omega-\omega_{c}\right)+\left.\frac{1}{2} \frac{d^{2} k}{d \omega^{2}}\right|_{\omega_{c}}\left(\omega-\omega_{c}\right)^{2}+\ldots
$$

Equivalently, substituting Eq. 2.1 and Eq.2.3 in Eq. 2.9, $k(\omega)$ can be written in terms of $v_{p}$ and $v_{g}$ as follows:

$$
k(\omega)=\frac{\omega_{c}}{v_{p}}+\frac{1}{v_{g}}\left(\omega-\omega_{c}\right)+\frac{-1}{2 v_{g}^{2}} \frac{d v_{g}}{d \omega}\left(\omega-\omega_{c}\right)^{2}+\ldots
$$

Where, $\frac{d^{2} k}{d \omega^{2}}=\frac{-1}{v_{g}^{2}} \frac{d v_{g}}{d \omega}$ signifies the group velocity dispersion (GVD) which is responsible for pulse compression or broadening that is typically accompanied with pulse propagation in a dispersive medium. 
Likewise, dispersion can be expressed in terms of the phase and group delays. For a temporally dispersive medium, the phase associated with propagation for a distance $L$ is given as $\phi(\omega)=-k(\omega) L$. Expanding $\phi(\omega)$ via Taylor expansion about the carrier frequency $\omega_{c}$ yields the following expression:

$$
\phi(\omega)=\phi\left(\omega_{c}\right)+\left.\frac{d \phi}{d \omega}\right|_{\omega_{c}}\left(\omega-\omega_{c}\right)+\left.\frac{1}{2} \frac{d^{2} \phi}{d \omega^{2}}\right|_{\omega_{c}}\left(\omega-\omega_{c}\right)+\ldots
$$

Alternatively, from Eq. 2.7 and Eq. 2.8, the term $\phi(\omega)$ can be expressed in terms of $\tau_{p}$ and $\tau_{g}$ as follows:

$$
\phi(\omega)=-\left[\tau_{p} \omega_{c}+\tau_{g}\left(\omega-\omega_{c}\right)+\frac{1}{2} G D D\left(\omega-\omega_{c}\right)^{2}\right]+\ldots
$$

Where the term $G D D=\frac{-1}{2} \frac{d^{2} \phi}{d \omega^{2}}$ accounts for the group delay dispersion.

In this chapter, the basic definitions for velocities and delays encountered in a dispersive medium have been presented. It has been shown that these definitions are closely interrelated. Additionally, the general description of dispersive media has been presented in terms of these velocity and delay definitions. These concepts are used throughout the thesis in the subsequent chapters. In the next chapter we introduce causal dispersive media that follow a Lorentzian description. Regions in which superluminal (or subluminal) propagation takes place are highlighted and the methodology for calculating the corresponding field response is presented. 


\section{Chapter 3}

\section{Method of Steepest Descent in Causal Dielectrics}

In this chapter we introduce a causal dispersive media with an index of refraction $(n(\omega))$ that follows a Lorentzian model. In order to calculate the field response in such medium, the method of steepest descent is utilized. This method is regarded as a powerful tool that describes the time-frequency dynamics of the pulse and will be used throughout the thesis. As such, this method is first introduced here and applied to calculate the field response in a passive single resonance Lorentzian medium (that is well known in the literature); and the results are verified with the finite-difference time-domain analysis. The analysis is then extended to double resonance Lorentzian media with gain in the subsequent chapters.

\subsection{Lorentzian Media}

Throughout this analysis, a temporally dispersive medium that follows a Lorentzian model description is considered. In general, the index of refraction for such a medium is given by

$$
n(\omega)=\sqrt{1 \pm \frac{\omega_{p, 1}^{2}}{\omega^{2}-\omega_{0,1}^{2}+2 i \delta \omega} \ldots \pm \frac{\omega_{p, n}^{2}}{\omega^{2}-\omega_{0, n}^{2}+2 i \delta \omega}} .
$$

Where $\omega_{p}$ denotes the plasma frequency (or the dispersion strength) and is expressed in the form $\omega_{p}=\sqrt{\frac{N e^{2}}{m \epsilon_{0}}}$. 
Here, $N$ is the number density of the valence electrons, $m$ is the electron mass and $e$ is the electric charge. The term $\omega_{0}$ denotes the resonance frequency and $\delta$ is the phenomenological line-width. The subscript $n$ determines the number of resonances and the \pm signs denotes whether the resonance is associated with gain (+ve sign) or absorption (-ve sign), respectively.

In the following analysis, a single resonance Lorentzian medium with absorption is considered. The frequency domain behavior of the medium absorption and group delay is presented and the respective output field response is calculated using the method of steepest descent and verified using FDTD. Such analysis can then be extended to cover the case of double resonance gain media which are considered in the following chapters.

A passive Lorentzian medium with single resonance can be represented as [47]

$$
n(\omega)=\sqrt{1-\frac{\omega_{p}^{2}}{\omega^{2}-\omega_{0}^{2}+2 i \delta \omega}} .
$$

The numerical values for the Lorentzian medium parameters are given in [47] and are listed in Table 3.1 for convenience.

Table 3.1: Numerical values for the parameters of the passive single resonance Lorentzian medium.

\begin{tabular}{|c|c|}
\hline$\omega_{0}$ & $4 \times 10^{16}\left(\mathrm{sec}^{-1}\right)$ \\
\hline$\omega_{p}$ & $4.4721 \times 10^{16}\left(\mathrm{sec}^{-1}\right)$ \\
\hline$\delta$ & $0.28 \times 10^{16}\left(\mathrm{sec}^{-1}\right)$ \\
\hline
\end{tabular}

For a single resonance Lorentzian medium with the parameters listed in Table 3.1 . the real and imaginary parts of $n(\omega)$ are plotted in Fig. 3.1. Apparently, the index of refraction is no longer constant as in the case of a vacuum channel.

As introduced in Chapter 2, the group delay is given as $\tau_{g}=\frac{-d \phi(\omega)}{d \omega}$. In this case, $\phi(\omega)$ is the phase associated with the term $e^{-i \omega R e\{n(\omega)\} L / c}$; where, $L$ is the propagation distance and $c$ is the speed of light in vacuum. Likewise, the medium loss (or gain) factor is expressed as $e^{-\omega \Im[n(\omega)] L / c}$. The frequency domain behavior of the group delay and absorption for such a medium is plotted in Fig. 3.2 at a propagation distance of 0.25 $\mu m$. Clearly, there is a region of anomalous dispersion around the resonance frequency 


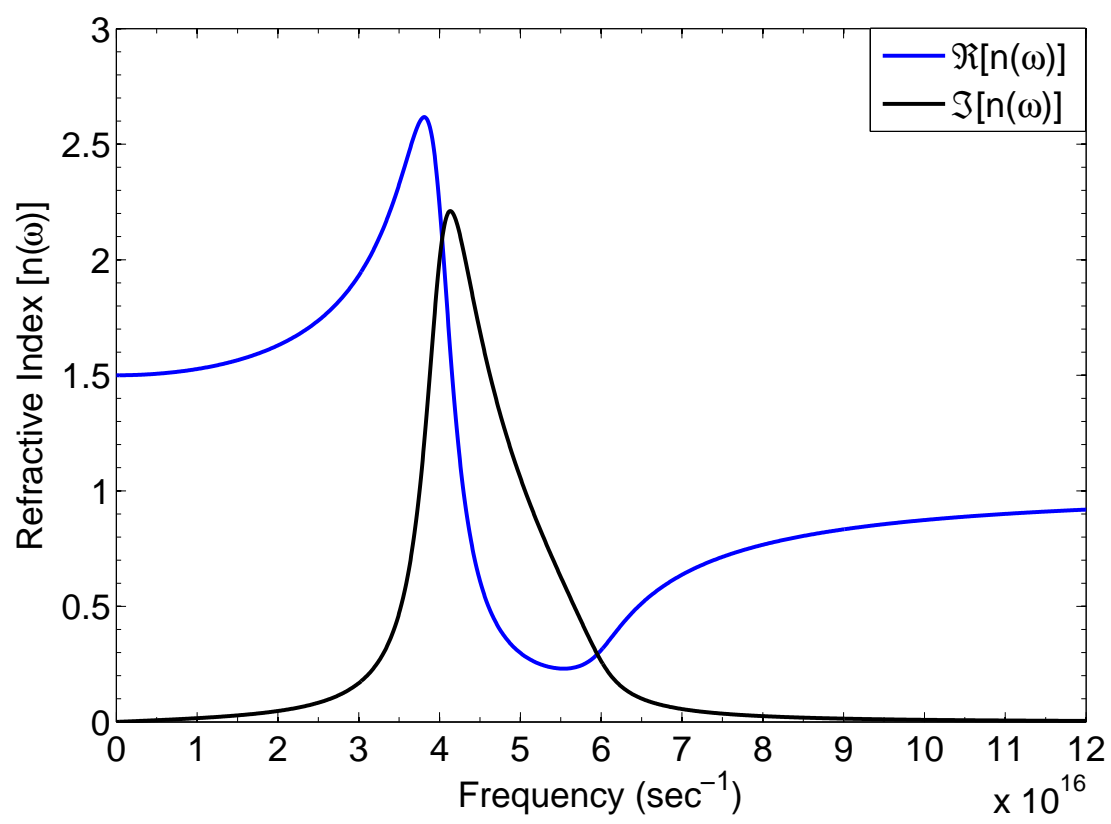

Figure 3.1: Real and imaginary parts of $n(\omega)$ as a function of frequency. Clearly, there exists a region of anomalous dispersion around the resonance frequency $\omega_{0}=4 \times 10^{16}\left(\mathrm{sec}^{-1}\right)$.

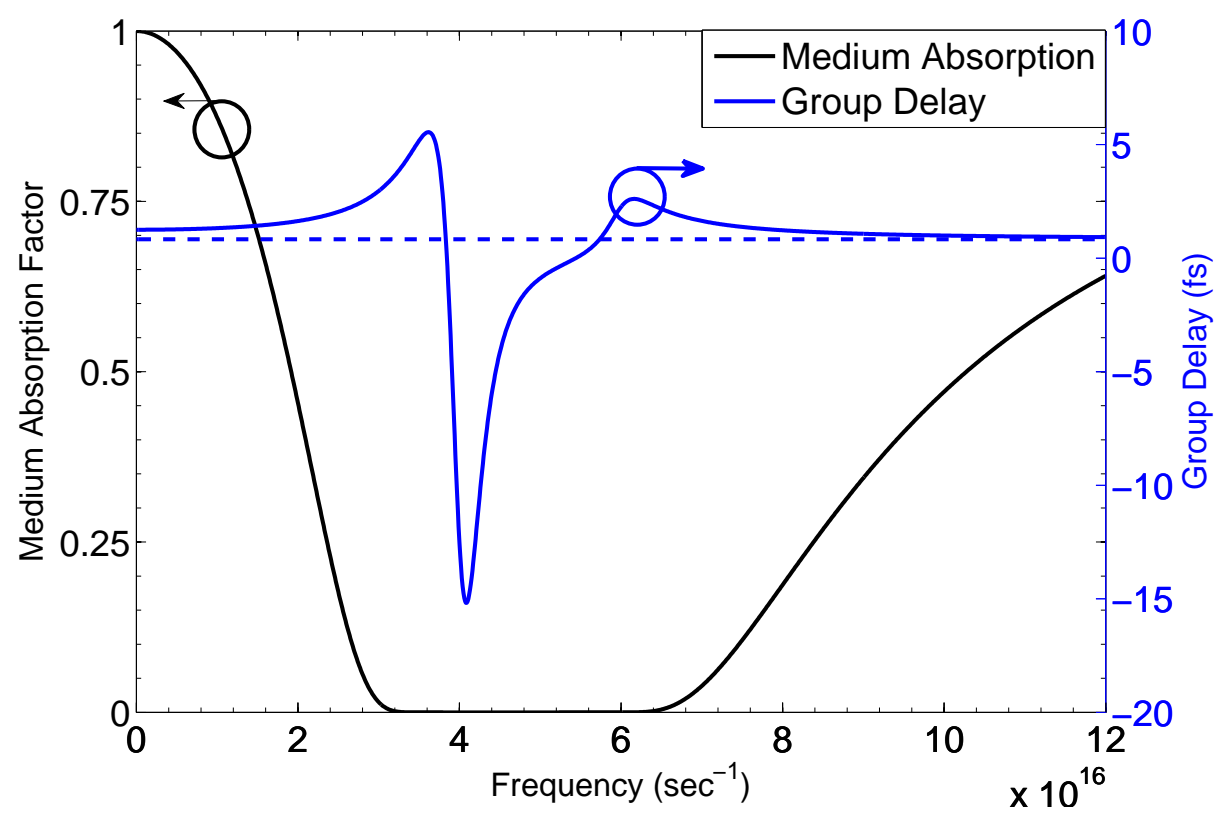

Figure 3.2: Frequency domain behavior of the passive Lorentzian medium. The medium absorption and group delay are calculated for a propagation distance equal to $0.25(\mu \mathrm{m})$. The blue dashed line corresponds to the luminal group delay in a vacuum channel of the same length.

$\omega_{0}=4 \times 10^{16}\left(\mathrm{sec}^{-1}\right)$. This region corresponds to negative group delay over a finite range of frequencies. Moreover, it can be inferred from the absorption behavior that the lower 
frequency components are less attenuated with respect to the high frequency components above resonance.

In this section, a brief overview of Lorenztian media has been presented. In the next section, the method of steepest descent (saddle point analysis) is introduced in order to calculate the field response propagating in such a medium. Since this analysis is a prerequisite to the field calculations in the subsequent chapters as well, the method is also verified using the finite-difference time-domain (FDTD) calculations.

\subsection{Method of Steepest Descent}

The reshaping phenomena of an electromagnetic pulse together with the classical theory of optical signal propagation in a homogeneous, isotropic, causally dispersive medium was first considered by Sommerfeld [1] and Brillouin[2, 3] in 1914. In their analysis, they investigated the propagation of an instantaneous turn-on signal with monochromatic carrier frequency in a single resonance Lorentz medium. Ougshtun and coworkers further improved the quantitative and qualitative analysis of the same problem using the steepest descent method (saddle point method). Saddle point method provides a detailed analysis of the time-frequency behavior of the propagating field. Such method has been applied to study the response of a single resonance [48, 49] and double resonance passive Lorentzian media to a modulated unit step excitation [50]. Afterwards, the behavior of an optical ultra-short Gaussian pulse has also been investigated, using the same method, at different propagation depths [51]. Later on, a generalized method for evaluating a Gaussian field with an arbitrary pulse width was introduced in [47]; as the method presented in [51] fails to accurately calculate the response of wide pulses with a temporal width that exceeds the characteristic relaxation time of a single resonance Lorentzian medium. More recently, the saddle point analysis has been extended to the case of a single resonance active Lorentzian media by considering a modulated unit step input [52] and a Gaussian excitation as well [53].

In this section, the method of steepest descent is introduced and applied to the case of a single resonance passive Lorentzian medium with the parameters listed in Table 3.1. The output response of a Gaussian excitation is then verified using the FDTD method. 
In order to calculate the output response for an input modulated Gaussian, we incorporate the generalized saddle point analysis in [47]. For the input excitation, we consider a causal Gaussian pulse with a pulse width $2 T=0.1(f s)$ and centered at a carrier frequency $\omega_{c}=5.75 \times 10^{16}\left(\mathrm{sec}^{-1}\right)$, which is the same configuration considered in [47]. The input pulse is temporally described in the following form:

$$
f(t)=e^{-\left(\frac{t-t_{0}}{T}\right)^{2}} \sin \left(\omega_{c} t\right)
$$

The pulse is excited at the plane $z=0$ and is centered at $t_{0} \sim 3 T$. The initial spectrum of $f(t)$ is given by

$$
F(\omega)=\sqrt{\pi} T e^{\frac{-T^{2}\left(\omega-\omega_{c}\right)^{2}}{4}} e^{-i \omega_{c} t_{0}}
$$

After propagating a distance $L$, the general description of the field, $E(z, t)$, is obtained from the integral:

$$
E(z, t)=\frac{1}{2 \pi} \operatorname{Re}\left[i \int \tilde{F} e^{z \phi\left(\omega, \theta^{\prime}\right) / c} d \omega\right]
$$

The term $\tilde{F}$ describes the spectral amplitude and is expressed as:

$$
\tilde{F}=\sqrt{\pi} T e^{-i \omega_{c} t_{0}}
$$

The phase term $\phi\left(\omega, \theta^{\prime}\right)$ for the Gaussian pulse is written in the following form:

$$
\phi\left(\omega, \theta^{\prime}\right)=i \omega\left[n(\omega)-\theta^{\prime}\right]-\frac{c T^{2}}{4 z}\left(\omega-\omega_{c}\right)^{2}
$$

where, $\theta^{\prime}$ is the dimensionless space-time parameter, $\theta^{\prime}=c\left(t-t_{0}\right) / z$ which maps to different time instants (given a fixed length $z$ ) [47].

The integration contour is taken to be the real frequency axis or any other contour that is homotopic to this axis. As such, the output response of the medium is evaluated by adding the contributions of the saddle point frequencies $\left(\omega_{S P}\right)$ that satisfy $\frac{d \phi\left(\omega=\omega_{S P}, \theta^{\prime}\right)}{d \omega}=$ 0 . This is equivalent to deforming the integration contour along the path of steepest descent of $\phi\left(\omega, \theta^{\prime}\right)$. Accordingly, the saddle points $\omega_{S P}$ are obtained - for the case of a Gaussian excitation in a single resonance passive Lorentzian medium — by finding the roots of the following equation at each instant of $\theta^{\prime}$ 


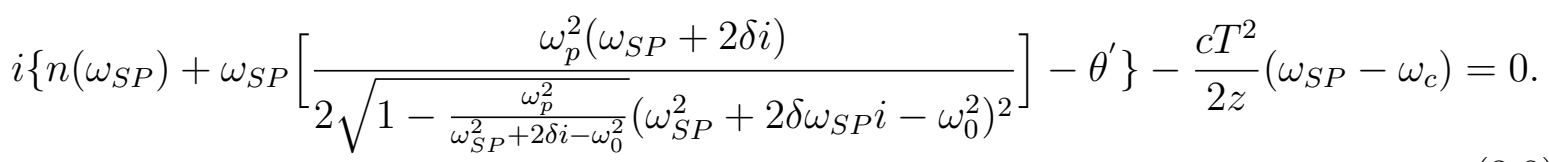

Equation 3.8 is numerically calculated at each instant of time that corresponds to the dimensionless parameters $\theta^{\prime}$. As such, the respective locations of the phase function $\phi\left(\omega, \theta^{\prime}\right)$ saddle points are plotted in Fig. 3.3. At each instant of $\theta^{\prime}$, there exists five different saddle points for $\phi\left(\omega, \theta^{\prime}\right)$, in full agreement with the analysis in [47]. The locations of these saddle points indicate the frequency components that contribute to the construction of the total output field response. Moreover, since the location of saddle points in the complex frequency domain are dynamic with respect to time, this gives insight to the instantaneous frequency oscillation of the field. Accordingly, pulse reshaping, distortion and chirping can be characterized by tracking the path of the saddle points in the complex frequency domain.

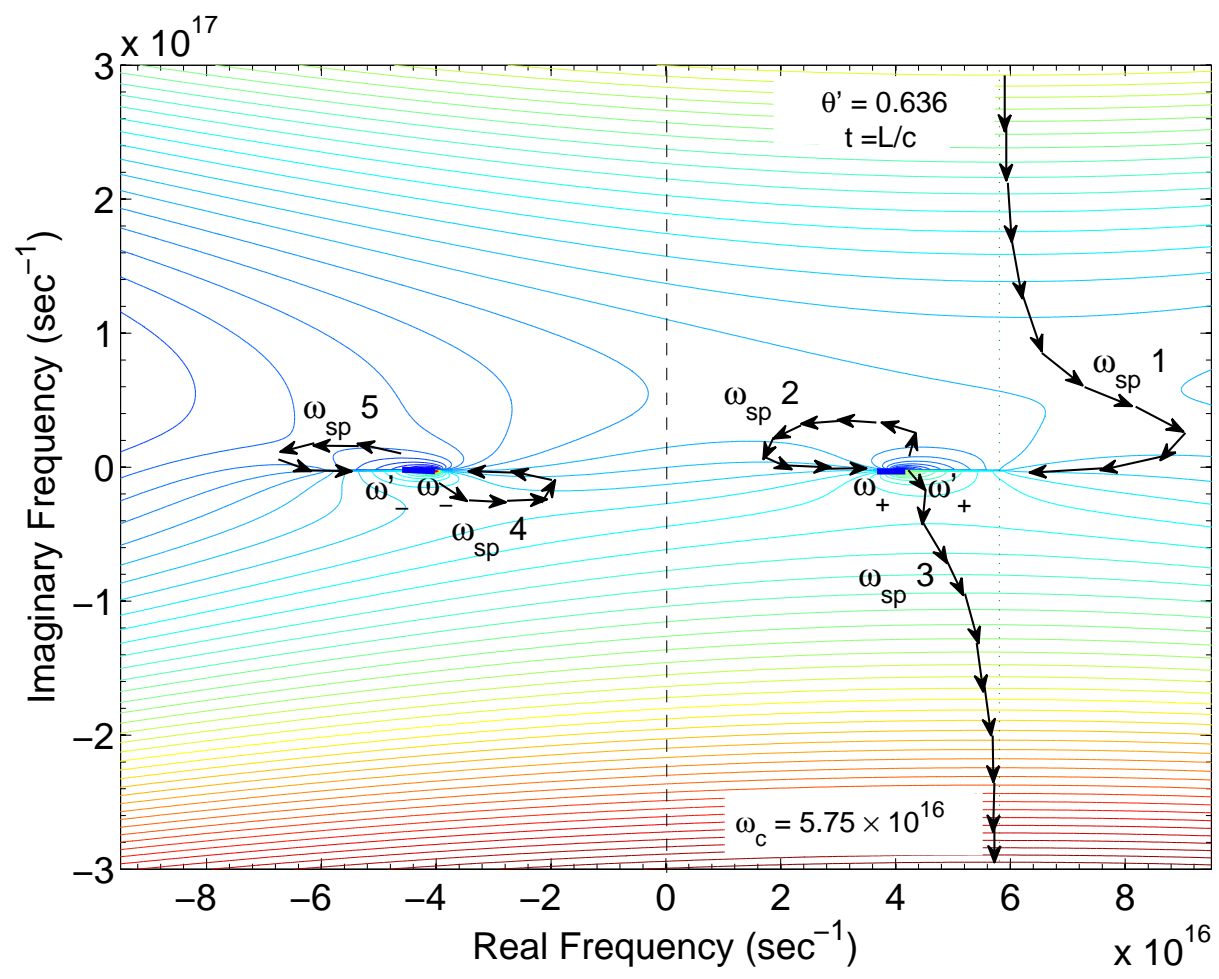

Figure 3.3: Path of the saddle points of $\phi\left(\omega, \theta^{\prime}\right)$ as a function of $\theta^{\prime}$ at a distance $L=0.25(\mu m)$ inside the Lorentzian medium. The colored contours illustrates the $\operatorname{Re}\left[\phi\left(\omega, \theta^{\prime}\right)\right]$ evaluated at $\theta^{\prime}=0.636$ (which corresponds to time $t=L / c)$. $\left(\omega_{+}, \omega_{+}^{\prime}\right)$ and $\left(\omega_{-}, \omega_{-}^{\prime}\right)$ signify the branch cuts (poles and zeroes) of the medium. 
In order to map the frequency behavior of the saddle points to the temporal value of the field at each instant of time $\left(\theta^{\prime}\right)$, the contribution of each saddle point frequency is evaluated by substituting $\omega_{S P}$ in the following expression [47]:

$$
A_{\omega_{S P}}\left(\theta^{\prime}\right)=\sqrt{\frac{c}{2 \pi z}} \operatorname{Re}\left[\frac{i \tilde{F} e^{\frac{z}{c} \phi\left(\omega_{S P}, \theta^{\prime}\right)}}{\sqrt{\frac{-d^{2}}{d \omega^{2}} \phi\left(\omega_{S P}, \theta^{\prime}\right)}}\right] .
$$

Where, $A_{\omega_{S P}}\left(\theta^{\prime}\right)$ is the contribution of each individual saddle point $\omega_{S P}$ at a given instant of time $\theta^{\prime}$ and the term $\frac{d^{2}}{d \omega^{2}} \phi\left(\omega_{S P}\right)$ is given by

$$
\frac{d^{2}}{d \omega^{2}} \phi\left(\omega_{S P}\right)=i\left[\omega \frac{d^{2}}{d \omega^{2}} n(\omega)+2 \frac{d n(\omega)}{d \omega}\right]-\frac{c T^{2}}{2 z},
$$

For a single resonance passive Lorentzian medium such as the one defined in Eq. 3.2, we obtain the following expressions for $\frac{d n(\omega)}{d \omega}$ and $\frac{d^{2}}{d \omega^{2}} n(\omega)$ :

$$
\frac{d n(\omega)}{d \omega}=\frac{\omega_{p}^{2}(2 \omega+2 \delta i)}{2 \sqrt{1-\frac{\omega_{p}^{2}}{\omega^{2}+2 \delta i-\omega_{0}^{2}}}\left(\omega^{2}+2 \delta \omega i-\omega_{0}^{2}\right)^{2}},
$$

and

$$
\begin{array}{r}
\frac{d^{2}}{d \omega^{2}} n(\omega)=\frac{\omega_{p}^{2}}{\sqrt{1-\frac{\omega_{p}^{2}}{\omega^{2}+2 \delta i-\omega_{0}^{2}}}\left(\omega^{2}+2 \delta \omega i-\omega_{0}^{2}\right)^{2}}-\frac{\omega_{p}^{2}(2 \omega+2 \delta i)^{2}}{\sqrt{1-\frac{\omega_{p}^{2}}{\omega^{2}+2 \delta i-\omega_{0}^{2}}}\left(\omega^{2}+2 \delta \omega i-\omega_{0}^{2}\right)^{3}} \\
-\frac{\omega_{p}^{2}(2 \omega+2 \delta i)^{2}}{4 \sqrt{\left(1-\frac{\omega_{p}^{2}}{\omega^{2}+2 \delta i-\omega_{0}^{2}}\right)^{3}}\left(\omega^{2}+2 \delta \omega i-\omega_{0}^{2}\right)^{4}} .
\end{array}
$$

In order to calculate the total field response, the contributions of each of the saddle points evaluated from Eq. 3.9 are added together. Consequently, the asymptotic description of the total field is represented as:

$$
A_{\text {Total }}\left(\theta^{\prime}\right)=\sum_{i=1}^{n} A_{\omega_{S P i}}\left(\theta^{\prime}\right) .
$$

Using Eq. 3.13, the total field is calculated at four different propagation distances: $0.25,0.5,0.75$ and $1(\mu \mathrm{m})$, respectively. The output response is verified using the finite- 
difference time-domain method 11 and the comparison is depicted in Fig. 3.4

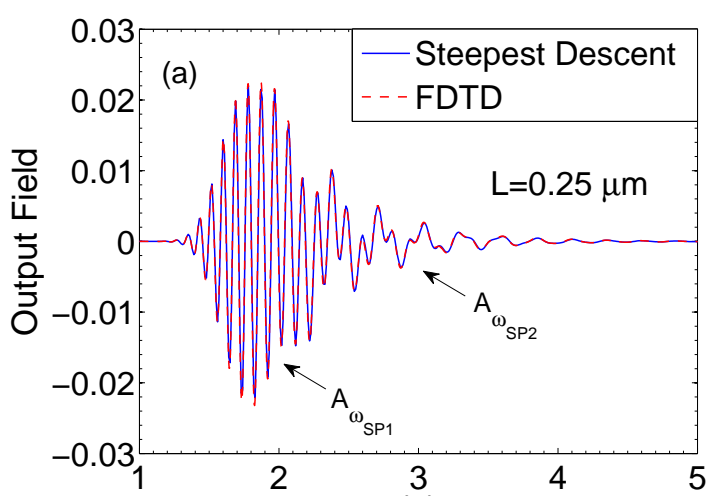

$(\theta)$

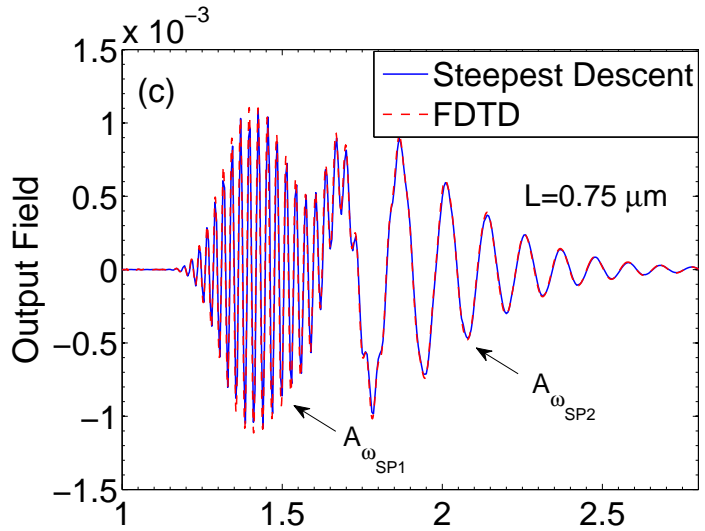

$(\theta)$
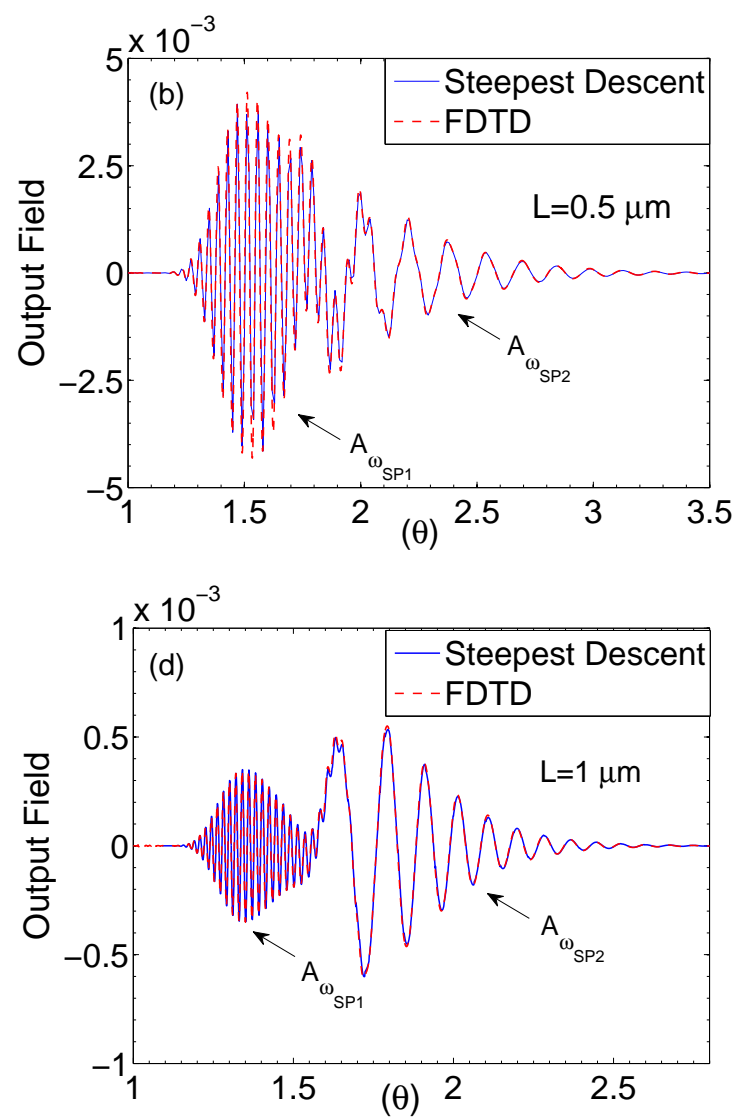

Figure 3.4: Time domain response of the propagating field as a function of the dimensionless space-time parameter $\theta(\theta=c t / z)$. Cases (a-d) corresponds to propagation distance $0.25,0.5,0.75$ and $1(\mu m)$, respectively.

From Fig. 3.4, it is clear that the time response of the saddle point method matches very well with the FDTD simulations. It can be observed from the figure that after propagation a long distances the pulse is decomposed into two main components; a high frequency component that lies above resonance and a delayed low frequency component. In fact, these two components are the dominant parts of the pulse and represent the contributions of the saddle points $\omega_{S P 1}$ and $\omega_{S P 2}$ that are illustrated in Fig. 3.3. It is observed that the high frequency components (which correspond to $\omega_{S P 1}$ ) are attenuated at a faster rate (in agreement with Fig. 3.2). At a propagation distance equal to $1(\mu m)$, the low frequency components (which correspond to $\omega_{S P 2}$ ) are less attenuated and dominate the propagating pulse.

\footnotetext{
${ }^{1}$ The detailed derivation of the update equations used in FDTD script is listed in Appendix A.
} 
Furthermore, the instantaneous frequency oscillation of the pulse is dynamic. As the propagation distance increases, the frequency components above resonance evolve to higher oscillations; this can be directly mapped to the frequency domain response of saddle point $\omega_{S P 1}$ which shifts to the right in the direction of higher frequencies as depicted in Fig. 3.3. On the other hand, the delayed low frequency components shift to lower frequency oscillations; this can also be directly mapped to the frequency domain response of the saddle point $\omega_{S P 2}$ which follows a circular path moving to the left in the direction of lower frequencies (in the same figure). As such, the method of steepest descent provides a detailed insight into the frequency domain dynamics of the pulse in addition to mapping these dynamics to the instantaneous temporal response of the field. In this chapter single resonance Lorentzian dispersive media have been introduced and the respective field calculations has been carried out using the method of steepest descent and verified using FDTD. The method of steepest descent will be extended in Chapter 4 to the case of an active double resonance Lorentzian medium in order to study the limitations imposed on superluminal propagation at longer propagation distances. Furthermore, the same method will be used in the subsequent chapter - chapter 5 - in order to investigate the possibility of superluminal signaling. 


\section{Chapter 4}

\section{Physical Dynamics in Superluminal Pulse Propagation}

Spectral reshaping and non-uniform phase delay associated with an electromagnetic pulse propagating in a temporally dispersive medium may lead to interesting observations in which the group velocity becomes superluminal or even negative. In such cases, the finite bandwidth of the superluminal region implies the inevitable existence of a cutoff distance beyond which a superluminal pulse becomes subluminal. In this chapter, we derive a closed form analytic expression to estimate this cutoff distance in abnormal dispersive media with gain. To the best of our knowledge, this is presented for the first time in literature. Moreover, the method of steepest descent is used to track the timefrequency dynamics associated with the evolution of the center-of-mass of a superluminal pulse to the subluminal regime. This evolution takes place at longer propagation depths (or narrower pulse width) as a result of the subluminal components affecting the behavior of the pulse. Finally, the analysis presents the fundamental limitations of superluminal propagation in light of factors such as the medium depth, pulse width and the medium dispersion strength.

\subsection{Introduction}

As discussed in Chapter 3, the spectral components comprising an electromagnetic pulse in a temporally dispersive medium are non-uniformly scaled and time shifted during 
propagation. In general, this leads to cases in which the pulse envelope may suffer from reshaping or distortion. Nevertheless, given the dispersion characteristics of a medium, one can design the wavelength and spectral width of the input pulse so that the interference among the spectral components leads to interesting observations in which the peak of the output pulse evolves at earlier time instants compared to its counterpart in vacuum (with minimum distortion); implying superluminal propagation. In other extreme cases, the pulse peak can even evolve at the output before the peak of the input pulse interacts with the medium; implying a negative group delay. Superluminal group delay and negative group delay refer to the same phenomenon, but from different frames-of-reference, and are collectively referred to as the abnormal group delay (AGD).

Many experiments at optical frequencies have demonstrated the possibility of achieving AGD using media with gain doublets [15, 16, 17, 18, 19]. In all such cases, it has been confirmed that the earliest response of the medium occurs after a strictly luminal duration equal to $(L / c)$ in compliance with the fundamental requirements of Einstein causality; where, $L$ is the medium length and $c$ is the speed of light in vacuum. In such experiments, the fact that the bandwidth of the AGD region is finite over a portion of the input spectrum implies that there always exists a crossover distance beyond which a superluminal pulse becomes subluminal, as predicted in [41]. This can be understood in the context of spectral leakage outside the superluminal region which is usually encountered by an electromagnetic pulse at longer propagation distances. To the best of our knowledge, there is no closed form expression in the literature by which $z_{\text {cutoff }}$ can be directly calculated. In previous efforts the cutoff distance was only calculated numerically [41] and, hence, the exact order dependency of $z_{\text {cutoff }}$ on parameters such as: the input pulse width and dispersion characteristics remained unexplored.

The goal of this chapter is: first, to derive a simple closed form analytic expression to predict the cutoff distance beyond which a superluminal pulse - propagating in a dispersive medium with gain - becomes subluminal. The derivation takes into account the input pulse width and the dispersive medium characteristics. The obtained expression quantifies the dependence of the cutoff distance on the characteristics of both the input pulse and the medium. This is useful for understanding the capabilities and setting the design constraints of a broad class of systems that utilize superluminal propagation. 
Second, we illustrate the time-frequency dynamics associated with the evolution of a superluminal pulse to the subluminal regime at longer propagation distance. Using the saddle point analysis (method of steepest descent), a superluminal pulse is decomposed into its superluminal and subluminal components. By tracking the evolution of these components under different configurations (different medium lengths), the physical mechanisms for the transition of a superluminal pulse to a subluminal are explained in light of the derived closed form expression. It is shown that this transition takes place when the subluminal components dominate over the pulse. Furthermore, we point out to the duality between the transition from superluminal to subluminal propagation as a result of traveling for a longer distance, as compared to using wide-band pulses at shorter propagation depths.

This chapter is organized as follows: in section 4.2, we introduce the dispersive medium considered throughout this analysis and we present the closed form analytic expression that predicts the cutoff distance beyond which a superluminal pulse becomes subluminal. The accuracy of the derived expression is then tested versus the exact calculations. The fundamental limitations of superluminal propagation in light of factors such as: the medium depth, pulse width, and the medium dispersion strength are thus discussed. Afterwards, in section 4.3, we present a brief overview on the steepest descent method used to calculate the evolution of an electromagnetic pulse traveling in a temporally dispersive gain doublet. In section 4.4, we discuss the time-frequency evolution of an electromagnetic pulse as a result of varying the medium length and highlight the effects of the medium length on superluminal propagation. In section 4.5, we perform a similar analysis for different scenarios of the input pulse width.

\subsection{Double Resonance Lorentzian Media with Gain}

We consider a dispersive medium that exhibits AGD in the flat region between a gain doublet. Such medium can be realized using the gain line of ammonia vapour cells at the wavelength of Rb laser (780 nm). The index of refraction for such an inverted medium is described by a double resonance Lorentzian gain function as follows [21]: 


$$
n(\omega)=\sqrt{1+\frac{\omega_{p, 1}^{2}}{\omega^{2}-\omega_{0,1}^{2}+2 i \delta \omega}+\frac{\omega_{p, 2}^{2}}{\omega^{2}-\omega_{0,2}^{2}+2 i \delta \omega}} .
$$

Where, $\omega_{p, j}$ and $\omega_{0, j}(\mathrm{j}=1$ or 2$)$ are the plasma and resonance frequencies associated with the first and second resonances, and $\delta$ denotes the phenomenological line-width. The numerical values for the Lorentzian medium parameters are listed in Table 4.1 .

Table 4.1: Numerical values for the parameters of the double resonance Lorenztian medium with gain (ammonia vapor cells).

\begin{tabular}{|c|c|}
\hline$\omega_{0,1}$ & $2.4165825 \times 10^{15}(\mathrm{rad} / \mathrm{sec})$ \\
\hline$\omega_{0,2}$ & $2.4166175 \times 10^{15}(\mathrm{rad} / \mathrm{sec})$ \\
\hline$\omega_{p, 1}$ & $10 \times 10^{9}(\mathrm{rad} / \mathrm{sec})$ \\
\hline$\omega_{p, 2}$ & $10 \times 10^{9}(\mathrm{rad} / \mathrm{sec})$ \\
\hline$\delta$ & $2.5 \times 10^{9}(\mathrm{rad} / \mathrm{sec})$ \\
\hline
\end{tabular}

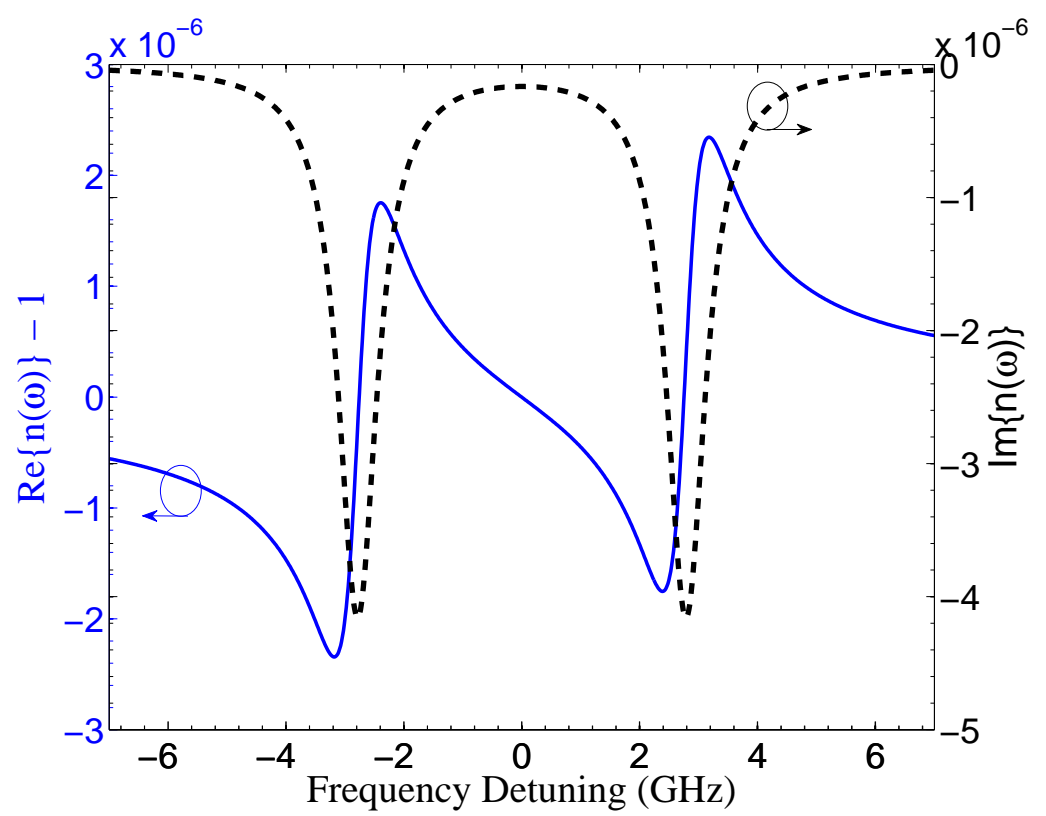

Figure 4.1: Index of refraction as a function of detuning, the left axis represents the real part and the right axis represents the imaginary part of $n(\omega)$, respectively.

For each of the resonances, the typical condition $\delta<\omega_{p, j}<\omega_{0, j}$ is satisfied. Such medium has a causal response and, consequently, the Kramers-Kronig relations are valid. The real and imaginary components of the index of refraction $n(\omega)$ are plotted in Fig 4.1 . 
The group delay of the medium $\left(\tau_{g}\right)$ with a depth $L$ is expressed as $\tau_{g}=\frac{-d \phi}{d \omega}$ where $\phi$ is the phase associated with the term $e^{-i \omega R e[n(\omega)] L / c}$. Figure 4.2 depicts the group delay at a propagation distance $25(\mathrm{~cm})$ inside the ammonia vapor cells. The separation between the medium resonances is in the order of $5 \mathrm{GHz}$; accordingly, we chose an input Gaussian pulse with spectrum that is initially contained within the superluminal region of the medium.

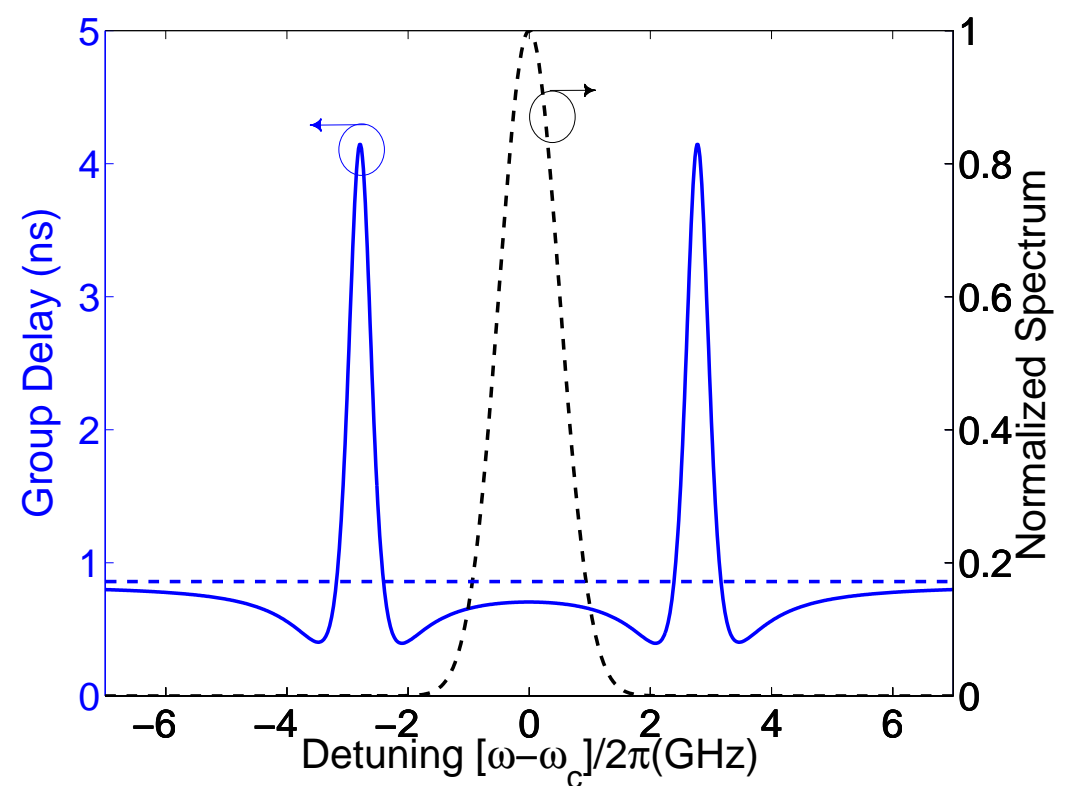

Figure 4.2: Group Delay $\tau_{g}$ as a function of frequency at a distance $25(\mathrm{~cm})$ plotted against the normalized initial pulse spectrum.

For the input excitation we consider a causal Gaussian pulse at $\lambda=780(\mathrm{~nm})\left(\omega_{c}=\right.$ $2.4166 \times 10^{15}(\mathrm{rad} / \mathrm{sec})$ with a pulse width $2 T=0.8(\mathrm{~ns})$. The input pulse is then given by

$$
f(t)=e^{-\left(\frac{t-t_{0}}{T}\right)^{2}} \sin \left(\omega_{c} t\right) .
$$

The pulse is excited at the $z=0$ plane and is centered at $t_{0} \sim 3 T$. The initial spectrum for $f(t)$ is written as

$$
F(\omega)=\sqrt{\pi} T e^{\frac{T^{2}\left(\omega-\omega_{c}\right)^{2}}{4}} e^{-i \omega_{c} t_{0}} .
$$

For such an input pulse - with an initial spectrum that is well fitted within the gain doublet - a superluminal effect (group velocity) can be observed over finite propagation distances. However, a superluminal-to-subluminal pulse transition can still take place 
if the pulse propagates far enough in the medium, or if the medium dispersion parameters are tuned [41]. The superluminal pulse transition to a subluminal regime can be attributed to the subluminal components that dominate over the pulse at loner propagation distance.

For instance, figure 4.3(a) depicts the input pulse spectrum over the medium gain at four different propagation distances. As the propagation distance increases, the overlap region between the (subluminal) gain doublet and the initial pulse spectrum expands (as marked by the black arrows). Accordingly, the pulse spectrum is no longer confined within the superluminal (flat) region of the medium and is dominated by the amplified subluminal components.

Likewise, for a fixed propagation distance, there exists a Gaussian pulse (centered at the superluminal region) with a cutoff spectral width beyond which the pulse becomes subluminal, as depicted in Fig. 4.3(b). In that case, the gain doublet is fixed and the pulse spectrum leaks outside the superluminal region. Consequently, the overlap region with the subluminal part is expanded and the center-of-mass of the pulse is delayed.

Additionally, it should be noted that the medium dispersion strength, denoted by the plasma frequency $\left(\omega_{p}\right)$, plays an important role that is analogous to the medium depth. For instance, increasing the oscillator strength, $\omega_{p}$, for both resonances (at a fixed medium depth) yields a behavior that is illustrated in Fig. 4.3(c). In analogy with the cases discussed in Fig. 4.3 (a) and (b), there exists a value for $\omega_{p}$ beyond which a superluminal pulse becomes dominated by its subluminal components as a consequence of the interaction with the gain doublet.

Therefore, the interplay between factors such as the pulse-width, propagation depth, and oscillator strength impose fundamental constraints on the propagation distance over which a superluminal effect can be observed. In this chapter, we show that for the broad class of dispersive media with gain (that follow a double resonance Lorentzian function), the interplay between the aforementioned factors can be governed (to a certain degree of accuracy) through the following approximate closed form expression ${ }^{1}$;

$$
z_{\text {cutoff }} \approx \frac{c \delta\left(\omega_{0,2}-\omega_{0,1}\right)^{2} T^{2}}{4 \omega_{p}^{2}} .
$$

\footnotetext{
${ }^{1}$ The derivation of this expression has been carried out with a significant help from Abhinav Ramakrishnan (abhinav.ramakrishnan@utoronto.ca) and is provided in details in Appendix B.
} 
Equation 4.4 governs the relation between the pulse width and the characteristics of the medium (in terms of the dispersion strength, linewidth and length), and their effect on superluminal propagation.
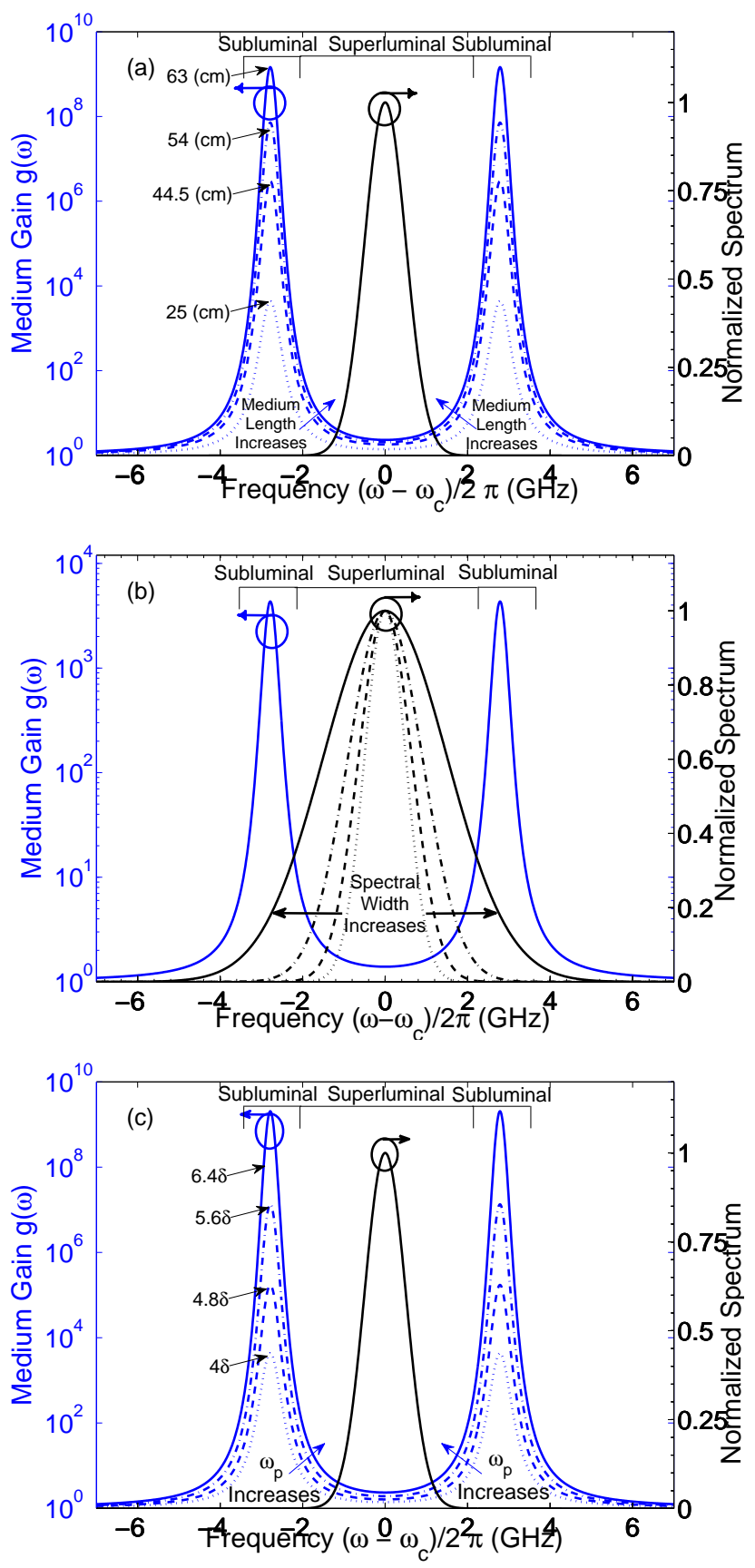

Figure 4.3: Spectral width and medium gain. (a) The medium gain $g(\omega)$ for length $L=25(\mathrm{~cm}), 44.5$ $(\mathrm{cm}), 54(\mathrm{~cm})$ and $63(\mathrm{~cm})$ and a Gaussian pulse with temporal width $2 T=0.9(\mathrm{~ns})$. (b) Medium gain $g(\omega)$ for a length $L=25(\mathrm{~cm})$ and pulse widths that correspond to $2 T=0.9(\mathrm{~ns}), 0.7(\mathrm{~ns}), 0.5(\mathrm{~ns})$ and $0.3(\mathrm{~ns})$, respectively. (c) The medium gain $g(\omega)$ at a fixed length $L=25(\mathrm{~cm})$ and pulse width $2 T$ $=0.9$ (ns) for oscillator strengths $\omega_{p}$ that is equal to $4 \delta, 4.8 \delta, 5.6 \delta$ and $6.4 \delta$, respectively 
In order to validate the accuracy of Eq4.4, the obtained cutoff distances are compared with the exact calculations over a wide range of parameters for the input pulse and the medium. The exact calculations of the cutoff distance are performed by evaluating the arrival time of the pulse at different values for the medium length, dispersion strength, and input pulse widths. The arrival time is calculated by a procedure that follows directly from Ref. [46]. This involves an averaging of the group delay weighted by the output pulse spectrum as described by

$$
\left\langle t_{f}\right\rangle=\frac{\int_{\omega_{1}}^{\omega_{2}} \tau_{g} g(\omega) \mathbf{F}(\omega) d \omega}{\int_{\omega_{1}}^{\omega_{2}} g(\omega) \mathbf{F}(\omega) d \omega},
$$

where $\tau_{g}$ is the group delay, $g(\omega)$ is the medium gain, and $F(\omega)$ is the input pulse spectrum obtained from Eq. 4.3 . The subscript $f$ in $\left\langle t_{f}\right\rangle$ denotes that the arrival time is calculated using a frequency domain analysis. By comparing the arrival time obtained from Eq. 4.5 with the strictly luminal delay $(L / c)$ over a wide range of propagation distances, one can then deduce the exact cutoff distance at which the superluminal-to-subluminal pulse transition occurs. The comparisons between the approximate expression and the exact calculations are depicted in Fig. 4.4

The cutoff distances predicted from Eq. 4.4 demonstrate a very good agreement with the exact calculations - with a maximum deviation of $5 \%$. In fact, it can be shown that the expression in Eq. 4.4 provides an asymptotic upper bound for the cutoff distance. Such expression is quite accurate as long as $\delta<\frac{\omega_{0,2}-\omega_{0,1}}{10}$ and $\left(\omega_{0,2}-\omega_{0,1}\right)<\frac{\omega_{0,1}}{10}$, which are typically the case in practice.

Equation 4.4 captures all the physical dynamics involved in the problem in a very compact form; it can be inferred that-for superluminal pulses with larger temporal width $(T)$ (narrow band pulses) - the transition to the subluminal region occurs at a longer cutoff distance $\left(z_{\text {cutoff }}\right)$ as depicted in Fig. 4.4(a). This also applies to a double resonance medium with wide frequency band between resonances (larger $\omega_{0,2}-\omega_{0,1}$ ) or larger linewidth $(\delta)$, in which cases the transition occurs at longer propagation distancesas shown in Fig. 4.4(b).

On the other hand, a superluminal pulse propagating in a medium with strong dis- 

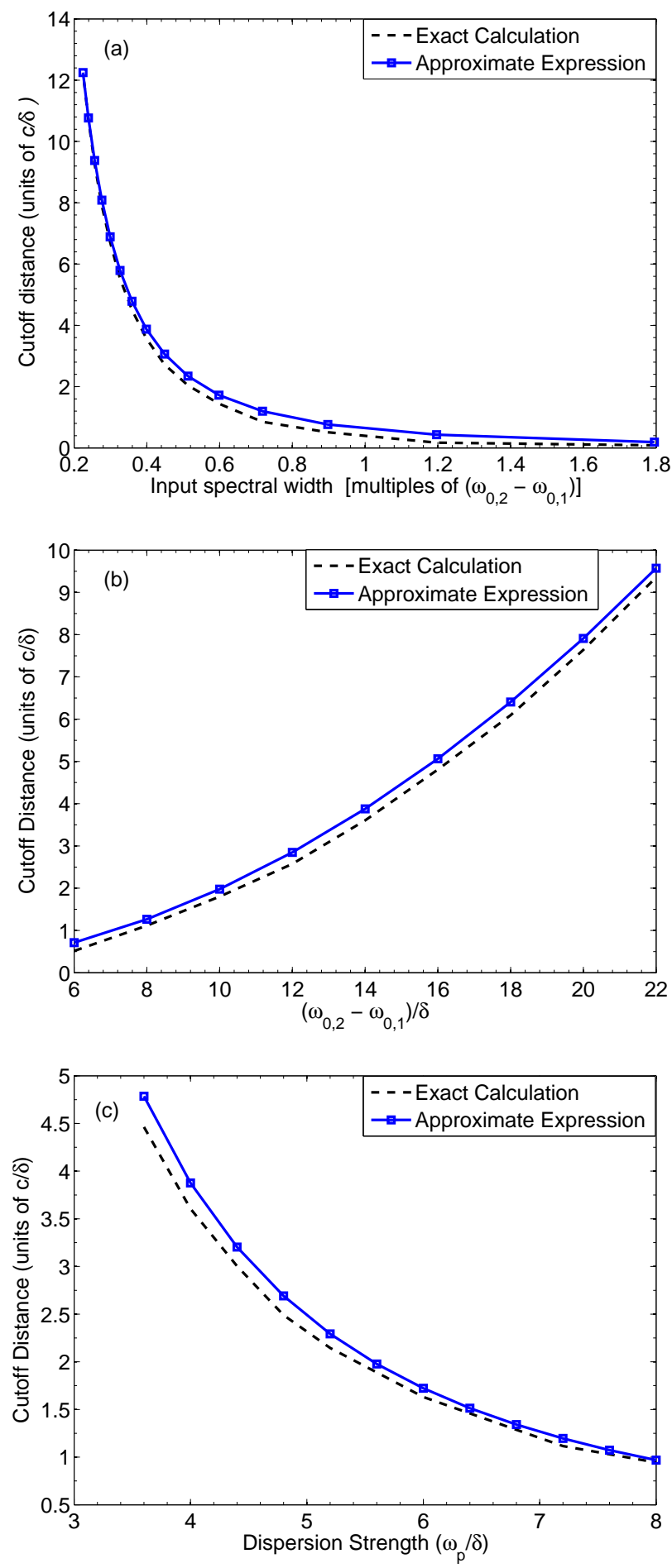

Figure 4.4: Comparison between the exact calculations of the cutoff distance versus the approximate closed form expression when: (a) The input spectral width is increased (or as the temporal pulse width is reduced). (b) The medium resonances $\left(\omega_{0,2}-\omega_{0,1}\right)$ are tuned. (c) The plasma frequency (dispersion strength) is increased. 
persion (larger value for $\omega_{p}$ ) would evolve to the subluminal regime at shorter cutoff

distance $\left(z_{\text {cutoff }}\right)$, as depicted in Fig. 4.4(c). Finally, it can be inferred from the higher order dependencies in Eq. 4.4, that the transition from superluminal to subluminal is more sensitive to the variations in the pulse width $(T)$, frequency detuning $\left(\omega_{0,2}-\omega_{0,1}\right)$, and dispersion strength $\left(\omega_{p}\right)$-all of which have a quadratic dependency - as compared to the variations in the medium length $(z)$ and the linewidth $(\delta)$.

In this section, we presented a closed form analytic expression for the cutoff distance at which superluminal-to-subluminal pulse transition occurs. Using this expression, this cutoff distance can be calculated without the need to know the exact spectral distribution of the input pulse. The knowledge of the initial pulse width $(T)$ is sufficient in such calculation. The expression has been verified by comparison with the exact frequency domain calculations. In the following sections, we calculate the temporal evolution of the field at different propagation distances to verify the approximate expression for the cutoff in the time domain. This also gives insight about the time-frequency dynamics that governs the superluminal-to-subluminal transition. In order to do so, the method of steepest descent is incorporated, as discussed next.

\subsection{Steepest Descent Analysis for Pulse propagation in a Gain medium}

After propagation for a distance $L$, the general description of the field $E(z, t)$ is obtained by solving the integral:

$$
E(z, t)=\frac{1}{2 \pi} \operatorname{Re}\left[i \int \tilde{F} e^{z \phi\left(\omega, \theta^{\prime}\right) / c} d \omega\right]
$$

The term $\tilde{F}$ describes the spectral amplitude and is expressed as

$$
\tilde{F}=\sqrt{\pi} T e^{-i \omega_{c} t_{0}}
$$

The phase term $\phi\left(\omega, \theta^{\prime}\right)$ for the Gaussian pulse is given by

$$
\phi\left(\omega, \theta^{\prime}\right)=i \omega\left[n(\omega)-\theta^{\prime}\right]-\frac{c T^{2}}{4 z}\left(\omega-\omega_{c}\right)^{2}
$$


where, $\theta^{\prime}$ is the dimensionless space-time parameter, $\theta^{\prime}=c\left(t-t_{0}\right) / z$ which maps to different time instants (given a fixed length $z$ ) [47]. The integration contour is carried over the real frequency axis or any other contour that is homotopic to this axis. As such, the output response of the medium is evaluated by adding the contributions of the saddle point frequencies $\left(\omega_{S P}\right)$ that satisfy $\frac{d \phi\left(\omega=\omega_{S P}, \theta^{\prime}\right)}{d \omega}=0$.

As such, the saddle points $\left(\omega_{S P}\right)$ are calculated by solving the following equation:

$$
\begin{array}{r}
i\left\{n(\omega)-\omega\left[\frac{\omega_{p}^{2}(2 \omega+2 \delta i)}{\left(\omega^{2}+2 \delta \omega i-\omega_{0}^{2}\right)^{2}}+\frac{\omega_{p, 2}^{2}(2 \omega+2 \delta i)}{\left(\omega^{2}+2 \delta \omega i-\omega_{0,2}^{2}\right)^{2}}\right] \frac{1}{\sqrt{\frac{2 \omega_{p}^{2}}{\omega^{2}+2 \delta i-\omega_{0}^{2}}+\frac{2 \omega_{p, 2}^{2}}{\omega^{2}+2 \delta i-\omega_{0,2}^{2}}+1}}-\theta^{\prime}\right\} \\
-\frac{c T^{2}}{2 z}\left(\omega-\omega_{c}\right)=0 .
\end{array}
$$

This is equivalent to deforming the integration contour along the path of steepest descent of $\phi\left(\omega, \theta^{\prime}\right)$. In order to obtain the value of the field at each instant of time $\left(\theta^{\prime}\right)$, the contribution of each saddle point frequency is evaluated by substituting the saddle points in the following expression [47]:

$$
A_{\omega_{S P}}\left(\theta^{\prime}\right)=\sqrt{\frac{c}{2 \pi z}} \operatorname{Re}\left[\frac{i \tilde{F} e^{\frac{z}{c} \phi\left(\omega_{S P}, \theta^{\prime}\right)}}{\sqrt{\frac{-d^{2}}{d \omega^{2}} \phi\left(\omega_{S P}, \theta^{\prime}\right)}}\right] .
$$

Where $A_{\omega_{S P}}\left(\theta^{\prime}\right)$ is the contribution of each individual saddle point $\omega_{S P}$ at a given instant of time $\theta^{\prime}$. The term $\frac{d^{2}}{d \omega^{2}} \phi\left(\omega_{S P}\right)$ is expressed as

$$
\frac{d^{2}}{d \omega^{2}} \phi\left(\omega_{S P}\right)=i\left[\omega \frac{d^{2}}{d \omega^{2}} n(\omega)+2 \frac{d n(\omega)}{d \omega}\right]-\frac{c T^{2}}{2 Z} .
$$

For an inverted-medium that is described by a double resonance Lorentzian, we obtain the following expressions for $\frac{d n(\omega)}{d \omega}$ and $\frac{d^{2}}{d \omega^{2}} n(\omega)$ :

$$
\frac{d n(\omega)}{d \omega}=\left[\frac{\omega_{p}^{2}(2 \omega+2 \delta i)}{\left(\omega^{2}+2 \delta \omega i-\omega_{0}^{2}\right)^{2}}+\frac{\omega_{p, 2}^{2}(2 \omega+2 \delta i)}{\left(\omega^{2}+2 \delta \omega i-\omega_{0,2}^{2}\right)^{2}}\right] \frac{1}{\sqrt{\frac{2 \omega_{p}^{2}}{\omega^{2}+2 \delta i-\omega_{0}^{2}}+\frac{2 \omega_{p, 2}^{2}}{\omega^{2}+2 \delta i-\omega_{0,2}^{2}}+1}}
$$


whereas,

$$
\begin{array}{r}
\frac{d^{2}}{d \omega^{2}} n(\omega)=-\left[\frac{\omega_{p}^{2}(2 \omega+2 \delta i)}{\left(\omega^{2}+2 \delta \omega i-\omega_{0}^{2}\right)^{2}}+\frac{\omega_{p, 2}^{2}(2 \omega+2 \delta i)}{\left(\omega^{2}+2 \delta \omega i-\omega_{0,2}^{2}\right)^{2}}\right] \frac{1}{4 \sqrt{\left(\frac{2 \omega_{p}^{2}}{\omega^{2}+2 \delta i-\omega_{0}^{2}}+\frac{2 \omega_{p, 2}^{2}}{\omega^{2}+2 \delta i-\omega_{0,2}^{2}}+1\right)^{3}}} \\
-\left[\frac{2 \omega_{p}^{2}}{\left(\omega^{2}+2 \delta \omega i-\omega_{0}^{2}\right)^{2}}+\frac{2 \omega_{p, 2}^{2}}{\left(\omega^{2}+2 \delta \omega i-\omega_{0,2}^{2}\right)^{2}}-\frac{2 \omega_{p}^{2}(2 \omega+2 \delta i)^{2}}{\left(\omega^{2}+2 \delta \omega i-\omega_{0}^{2}\right)^{3}}-\frac{2 \omega_{p, 2}^{2}(2 \omega+2 \delta i)^{2}}{\left(\omega^{2}+2 \delta \omega i-\omega_{0,2}^{2}\right)^{3}}\right] \\
\times \frac{1}{2 \sqrt{\left.\frac{2 \omega_{p}^{2}}{\omega^{2}+2 \delta i-\omega_{0}^{2}}+\frac{2 \omega_{p, 2}^{2}}{\omega^{2}+2 \delta i-\omega_{0,2}^{2}}+1\right)}} .
\end{array}
$$

In order to calculate the total field response for an electromagnetic pulse propagating in a temporally dispersive lorentzian medium as described in Eq 4.1, the contribution of each of the saddle points are added together. Accordingly, the asymptotic description of the total field is represented as:

$$
A_{\text {Total }}\left(\theta^{\prime}\right)=\sum_{i=1}^{n} A_{\omega_{S P, i}}\left(\theta^{\prime}\right) .
$$

As such, the method of steepest descent can be used to assess the dominance of the different spectral components of the pulse, part of which may be superluminal or subluminal, towards the construction of the total field. In the next section, we carry out this assessment for the different spectral components of pulse propagating in an AGD Lorentzian medium at different propagation distances to highlight the role played by the subluminal components in transforming a superluminal pulse to a subluminal regime and to verify the expression provided in Eq. 4.4 in the time domain as well.

\subsection{Physical Dynamics of a Superluminal Pulse at Longer Prop- agation Lengths}

In this section, we consider Gaussian pulse propagation in a dispersive medium with index of refraction that is given in $\mathrm{Eq} 4.1$. The behavior of the pulse is compared at four different propagation distances. As discussed in section 4.3, the total field is calculated by adding the contributions of $\omega_{S P}$ that satisfy $\frac{d \phi\left(\omega=\omega_{S P}, \theta^{\prime}\right)}{d \omega}=0$. The exact locations of 
the saddle points are numerically calculated at each instant of time $\left(\theta^{\prime}\right)$ for four different propagation distances: $25(\mathrm{~cm}), 44.5(\mathrm{~cm}), 54(\mathrm{~cm})$ and $63(\mathrm{~cm})$ and are plotted in Fig. 4.54 .64 .74 .8 , respectively.

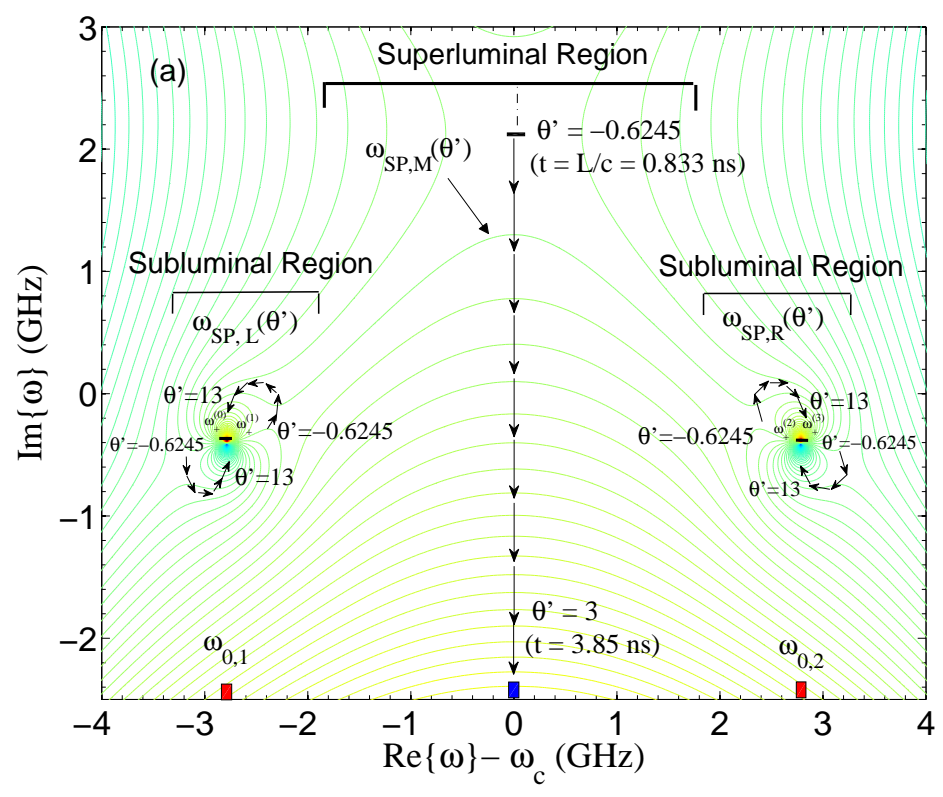

Figure 4.5: Path of the saddle points $\omega_{S P}$ of $\phi\left(\omega_{S P}, \theta^{\prime}\right)$ as $\theta^{\prime}$ increases at propagation distance $25(\mathrm{~cm})$ inside the ammonia vapor cells. The colored (green) contours show $\left[\operatorname{Re}\left\{\phi\left(\omega, \theta^{\prime}\right)\right\}\right]$ at $t=L / c$.

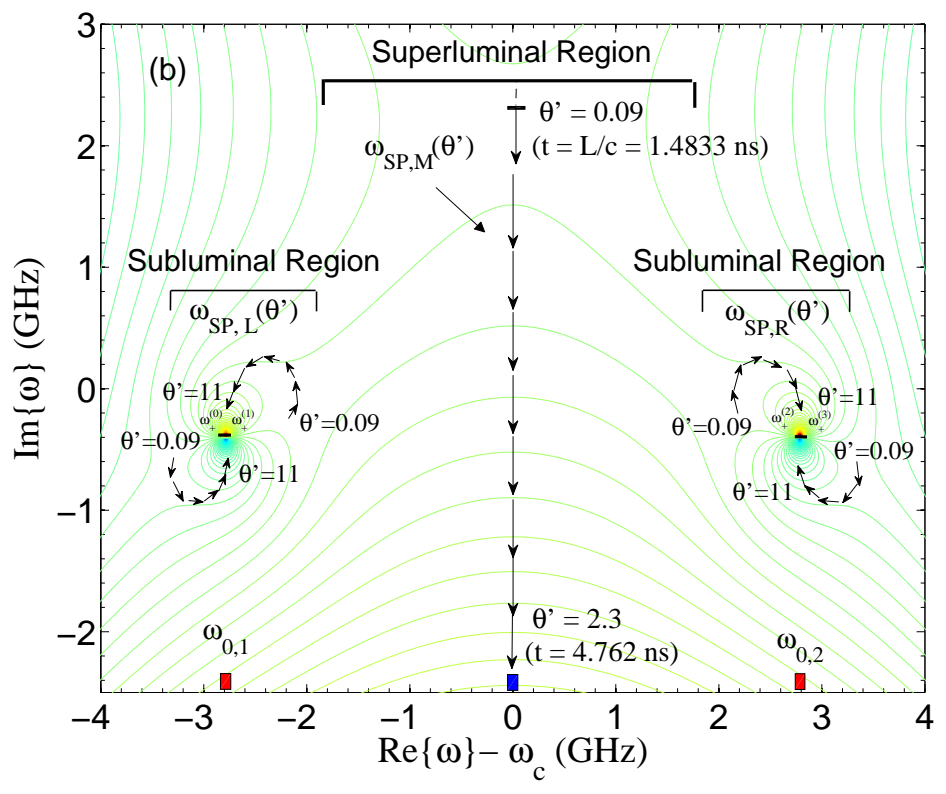

Figure 4.6: Path of the saddle points $\omega_{S P}$ of $\phi\left(\omega_{S P}, \theta^{\prime}\right)$ as $\theta^{\prime}$ increases at propagation distance $44.5(\mathrm{~cm})$ inside the ammonia vapor cells. The colored (green) contours show $\left[\operatorname{Re}\left\{\phi\left(\omega, \theta^{\prime}\right)\right\}\right]$ at $t=L / c$. 


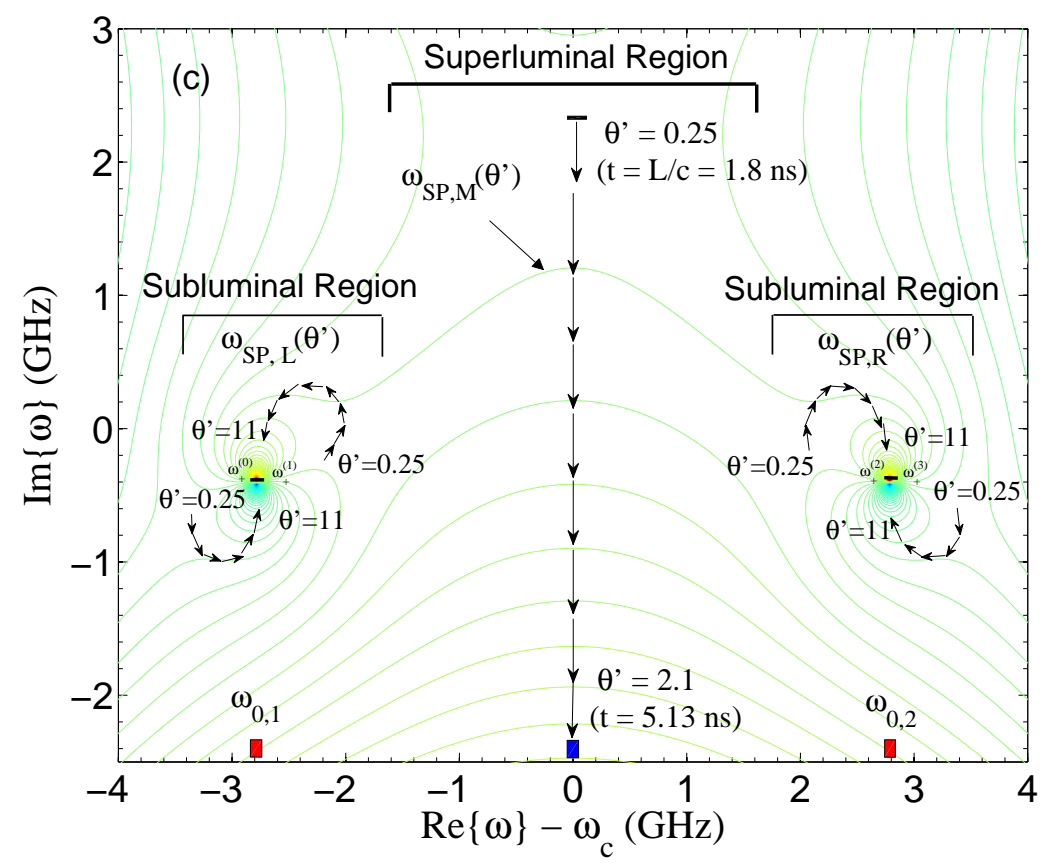

Figure 4.7: Path of the saddle points $\omega_{S P}$ of $\phi\left(\omega_{S P}, \theta^{\prime}\right)$ as $\theta^{\prime}$ increases at propagation distance $54(\mathrm{~cm})$ inside the ammonia vapor cells. The colored (green) contours show $\left[\operatorname{Re}\left\{\phi\left(\omega, \theta^{\prime}\right)\right\}\right]$ at $t=L / c$.

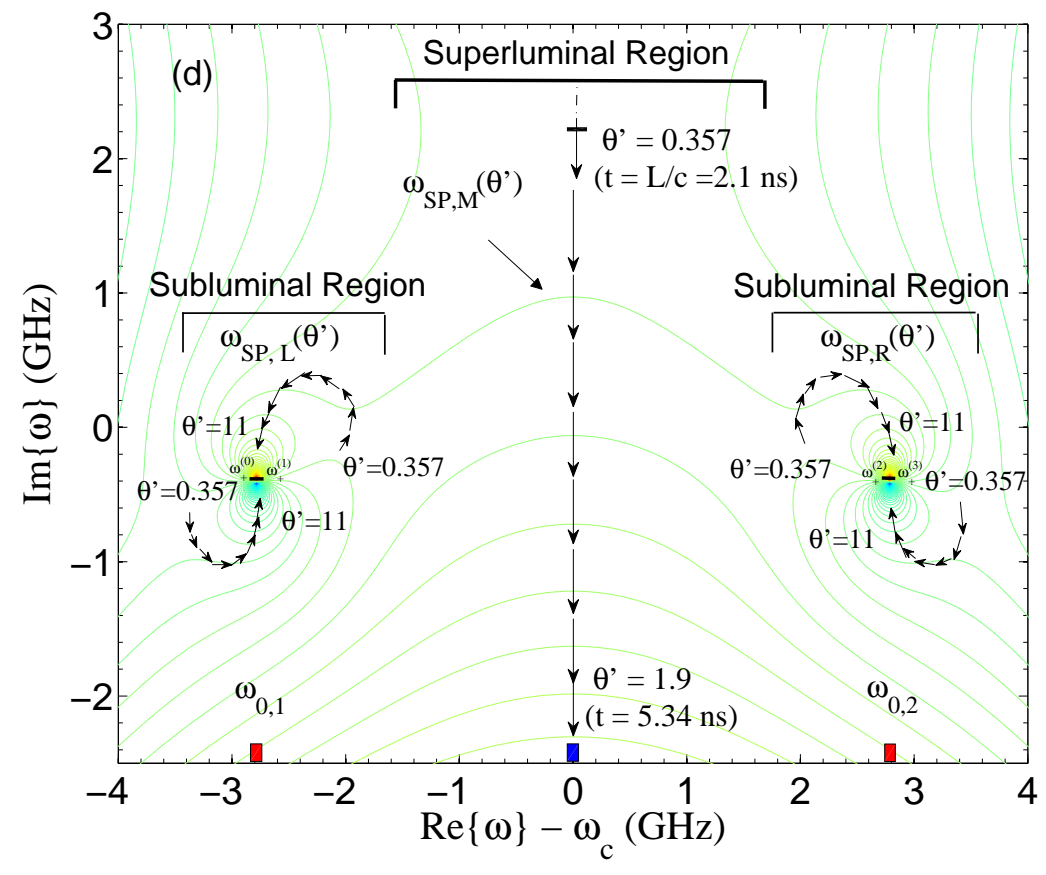

Figure 4.8: Path of the saddle points $\omega_{S P}$ of $\phi\left(\omega_{S P}, \theta^{\prime}\right)$ as $\theta^{\prime}$ increases at propagation distance $63(\mathrm{~cm})$ inside the ammonia vapor cells. The colored (green) contours show $\left[\operatorname{Re}\left\{\phi\left(\omega, \theta^{\prime}\right)\right\}\right]$ at $t=L / c$. 
In all figures 4.5 4.6, 4.7 4.8 , the colored contours describe the real part of the corresponding phase function $\left[\operatorname{Re} \phi\left(\omega, \theta^{\prime}\right)\right]$ at the respective value of $\left(\theta^{\prime}\right)$ which maps to the earliest response of the medium $(t=L / c)$. The first and second resonances of $n(\omega)$ are denoted by $\left(\omega_{0,1}\right)$ and $\left(\omega_{0,2}\right)$, respectively. The terms $\left(\omega_{+}{ }^{(0)}, \omega_{+}{ }^{(1)}\right)$ and $\left(\omega_{+}{ }^{(2)}, \omega_{+}{ }^{(3)}\right)$ signify the branch cuts for $n(\omega)$. The frequency ranges that correspond to the superluminal and the subluminal regions are shown in the same figure. The arrows depict the path of the dominant saddle points, which are labeled by $\omega_{S P, M}, \omega_{S P, L}$ and $\omega_{S P, R}$. Clearly, the saddle points paths exhibit a symmetry about the carrier frequency $\omega_{c}$. Moreover, it is found that at each instant of $\theta^{\prime}$, the contribution of the saddle point frequencies, $\omega_{S P, M}$, $\omega_{S P, L}$ and $\omega_{S P, R}$, are much more pronounced than the other two saddle points below the branch cuts. As such, the integration contour is deformed along the steepest descent path in the upper half complex plane of $\phi\left(\omega, \theta^{\prime}\right)$.

For a pulse with an initial spectrum well fitted within the superluminal region such as that depicted in Fig 4.5, it will be shown that the output field is dominated by the contribution of the middle saddle points $\omega_{S P, M}$ which follow a vertical path downwards at the carrier frequency in the middle of the superluminal group delay region. The other saddle points, $\omega_{S P, L}$ and $\omega_{S P, R}$, both of which lie in the subluminal frequency region, have minimal contributions to the construction of the total field. As the pulse further penetrates inside the medium, the interaction of the pulse side bands with the gain doublet becomes more pronounced. This is evident in the cases depicted in figures 4.6 through 4.8 in which the circular path of the saddle points $\omega_{S P, L}$ and $\omega_{S P, R}$ becomes more spread out around the branch cuts at longer propagation depths. As the radius of this circular path increases, approaching the carrier frequency $\omega_{c}$, the contributions of the corresponding (subluminal) saddle points become more significant.

The decomposed contributions of $\omega_{S P, M}$, denoted as $A_{\omega_{S P, M}}\left(\theta^{\prime}\right)$ and $\omega_{S P, L}$, denoted as $A_{\omega_{S P, L}}\left(\theta^{\prime}\right)$, are plotted in Fig 4.9 for the four propagation distances. The contributions of $\omega_{S P, R}$ maintain a close resemblance with the contributions of $\omega_{S P, L}$ contribution and are not included in the plots for the sake of brevity. As the propagation length increases from $25(\mathrm{~cm})$ to $63(\mathrm{~cm})$, the superluminal component, associated with $\omega_{S P, M}$ experiences slight amplification and compression. On the other hand, the subluminal components, associated with $\omega_{S P, L}$ and $\omega_{S P, R}$, experience broadening and are more significantly am- 

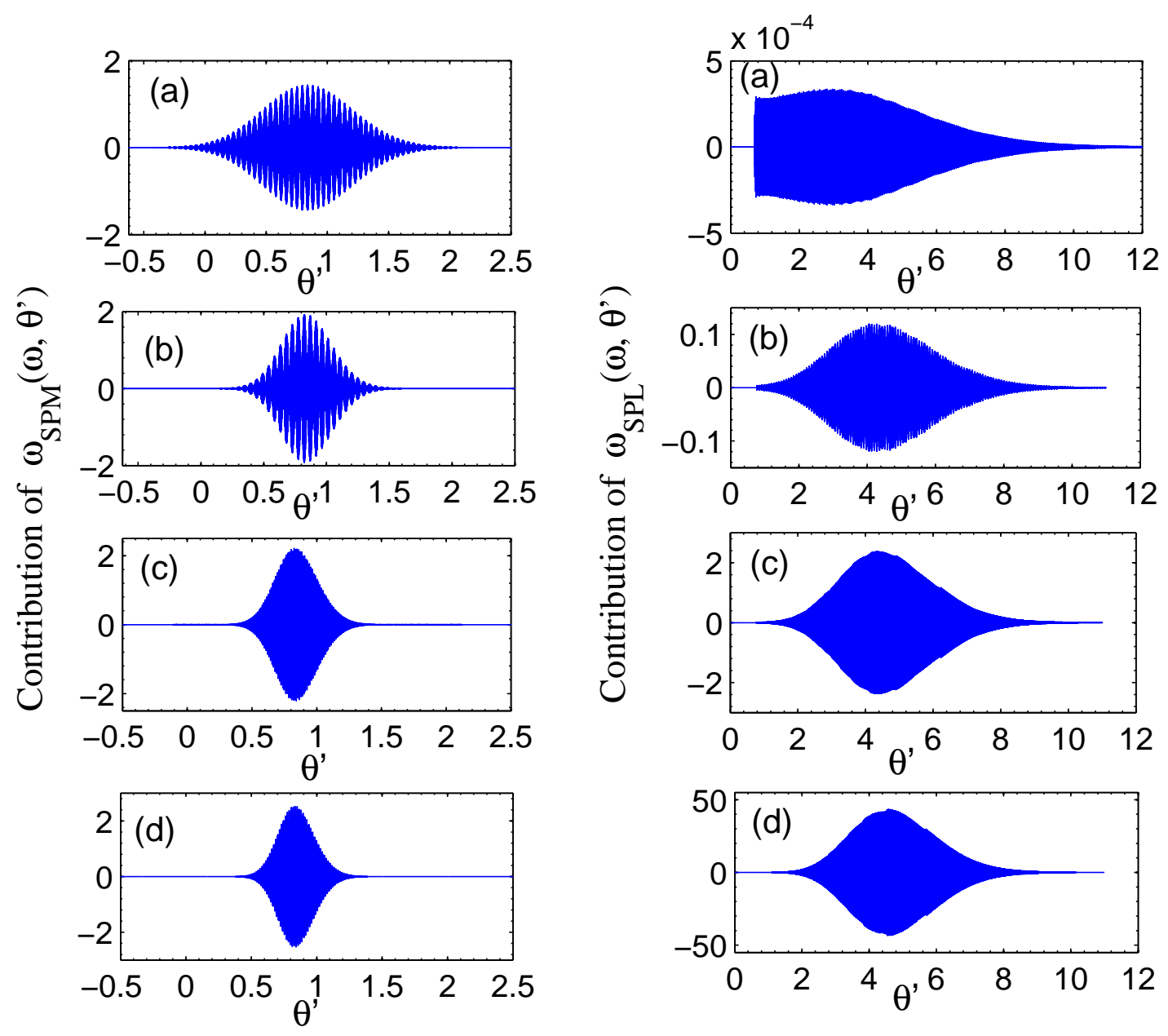

Figure 4.9: Saddle point contributions. The left column corresponds to the contribution of $\left(\omega_{S P, M}\right)$ and the right column corresponds to the contribution of $\left(\omega_{S P, L}\right)$. Cases (a-d) refer to propagation length of $25,44.5,54$ and $63(\mathrm{~cm})$ inside the ammonia vapor cells, respectively.

plified. Since the rate of amplification for the subluminal components is much more significant than the rate of amplification for the superluminal component, the output pulse is no longer dominated by the superluminal component after longer propagation distances.

The time domain behavior of the total field for the four cases is evaluated by adding the contributions of all the saddle points as described by Eq 4.14. This is depicted in Fig 4.10. The dashed lines refer to the case of a companion pulse traveling the same distance in vacuum for comparison.

In order to quantitatively characterize the arrival time for the pulses propagating in the AGD medium, as compared to their counterpart in vacuum, the center of mass 

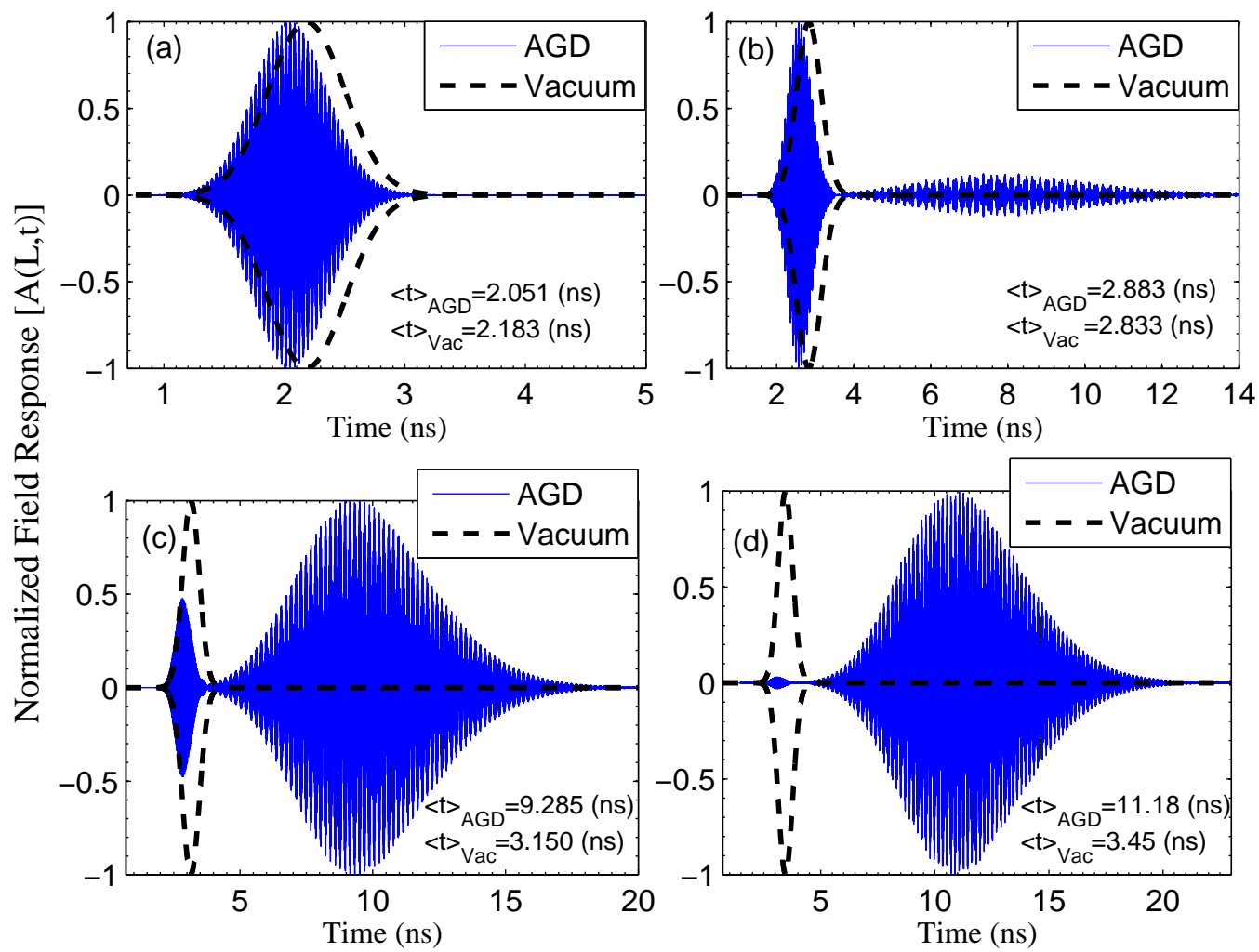

Figure 4.10: The output field $A(L, t)$. The black dotted curves correspond to the case of vacuum and $\langle t\rangle$ denotes the pulse arrival time. Cases (a-d) refer to propagation lengths of 25, 44.5, 54 and $63(\mathrm{~cm})$, respectively.

calculations are used. The expectation of the arrival time denoted as $\langle t\rangle$ is listed for cases (a-d) in Fig 4.10and is expressed as follows:

$$
\langle t\rangle=\frac{\hat{u} \cdot \int_{-\infty}^{\infty} t \mathbf{S}(\mathbf{z}, \mathbf{t}) d t}{\hat{u} \cdot \int_{-\infty}^{\infty} \mathbf{S}(\mathbf{z}, \mathbf{t}) d t} .
$$

The term $\mathbf{S}(z, t)$ denotes the Poynting vector of the propagating field and $u$ is a unit vector along the normal direction with respect to the detector surface. In this analysis, we assume normal incidence for the energy flow. Figure 4.10 shows that the pulse, after propagating $25(\mathrm{~cm})$ in the AGD medium, exhibits time advancement as compared to the companion pulse in vacuum. This is attributed to the fact that the superluminal components are dominant at this propagation distance as previously discussed. However, 
at longer penetration depths inside the ammonia vapor medium, cases (b) through (d), the subluminal components become more pronounced and the pulse arrival time in the AGD medium is retarded as compared to the companion pulse in vacuum. This implies that the center of mass for a superluminal pulse is retarded after propagating longer distances. Here, the cutoff distance is equal $\sim 43(\mathrm{~cm})$, in agreement to the prediction in Eq. 4.4. The transition of a superluminal pulse to a subluminal, in an inverted-atom medium, is usually accompanied with pulse broadening. For a Gaussian excitation, the pulse width is proportional to the variance and can be described as $\sigma^{2}=\left[\left\langle t^{2}\right\rangle-\langle t\rangle^{2}\right]$. Consequently, the pulse broadening factor, $\Gamma$, can be expressed as:

$$
\Gamma=\frac{\left[\left\langle t^{2}\right\rangle-\langle t\rangle^{2}\right]_{A G D}}{\left[\left\langle t^{2}\right\rangle-\langle t\rangle^{2}\right]_{\text {Vac }}} .
$$

From the definition in Eq. 4.16, intervals within which $\Gamma<1$ implies pulse compression while $\Gamma>1$ implies pulse broadening. For the cases (a-d) considered in this section, the pulse broadening factor $\Gamma$ is equal to $0.9323,32.15,99.93$ and 68.972 , respectively. Pulse broadening can be attributed to the fact that the spectral components that lie within the subluminal region experience large gain and differential delay.

In this section, it has been shown how the propagation distance imposes fundamental limitations on superluminal pulse propagation; where the dynamics associated with superluminal pulse transition to a subluminal regime (at longer propagation distances) has been discussed. In the next section, we consider Gaussian pulses with different spectral widths at a fixed distance to show that an analogous behavior takes place.

\subsection{Physical Dynamics of a Superluminal Pulse at different spectral widths}

In this section, we consider Gaussian pulse propagation at a fixed propagation depth ( $\mathrm{L}$ $=25(\mathrm{~cm})$ ) while varying the temporal width of the pulse. In order to study the effect of pulse width on superluminal propagation, we consider four different Gaussian pulses with temporal width $2 T$ that is equal to $0.9(n s), 0.7(n s), 0.5(n s)$, and $0.3(n s)$, respectively. The exact locations of the saddle points of $\phi\left(\omega, \theta^{\prime}\right)$ are numerically computed at each instant of time $\left(\theta^{\prime}\right)$ and is plotted over the isometric contours of $\operatorname{Re} \phi\left(\omega, \theta^{\prime}\right)$ for each of 
the four pulse widths as shown in Fig $4.11|4.12,4.13| 4.14$.

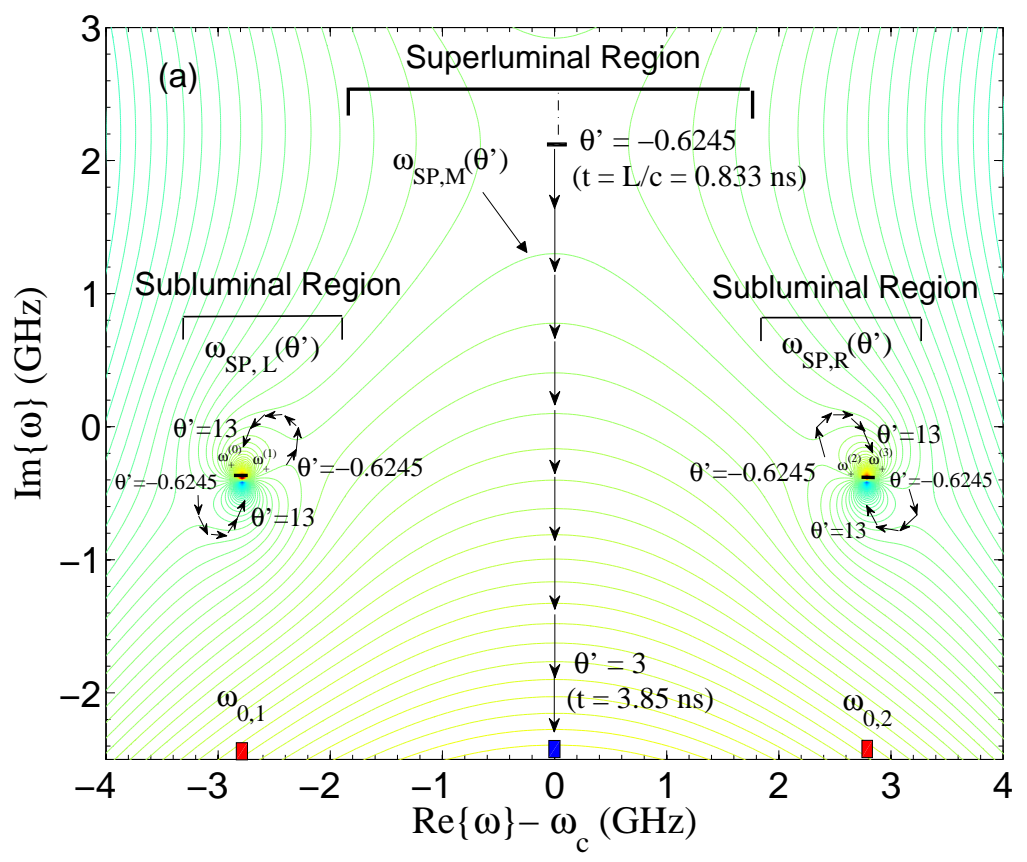

Figure 4.11: Path of saddle points $\omega_{S P}$ as $\theta^{\prime}$ increases at a fixed propagation distance $=25(\mathrm{~cm})$ inside the ammonia vapor for Gaussian pulse with an initial pulse width $2 \mathrm{~T}=0.9(n s)$.

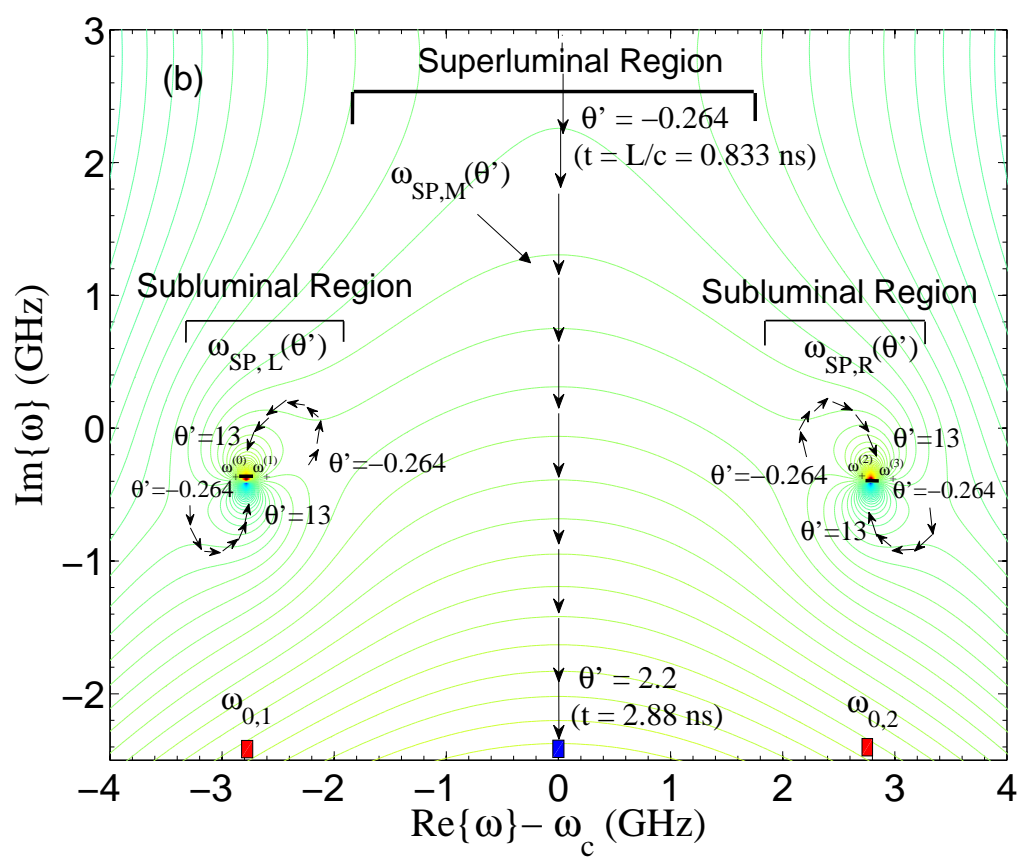

Figure 4.12: Path of saddle points $\omega_{S P}$ as $\theta^{\prime}$ increases at a fixed propagation distance $=25(\mathrm{~cm})$ inside the ammonia vapor for Gaussian pulse with an initial pulse width $2 \mathrm{~T}=0.7(n s)$. 


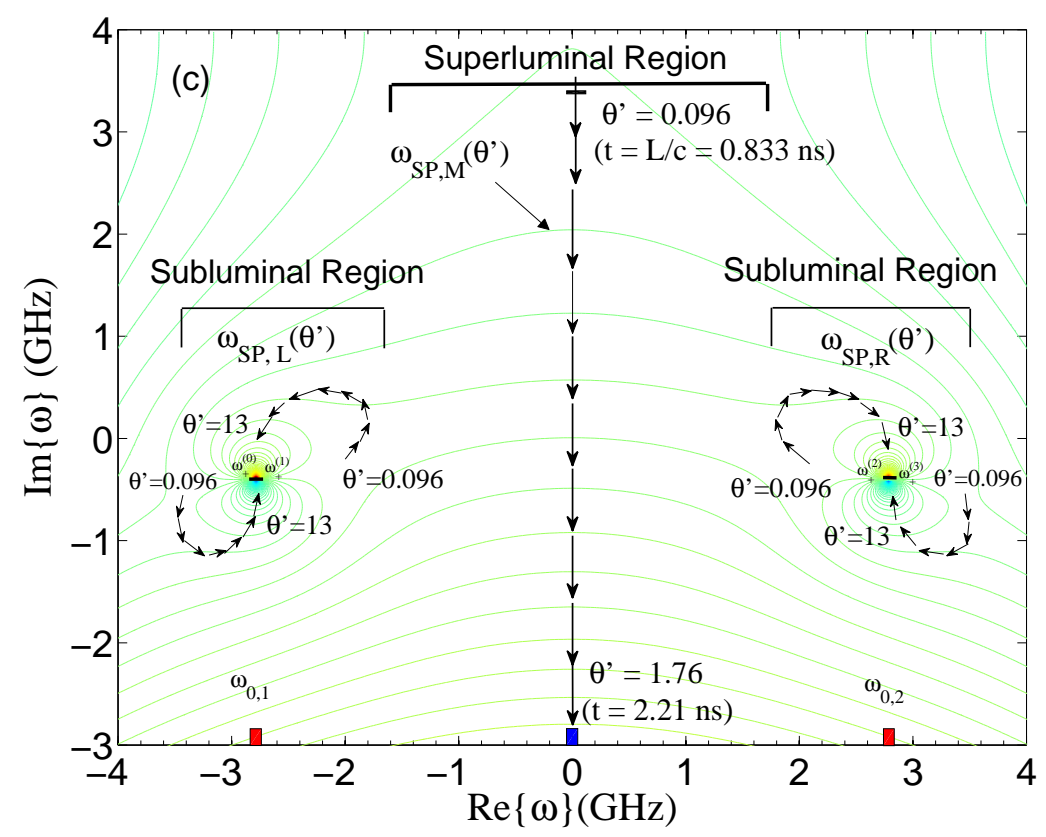

Figure 4.13: Path of saddle points $\omega_{S P}$ as $\theta^{\prime}$ increases at a fixed propagation distance $=25(\mathrm{~cm})$ inside the ammonia vapor for Gaussian pulse with an initial pulse width $2 \mathrm{~T}=0.5(n s)$.

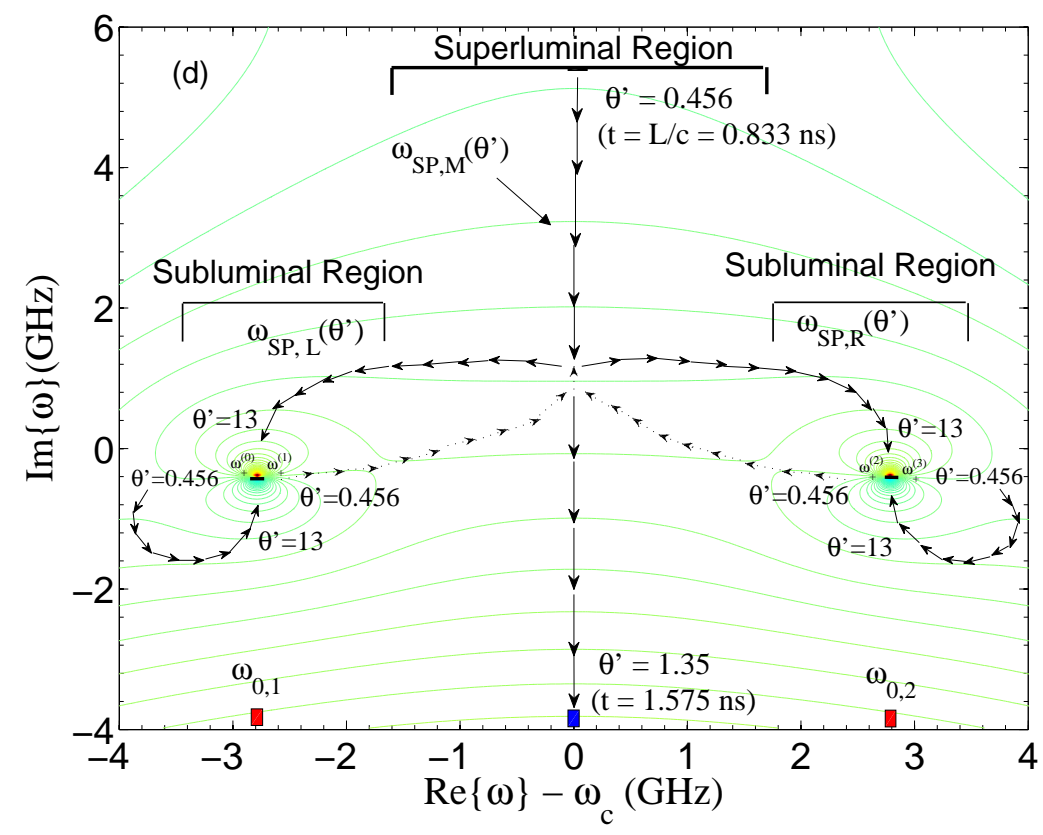

Figure 4.14: Path of saddle points $\omega_{S P}$ as $\theta^{\prime}$ increases at a fixed propagation distance $=25(\mathrm{~cm})$ inside the ammonia vapor for Gaussian pulse with an initial pulse width $2 \mathrm{~T}=0.3(n s)$.

The plots depicted in Fig 4.11 4.12 4.13 4.14 correspond to the four different pulse widths $[2 \mathrm{~T}=0.9(n s), 0.7(n s), 0.5(n s)$, and $0.3(n s)]$, respectively. Clearly, for a pulse 
with an initial spectrum well fitted within the superluminal region such as that depicted in Fig 4.11, the output field is dominated by the contribution of the middle saddle points $\omega_{S P, M}$ which follows a vertical path downwards at the carrier frequency $2.4166 \times 10^{15}$ $(\mathrm{rad} / \mathrm{sec})$ in the middle of the superluminal group delay regime. The other saddle points denoted as $\omega_{S P, L}$ and $\omega_{S P, R}$, both of which lie in the subluminal frequency region, have minimal (negligible) contribution to the construction of the total field.

Due to the transform limited nature of Gaussian pulses, as the pulse width is reduced the initial spectral width is subsequently increased and it starts leaking outside the gain medium bandwidth. Accordingly, the interaction of the pulse side bands with the gain doublet becomes more pronounced. This is evident in the cases depicted in 4.12 through 4.14 in which the circular path of the saddle points $\omega_{S P, L}$ and $\omega_{S P, R}$ becomes more spread out around the branch cuts as the temporal width is decreased. In extreme cases, such as the one depicted in 4.14 , the path of the subluminal saddle points $\omega_{S P, L}$ and $\omega_{S P, R}$ may even coalesce into a second order saddle point at the location of the carrier frequency before branching out towards the branch cuts. As such the contribution of the corresponding saddle points becomes more significant.

In all four cases, the path of the steepest descent saddle points in the subluminal region, denoted as $\omega_{S P, L}$ and $\omega_{S P, R}$, imply that not only is the contribution of the saddle points significantly delayed with respect to the dominant part of the pulse associated with $\omega_{S P, M}$ but it suffers from chirping as well. For the path of $\omega_{S P, L}$, the instantaneous frequency is decreasing in the direction of the branch cut $\left(\omega_{+}{ }^{(0)}, \omega_{+}{ }^{(1)}\right)$; whereas for the path of $\omega_{S P, R}$, the instantaneous frequency is increasing in the direction of the other branch cut $\left(\omega_{+}^{(2)}, \omega_{+}^{(3)}\right)$. A similar argument is valid for the cases considered in section 4.4 as well.

The decomposed contributions of $\omega_{S P, M}$, denoted as $A_{\omega_{S P, M}}\left(\theta^{\prime}\right)$ and $\omega_{S P, L}$, denoted as $A_{\omega_{S P, L}}\left(\theta^{\prime}\right)$ are plotted in Fig 4.15 for the four cases. Clearly, as the temporal pulse width is decreased, the contributions of $\omega_{S P, L}$ (and $\omega_{S P, R}$ ) become more significant and the output pulse is no longer dominated by the superluminal component (evaluated from the contribution of $\left.\omega_{S P, M}\right)$. In addition, evidently, the rate of amplification for the subluminal components (contributions of $\omega_{S P, L}$ and $\omega_{S P, R}$ ) is much more significant than the rate of amplification of the superluminal component (contribution of $\omega_{S P, M}$ ) as in section 

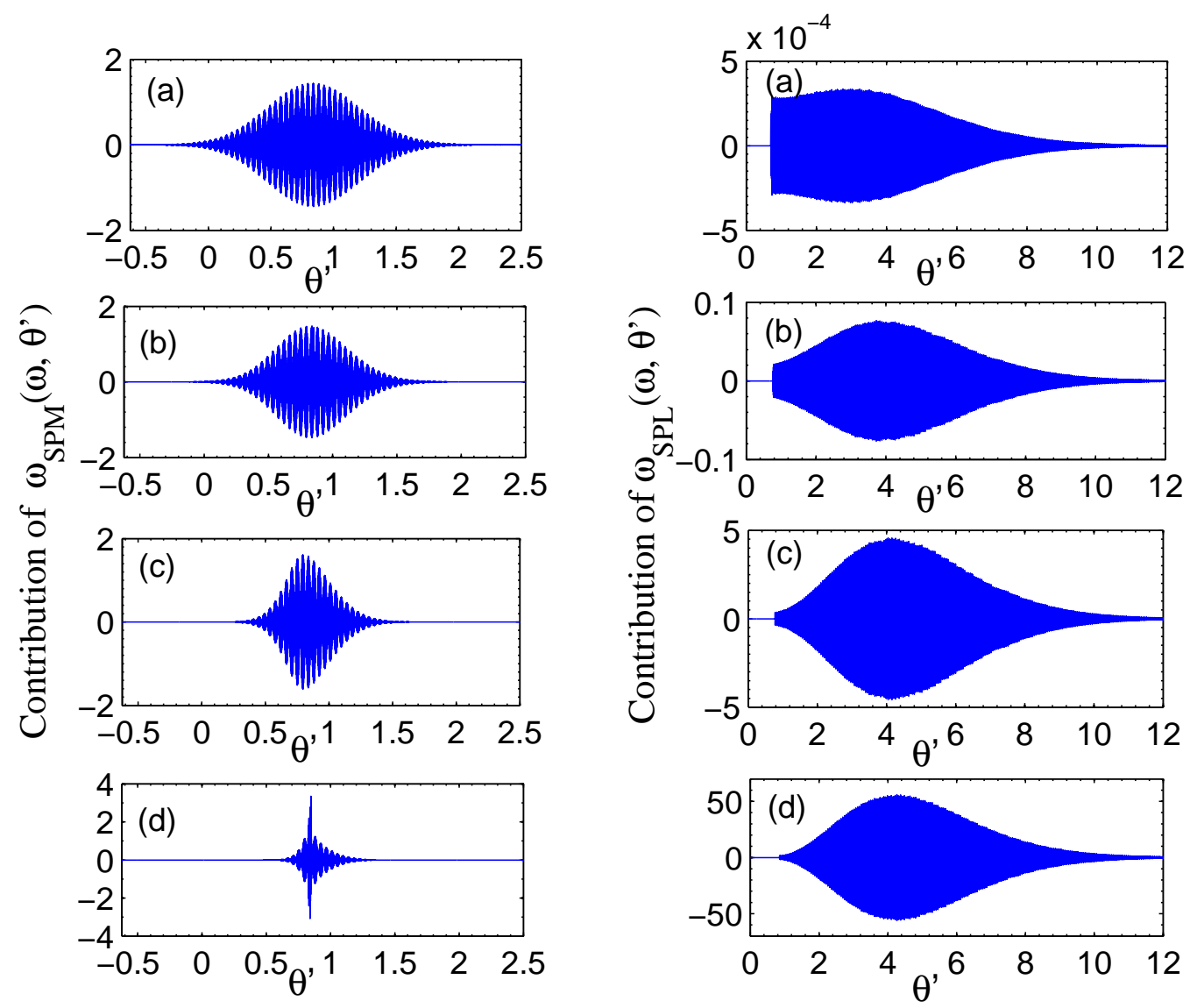

Figure 4.15: The decomposed contribution of the saddle point of the phase function $\phi\left(\omega, \theta^{\prime}\right)$. The left column corresponds to the contribution of the middle saddle points $\left(\omega_{S P, M}\right)$ and the right column corresponds to the contribution of the Left saddle points $\left(\omega_{S P, L}\right)$. Cases (a-d) refer to initial pulse width $2 \mathrm{~T}=0.9(n s), 0.7(n s), 0.5(n s)$, and $0.3(n s)$, respectively.

4.4. This behavior is generic and represent a duality with the case of a passive medium in which the rate of absorption in the superluminal region is much more pronounced as compared to the absorption endured within the subluminal frequency range as illustrated in Fig 3.4 .

The total field response can be directly calculated by adding the contributions of all the saddle points. The total fields are illustrated in Fig 4.16, for cases (a-d), and compared to the case of vacuum as a reference. The expectation of the arrival time of energy are also listed in the same figure for cases (a-d). Clearly, the arrival times for the pulses in the AGD medium in cases (a) and (b) are advanced as compared to the respective arrival time in vacuum. This is a consequence of the fact that the superluminal components are dominant in those cases. In cases (c) and (d), however, the subluminal components 

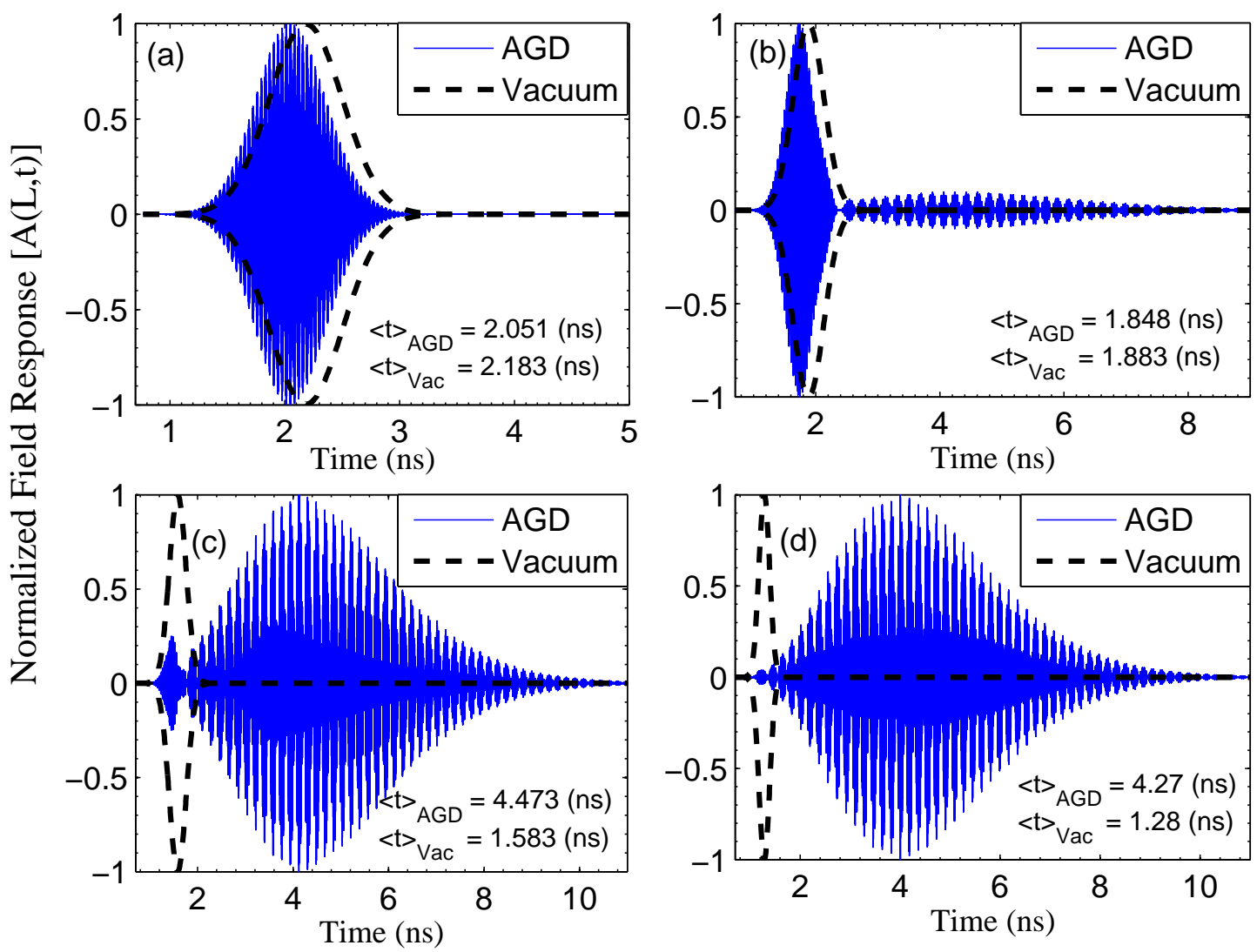

Figure 4.16: The total output field response at a propagation distance $25(\mathrm{~cm})$ for the case of an AGD medium (ammonia vapor). The field is obtained by adding the contributions of $\omega_{S P, L}, \omega_{S P, M}$ and $\omega_{S P, R}$. The black dotted curves correspond to the case of vacuum and $\langle t\rangle$ denotes the pulse arrival time. Cases (a),(b),(c) and (d) refer to initial pulse width $2 \mathrm{~T}=0.9(n s), 0.7(n s), 0.5(n s)$ and $0.3(n s)$, respectively.

are more pronounced and the pulse arrival times in the AGD medium are retarded as compared to a companion pulse propagating in vacuum, confirming the prediction of Eq. 4.4. The pulse broadening factor $\Gamma$ for the four cases (a-d) is $0.9323,11.3037,89.17$ and 228.25, respectively. It is noticed that pulse broadening always precede the transition to subluminal regime. For instance, in case (b), the broadening factor is 11.3037 while the arrival time is still superluminal. This behavior is general and applies to the cases considered in section 4.4 as well. 


\subsection{Conclusion}

In this Chapter, we have presented a simple closed form analytic expression to predict the cutoff distance at which a superluminal pulse becomes subluminal. The expression provides the fundamental limitations of superluminal pulse propagation in light of factors such as the propagation distance, pulse spectrum, and medium dispersion strength. Furthermore, the method of steepest descent has been utilized to investigate the timefrequency dynamics associated with the transition from the superluminal to the subluminal regime, in an inverted medium. This has been done by decomposing a superluminal pulse into its superluminal and subluminal components under different configurations. Cases in which a superluminal pulse evolves to the subluminal regime at longer propagation depths (and narrower pulse widths) have been presented to verify the approximate analytic expression. This evolution is attributed to the subluminal spectral components that dominate over the pulse during propagation. 


\section{Chapter 5}

\section{Velocity of Detectable Information in Faster-than-light Pulses}

In this chapter, the velocity of detectable information (signal velocity) in a medium capable of supporting abnormal (superluminal or negative) group velocities is calculated. This is carried out by tracking the time instant at which the signal-to-noise ratio (SNR) at the detector output reaches a predetermined threshold while considering the total classical and quantum noise of the channel in addition to the detector noise. Furthermore, the method of steepest descent is incorporated to include pulse reshaping effects which have been widely overlooked. By studying the behavior of SNR as a function of both space and time, the present analysis predicts the existence of a cut off distance beyond which signal velocity of a superluminal pulse is delayed as compared to its counterpart in a vacuum channel of the same length. Finally, the interplay between the relative strength of the medium generated noise and the detector noise and its effect on signal velocity is discussed.

\section{$5.1 \quad$ Introduction}

Although from a fundamental point-of-view relating the information velocity of a pulse with its strictly luminal front is valid; however, from a practical point-of-view this approach is not completely satisfactory for the following reasons. 
First, in many practical situations the field strength associated with the front and precursor oscillations is extremely small and well below the detection level. This makes the detection of the points of non-analyticity (singularities) if not impossible, certainly very challenging [31].

Second, information cannot be viewed as a localized quantity (e.g. points of nonanalyticity, alone) as its detection requires the act of a detector and a window of time associated with arrival of enough power (sufficient amount of photons) to trigger a detection process.

Third, information, its propagation, and its detection are stochastic processes, in which each subsystem of a communication system plays an important role. Each subsystem introduces a certain amount of noise that imposes considerable effects on the stochastic nature of the information and its velocity. In short, any definition for detectable information should account for the associated noise from the channel and the non-ideal detector 1 .

According to the three aforementioned considerations, a more operational approach for calculating the signal velocity — which depends on the signal-to-noise ratio $(S N R)$ - has been widely utilized in the literature [39, 40, 56]. For such cases, the $S N R$ is expressed (as a function of time) in the following general form

$$
S N R(t)=\frac{P_{\text {sig }}(t)}{P_{\text {MedNoise }}(t)+P_{\text {DetNoise }}(t)},
$$

where $P_{\text {sig }}(t)$ is the signal power, $P_{\text {Det Noise }}(t)$ is the noise associated with the detector circuit and $P_{\text {MedNoise }}(t)$ is the total classical and quantum noise associated with the medium at a given propagation distance.

In such approach, the signal velocity (velocity of detectable information of a pulse) is related to the time instant at which the signal power exceeds the noise floor $(S N R=0$ $\mathrm{dB})$. Using this operational definition, the possibility of reducing signal detection latency using AGD media has been demonstrated both theoretically [40, 56] and experimentally [29].

\footnotetext{
${ }^{1}$ In comparative analysis, in which a pulse propagating through free space is compared to a pulse traveling through an AGD channel, pulse generation effects can be neglected owing to the assumption that both pulses are generated under the same circumstances.
} 


\section{$5.2 \quad$ Literature shortcomings}

Although the approach of relating signal velocity to the propagation speed of a point with a constant $S N R$ at the pulse front has been previously used in the literature; however, there are still some un-addressed shortcomings:

First, there has been very limited investigation for the effect of the propagation distance on the $S N R$ and the corresponding signal velocity. In a temporally dispersive medium that supports superluminal (or negative) group delay, the amount of group delay and pulse reshaping - in terms of compression (or broadening) and amplification (or absorption) - is a function of the medium length. Such effects have a direct impact on the temporal distribution of the propagating energy in the medium which directly affects the description of $P_{s i g}(t)$ in the numerator of Eq. 5.1. Likewise, the total quantum and classical noise sources associated with the medium depends closely on the medium gain $g(\omega)$ which in turn is a function of the length. For an inverted medium, as will be considered in this analysis, there are four main sources of noise generated in the medium: spontaneous emission noise, beating noise between the signal and the amplified spontaneous emission, shot noise in addition to self beating of the amplified spontaneous emission [39, 40]. Each of these noise terms, which are combined in the term $P_{\text {MedNoise }}(t)$ in Eq. 5.1, evolve differently on the channel based on the propagation distance. Accordingly, any approach that investigates whether superluminal propagation can reduce signal detection latency without considering the optimum medium length (that maximizes the $S N R$ ) is not entirely conclusive. This has been the case in [39, 40]. This has left the behavior of signal velocity during propagation and its dependency on the medium length unexplored.

Second, superluminal group velocity is in essence a pulse reshaping phenomenon and hence pulse reshaping in terms of compression (or broadening) plays an important role and must be taken into account. The work in [39, 40], while discussing SNR, do not take the pulse reshaping into account and in fact their theoretical analysis assume that the output pulse is a mere shifted version of the input pulse by the amount of the group delay. Moreover, this mere time shift can have nonphysical consequences on the behavior of the front which always must obey a luminal speed. Unfortunately, from the text in [39, 40] 
it is not clear if the authors in the aforementioned references have properly compensated for the luminal speed of the front or not. We find such approach not satisfactory at a physical level.

In this chapter, the aforementioned shortcomings are addressed; where the formulation of the $S N R$ is extended as a function of both time and space in order to study the effect of propagation distance on the signal velocity, while including the exact pulse reshaping effects.

\subsection{Approach}

We consider an inverted medium that exhibits AGD and account for the output signal power in addition to the medium generated noise and the detector noise. The $S N R$ is expressed as a function of both time and space and is solved for the time instant at which SNR reaches $0 \mathrm{~dB}$. For a simple on-off keying communication scheme in which information is carried by either a bit-1 or bit-0, the $0-\mathrm{dB} S N R$ level defines the arrival of the information window at different space points during field propagation. In effect, this can be visualized as the following: a detector travels along with the pulse, measuring and reporting the corresponding SNR at each instant of time for each point in space, while measurements leave the pulse intact for the future measurements and the detector keep no memory of the previous measurements. The detector is thus scanning the signal along its propagation in the medium to find the optimal medium length that maximizes the SNR. One can use this approach for both a channel (medium) with AGD and a vacuum channel to investigate the relative advancement (or retardation) of the signal as a function of the medium length. In order to account for the pulse reshaping effects, the method of steepest descent (saddle point analysis) is incorporated.

It is shown that - despite the superluminal delay and medium gain - there exists a cut off distance beyond which the signal velocity is delayed with respect to its counterpart in vacuum. Finally, it is shown that the relative strength of the detector noise with respect to the medium noise can affect the signal velocity.

This chapter is organized as follows: in section 5.4, we introduce the dispersive AGD medium and perform the respective calculations for the output field response for such 
medium by using the method of steepest descent. In section 5.5, we consider the classical and quantum noise sources associated with the AGD medium and the non ideal detector. Additionally, we formulate the $S N R$ for both the AGD medium and vacuum channel as a function of pace and time in order to discuss the impact of the medium length on the signal velocity. In section 5.6, we discuss the effect of the detector noise and medium length and its relation to the detectable information in light of previous analyses in the literature. Finally, the conclusion is presented in section 5.7.

\subsection{Field Calculation in Causal Dispersive Medium}

For a medium with AGD, we consider a double resonance medium with gain such as ammonia vapor cells at the GaAlAs laser wavelength $(\lambda=780(\mathrm{~nm}))$. The index of refraction for such a medium is described as[21]:

$$
n(\omega)=\sqrt{1+\frac{\omega_{p, 1}^{2}}{\omega^{2}-\omega_{0,1}^{2}+2 i \delta \omega}+\frac{\omega_{p, 2}^{2}}{\omega^{2}-\omega_{0,2}^{2}+2 i \delta \omega}} .
$$

Where $\omega_{p, j}$ and $\omega_{0, j}(j=1$ or 2$)$ are the plasma and resonance frequencies associated with the first and second resonances, and $\delta$ is the phenomenological line-width $(\sim 400$ $(M H z))$. For each of the resonances, the typical condition $\delta<\omega_{p, j}<\omega_{0, j}$ is satisfied. Such medium has a causal response and, consequently, the Kramers-Kronig relations are valid. The frequency dependent index of refraction and the group delay of the gain medium, as a function of detuning, are depicted in Fig. 5.1.

The ammonia medium exhibits abnormal group delay (with $\sim 0.085$ ( $n s$ ) advancement) with a flat response within a detuning of $4(G H z)$ around $\omega_{c}=2.4166 \times 10^{15}$ $(\mathrm{rad} / \mathrm{sec})(\lambda=780(\mathrm{~nm}))$.

We chose an input modulated Gaussian pulse centered at the carrier frequency $\omega_{c}=$ $2.4166 \times 10^{15}(\mathrm{rad} / \mathrm{sec})$ and an initial pulse width $2 T=0.8(\mathrm{~ns})$ centered at $t_{0}>0$ $(\sim 3 T)$. The pulse is excited at the plane $z=0$ and propagates inside a medium with an index of refraction that is given in Eq. 5.2. The input Gaussian pulse is written in the form:

$$
f(t)=e^{-\left(\frac{t-t_{0}}{2 T}\right)^{2}} \sin \left(\omega_{c} t\right) .
$$




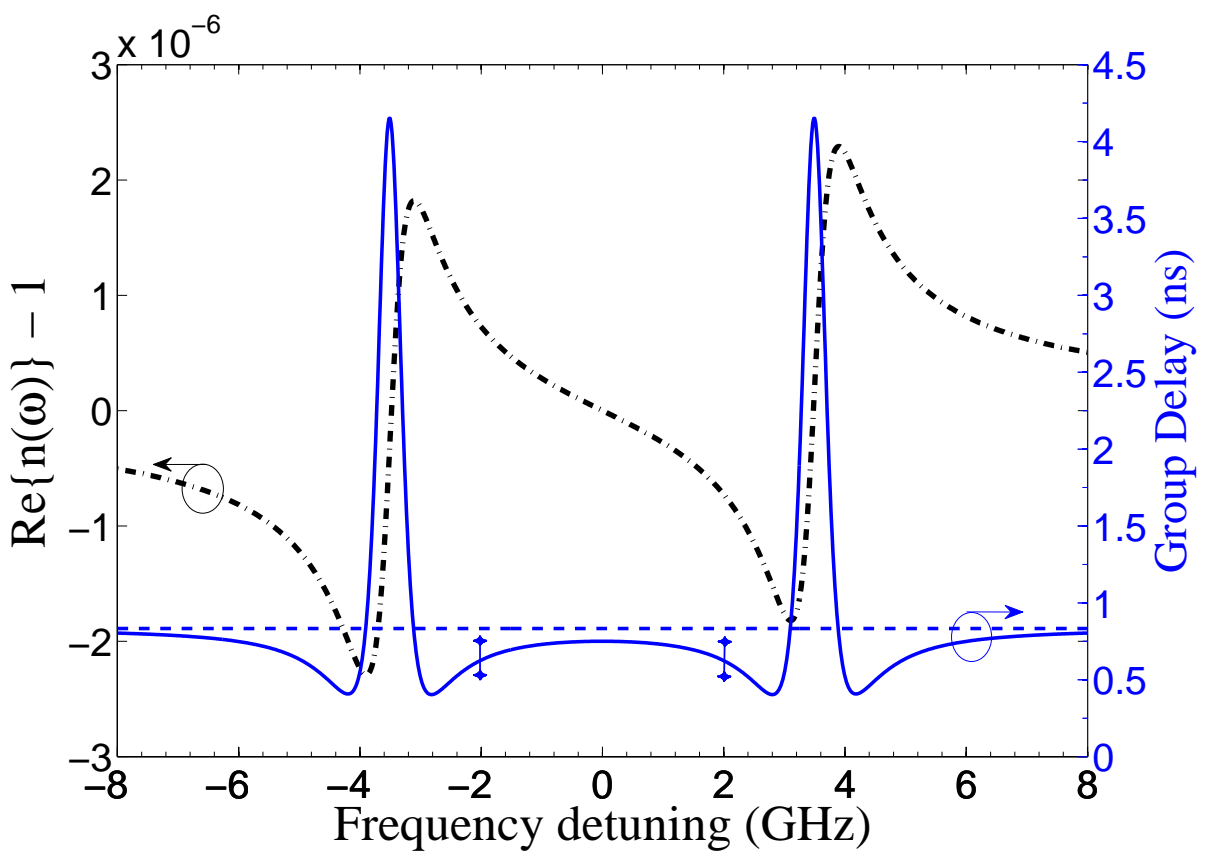

Figure 5.1: Index of refraction $n(\omega)$ and group delay as a function of detuning (the blue dashed line denotes the luminal group delay in vacuum). The vertical arrows mark the spectral width of the input pulse.

For such signal, the initial spectrum is expressed as:

$$
F(\omega)=\sqrt{\pi} T e^{\frac{T^{2}\left(\omega-\omega_{c}\right)^{2}}{4}} e^{-i \omega_{c} t_{0}}
$$

Accordingly, the general description of the field $E(z, t)$ is obtained by solving the integral:

$$
E(z, t)=\frac{1}{2 \pi} \operatorname{Re}\left[i \int_{C} \tilde{F} e^{z \phi\left(\omega, \theta^{\prime}\right) / c} d \omega\right]
$$

Where $\tilde{F}=\sqrt{\pi} T e^{-i \omega_{c} t_{0}}$ is the spectral amplitude and $\phi\left(\omega, \theta^{\prime}\right)=i \omega\left[n(\omega)-\theta^{\prime}\right]-$ $\frac{c T^{2}}{4 z}\left(\omega-\omega_{c}\right)^{2}$ is the unified phase function for a Gaussian pulse. The term $\theta^{\prime}=c\left(t-t_{0}\right) / z$ represents the dimensionless space-time parameter [4]. At a given propagation distance $z$, different values of $\theta^{\prime}$ correspond to different instants of time.

As discussed in Chapter 3, the total field is calculated by deforming the integration contour in Eq. 5.5 over the path of steepest descent of the phase function $\left[\phi\left(\omega, \theta^{\prime}\right)\right]$. Such deformation is achieved by adding the contributions of the saddle points $\left(\omega_{S P}\right)$ that satisfy $\frac{d \phi\left(\omega=\omega_{S P}, \theta^{\prime}\right)}{d \omega}=0$. This approach provides useful insights to the time-frequency dynamics of the field and accounts for the precursor oscillations and pulse reshaping. 
Figure 2 depicts the paths of the different saddle points in the complex frequency domain as a function of $\theta^{\prime}$. Each saddle point contribution $\left(A_{\omega_{S P}}\right)$ to the total field can be expressed in the following closed form expression (which was provided in Eq. 3.9) and written here for convenience [47]:

$$
A_{\omega_{S P}}\left(\theta^{\prime}\right)=\sqrt{\frac{c}{2 \pi z}} \operatorname{Re}\left[\frac{i \tilde{F} e^{\frac{z}{c} \phi\left(\omega_{S P}, \theta^{\prime}\right)}}{\sqrt{\frac{-d^{2}}{d \omega^{2}} \phi\left(\omega_{S P}, \theta^{\prime}\right)}}\right] .
$$

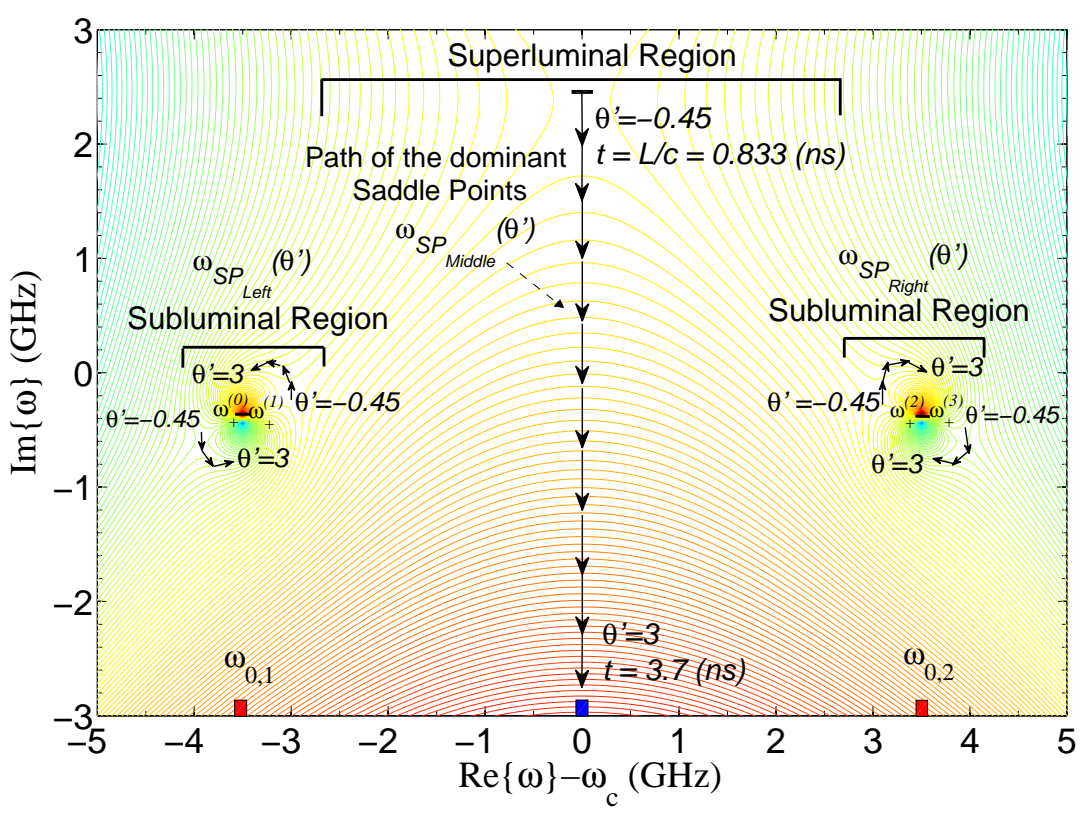

Figure 5.2: Path of the saddle points of $\left[\phi\left(\omega, \theta^{\prime}\right)\right]$ as a function of $\theta^{\prime}$ at a distance $L=25(\mathrm{~cm})$ inside the ammonia vapor. The colored contours illustrates the $\operatorname{Re}\left[\phi\left(\omega, \theta^{\prime}\right)\right]$ evaluated at $\theta^{\prime}=-0.45$ (which corresponds to time $t=L / c)$. The first and second resonances are denoted by $\left(\omega_{0,1}\right)$ and $\left(\omega_{0,2}\right)$, respectively. $\left(\omega_{+}{ }^{(0)}, \omega_{+}{ }^{(1)}\right)$ and $\left(\omega_{+}{ }^{(2)}, \omega_{+}{ }^{(3)}\right)$ signify the branch cuts for the resonances $\omega_{0,1}$ and $\omega_{0,2}$.

Additionally, as has been shown in previous chapters, the method of saddle points has an additional advantage: it highlights the saddle points that contribute significantly to the construction of the pulse as opposed to saddle points with minimal contributions. In Fig. 5.2, the dominant saddle point, denoted as $\omega_{S P_{\text {Middle }}}$, follows the vertical path moving downwards at the carrier frequency $\omega_{c}=2.4166 \times 10^{15} \mathrm{rad} / \mathrm{sec}$ in the middle of the superluminal region. The other saddle points, denoted as $\omega_{S P_{\text {Left }}}$ and $\omega_{S P_{\text {Right }}}$, follow a circular path towards the branch cuts $\left(\omega_{+}{ }^{(0)}, \omega_{+}{ }^{(1)}\right)$ and $\left(\omega_{+}{ }^{(2)}, \omega_{+}{ }^{(3)}\right)$, respectively. These latter saddle points are located within the subluminal region and their contributions to the total generated pulse energy is negligible. As seen later, the information content of the 
pulse is recoverable without the need to detect these spectral components at the receiver side. For an input pulse with a broader spectral width (or at longer distances), the contributions of the saddle points in the subluminal region $\left(\omega_{S P_{\text {Left }}}\right)$ and $\left(\omega_{S P_{\text {Right }}}\right)$ become more pronounced and may even lead to a scenario in which the pulse becomes subluminal 2. In this analysis, we consider propagation lengths at which the pulse remains in the superluminal regime (at propagation distances shorter than z in Eq.4.5).

All saddle points contributions are added and the medium response $A(L, t)$ is calculated as depicted in Fig. 5.3. The result is verified using the inverse Laplace method [54] and shows a perfect match as demonstrated in the same figure 3 .

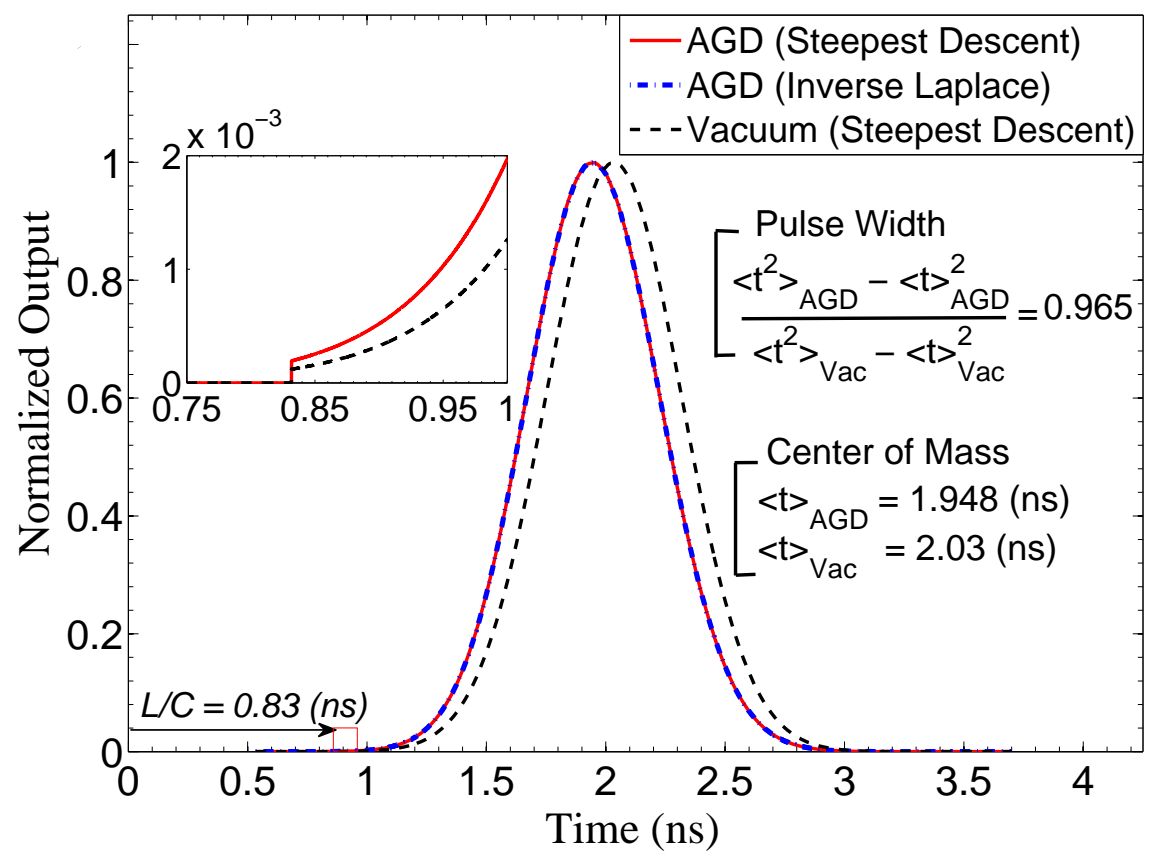

Figure 5.3: Normalized medium response at propagation distance $L=25(\mathrm{~cm})$. The insets depict the arrival of the pulse front after duration $L / c$ in compliance with Einstein's causality.

The normalized Gaussian pulse, after traveling $25(\mathrm{~cm})$ inside the ammonia vapor, experiences slight compression (3.5\%) and exhibits peak advancement $(10.25 \%$ of the FWHM). The calculations of the expectation of arrival time, following Ref. [46], demonstrate 0.0842 ( $n s$ ) advancement for the AGD medium in agreement with our results.

\footnotetext{
${ }^{2}$ The detailed saddle-point dynamics for different pulse widths and propagation depths (including superluminal and subluminal cases) has been presented in the previous chapter.

${ }^{3} \mathrm{~A}$ brief overview on the algorithm used to calculate the field using this numerical method can be found in further details in Appendix $\mathrm{C}$
} 
The inset in Fig. 5.3 depicts the arrival of the front in both ammonia and vacuum (of the same length $L$ ) with a luminal delay $(t=L / c)$ in compliance with Einstein's causality and Sommerfeld.

Thus far the output response of the channel (dispersive medium) has been evaluated. At this point, the detection process needs to be included in our formulation. To do so, we must account for the noise generated in both the channel and the detector and calculate its impact on the $S N R$ (discussed next).

\subsection{Signal Velocity in an Inverted Medium with Superluminal Delay}

In order to calculate the signal velocity in an inverted medium, the noise generated in the medium and the detection process are taken into account. Since the ammonia vapor is acting as a gain medium, we include both the quantum and classical sources of noise in our analysis. We consider the following sources: shot noise $\left(P_{\text {Shot }}(t)\right)$, spontaneous emission noise $\left(P_{A S E}(t)\right)$, mutual beat noise between the signal and amplified spontaneous emission $\left(P_{S i g-A S E}(t)\right)$, and self-beat noise of the amplified spontaneous emission $\left(P_{A S E-A S E}(t)\right)$. The expressions for these noise sources are derived from [39, 40] and extended here to be a function of both space and time. As such, the shot noise is given as:

$$
P_{\text {shot }(z, t)}=2 e P_{\text {sig }}(z, t) \Re B_{e} .
$$

The term $P_{\text {sig }}(z, t)$ is the instantaneous power incident on the detector surface and can be

expressed as $P_{\text {sig }}(z, t)=\frac{1}{t} \int_{0}^{t} E(z, \tau)^{2} d \tau$; where $E(z, \tau)$ is the optical field after propagating for an arbitrary distance $z$. $\Re$ characterizes the detector responsivity which translates the incident optical power into electric current $(\Re=0.1(A / W))$, $e$ is the electron charge, and $B_{e}$ is the detection bandwidth $\left(B_{e}=5 \mathrm{KHz}\right)$. The spontaneous emission noise can be written as:

$$
P_{A S E}(z, t)=N_{s p} \hbar \nu \frac{1}{t} \int_{0}^{t} \int_{w}\left[g(z, \omega)^{2}-1\right] d \omega d \tau,
$$

where $N_{s p}$ is the spontaneous emission factor, $\hbar \nu$ is the energy per photon, $g(z, \omega)$ is the equivalent medium gain as a function of frequency and length. 
The self beat noise of amplified spontaneous emission is described as:

$$
P_{A S E-A S E}(z, t)=\frac{1}{t} \int_{0}^{t} F(z, \tau)^{2} d \tau
$$

Where $F(z, t)$ is the noise correlation of the amplified spontaneous emission expressed as $F(z, t)=N_{s p} \hbar \nu \int_{w}\left[g(z, \omega)^{2}-1\right] e^{-i \omega t} d \omega$. Lastly, the beat noise between the signal and the amplified spontaneous emission is expressed as:

$$
P_{\text {Sig-ASE }}(z, t)=\frac{2}{t} \int_{0}^{t}\left[F(z, \tau) P_{s i g}(z, \tau)\right] d \tau .
$$

Additionally, we account for the detector noise in terms of its thermal noise and dark current; where dark current arises from the mobility of electron charges in the detector circuit even in the absence of detected signal. For a photomultiplier detector, the $\operatorname{SNR}(z, t)$ at the detector output can be expressed as a function in time and space as follows [39, 40, 56]

$$
S N R(z, t)=\frac{2 G^{2}\left[P_{s i g}(z, t) \Re\right]^{2}}{G^{2}\left[2 e\left(i_{d}+P_{s i g}(z, t) \Re+P_{A S E}(z, t) \Re\right)+\frac{4 P_{A S E-A S E}(z, t) \Re^{2}}{B_{o}}+\frac{2 P_{S i q-A S E}(z, t) \Re^{2}}{B_{o}}\right] B_{e}+\frac{4 K T B_{e}}{R}} .
$$

Here, $G$ is the detector photo-multiplication gain $(G=80), i_{d}$ is the dark current $\left(i_{d}=10^{-14}(A), K\right.$ is Boltzmann constant, and $T$ is the temperature in Kelvins ( $T=$ $300(k)) . B_{o}$ denote the optical bandwidth $\left(B_{0}=10(G H z)\right.$; and $\frac{4 K T B_{e}}{R}$ represents the thermal noise associated with the equivalent detector resistance $R(R=500 \Omega)$. For the vacuum channel, the noise sources only include the shot noise, thermal noise, and dark current.

At each space point $z$, the $S N R(z, t)$ in Eq. 5.11 is solved for the time instant that corresponds to $0 \mathrm{~dB}$ (arrival of detectable information) for both the case of ammonia vapor and a vacuum channel. The time difference between the arrival of detectable information in the ammonia vapor medium and its arrival in vacuum is denoted as $\Delta \tau_{\text {Sig }}$ and is depicted in Fig. 5.4 .

A negative value for $\Delta \tau_{S i g}$ implies faster signal detection in the ammonia vapor medium. Figure 5.4 implies that there exists a range of space points within which the signal velocity is faster in the AGD medium than its counterpart in vacuum (as depicted 


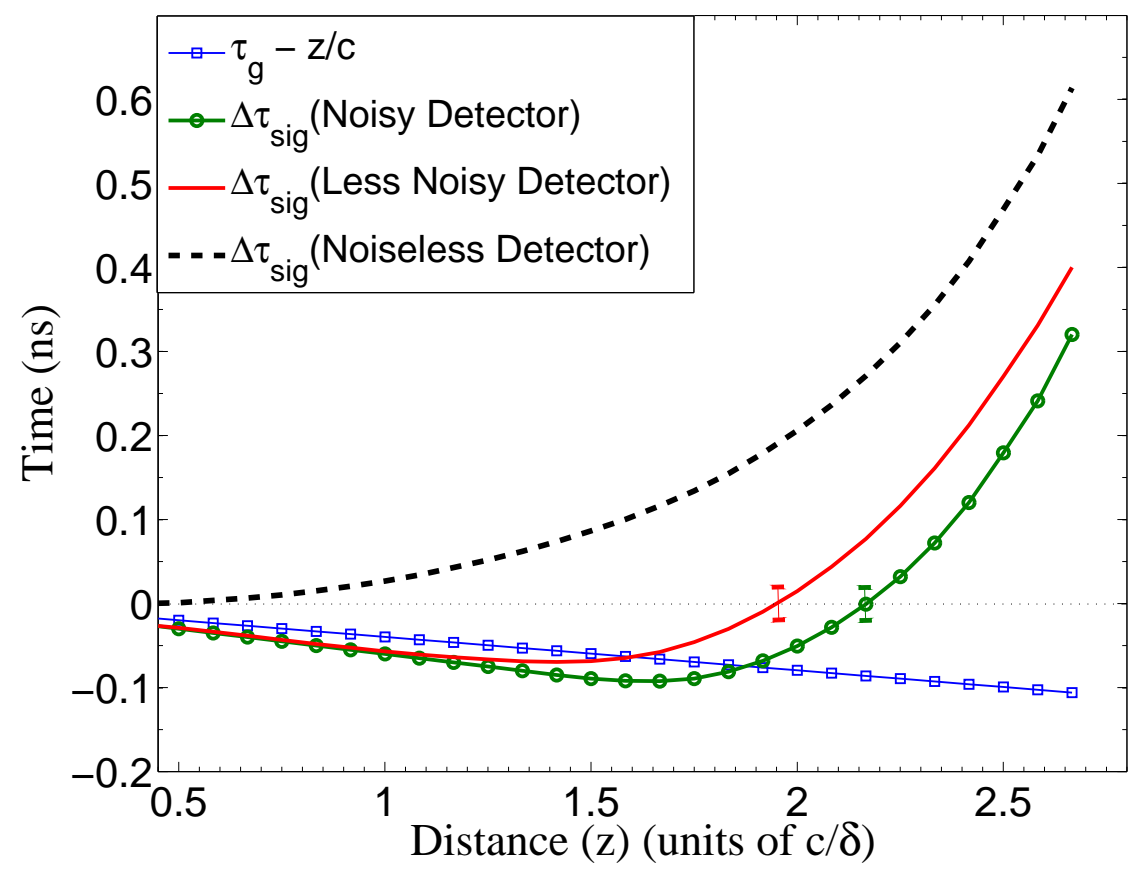

Figure 5.4: Relative signal delay in ammonia vapor cells with respect to a vacuum channel as a function of propagation distance in units of $(c / \delta)$. The relative signal delay is compared with the corresponding relative group delay (denoted by the blue line). This comparison is carried out for three different noise levels at the detector side.

by the negative values of the black and red lines).

Additionally, it is shown that there exist a cutoff distance beyond which the signal velocity is delayed as compared to vacuum despite the superluminal group delay (as marked by the vertical black line). Accordingly, the medium length plays an important role in the proper interpretation of signal velocity and should not be overlooked in any experimental setup. This will be further discussed in the next section.

Moreover, the noise level of the detector (in terms of its thermal noise) determines whether signal velocity can be time advanced with respect to a vacuum channel. This is also depicted in Fig. 5.4 in which the noisy and less noisy detectors correspond to detector equivalent resistance $\mathrm{R}=0.5(k \Omega)$, and $5(k \Omega)$, respectively. It is shown that signal latency can be reduced when the detector noise is much more pronounced. For instance, when the detector noise level is reduced, the cut off distance (at which signal velocity is delayed) shifts to a shorter length. Whereas in the limit of noiseless detector, it is shown that signal velocity is always delayed with respect to vacuum in agreement 
with Ref. [39] (in which the detector noise was omitted). This is illustrated by the green line which acquires a non-negative value for all space points.

In this section, the effects of both the medium length and detector noise on signal velocity have been highlighted. In the next section, we show the temporal behavior of SNR at three different space points to further demonstrate the effect of the detector noise and the medium length. Furthermore, we discuss the notion of detectable information in light of previous points-of-view in the literature.

\subsection{Discussion}

In this section, the $S N R$ - as a function of time - is investigated at three different propagation distances (which correspond to three different space points in Fig. 5.4). This emphasizes the effect of the detector noise and the medium length on signal velocity. Moreover, the results are tied together with previous interpretations in the literature.

First we consider propagation distance below the cutoff $(L=2.083(c / \delta)$ which corresponds to $25(\mathrm{~cm})$ inside the ammonia vapor). The $S N R(t)$ associated with the input Gaussian pulse of Eq. 5.3, after propagation for distance $L$ is calculated at the detector output and is depicted in Fig. 5.5 (red curve). The $S N R(t)$ of the same input pulse at the output of the same detector, but this time traveling $2.083(\mathrm{c} / \delta)(25(\mathrm{~cm}))$ in vacuum is also calculated and shown in Fig. 5.5 (solid black curve). Figure 5.5 shows that the front and high frequency oscillations associated with it (Sommerfeld precursors) maintain a strictly luminal speed in both media in agreement with Sommerfeld's and Brillouin's prediction [1, 2, 3]. This is demonstrated by the fact that the earliest response of both media occurs simultaneously after $t=L / c(0.83(n s))$ in compliance with Einstein causality.

From a communication system point-of-view, although the front or points of nonanalyticity, in which genuine information of a wavepacket can be encoded, arrive luminaly; however, the association of information with these points is troublesome. For instance, in Fig. 5.5, the SNR at onset of the pulse arrival (the front) is as low as $-175 \mathrm{~dB}$ which makes its detection extremely difficult if not impossible 4 . Therefore, associating information

\footnotetext{
${ }^{4}$ In fact, depending on how smoothly the input pulse has been turned on, the SNR associated with the front and precursors can be even much lower than $-175 \mathrm{~dB}$.
} 


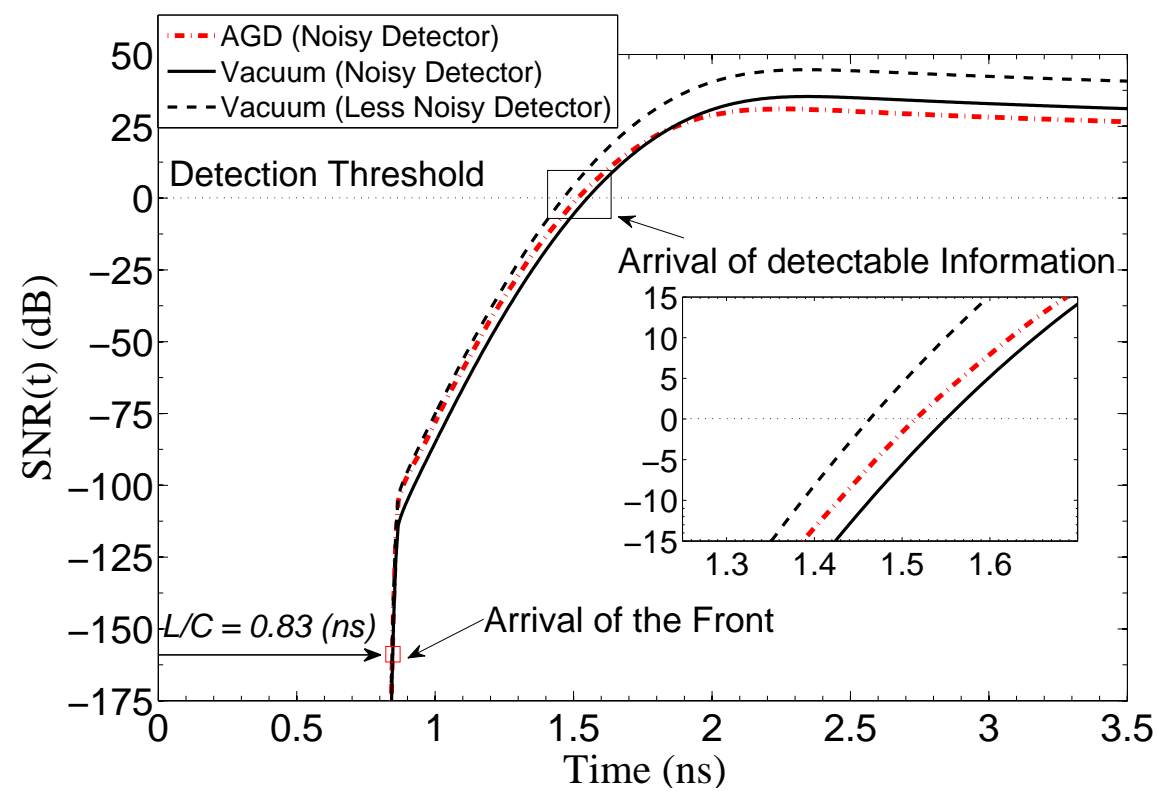

Figure 5.5: Signal-to-noise ratio as a function of time at a propagation depth of $25(\mathrm{~cm})$ in the ammonia vapor. The inset shows the information window at vicinity of the detection threshold as compared to a vacuum channel of the same length.

and its velocity with the front (or singularities) when such field quantities cannot be measured makes little practical sense. For most communication systems the decision threshold is set at $0 \mathrm{~dB}$, when signal power is equal to the noise power. In light of Fig. 5.5, it seems, apparently, that the information velocity associated with the AGD channel is superluminal at the $0 \mathrm{~dB}$ level - i.e., $S N R(t)$ of the AGD channel is advanced in time as compared to the $S N R(t)$ of vacuum. However, these results must be interpreted properly as discussed below.

The vacuum channel in the above discussion should no longer be regarded as "pure vacuum" but rather a vacuum loaded with a noisy detector. As such, the $S N R$ of the AGD medium is compared to this perturbed vacuum channel. If the noise power associated with the detector is reduced - for example, by increasing the detector equivalent resistance (R, in Eq. 5.11) from $0.5(k \Omega)$ to $5(k \Omega)$ - the $S N R(t)$ of the loaded vacuum channel will be shifted in Fig. 5.5 from the solid black line to the dashed black line on the same figure, while the $S N R(t)$ for AGD channel remains almost unchanged (dominated by its inherent noise). In other words, any advantage that the AGD channel would have provided in terms of advanced detection of information washes away in comparison to the vacuum channel with less noisy detector. This effect is in agreement with the prediction 
in section 5.5 (Fig. 5.4). Consequently, compliance to Einsteins's causality is imposed without the need to refer to the unmeasurable front. It should be noted that the aforementioned finite increase in the detector equivalent resistance is always a possibility; although it may not be pursued in all cases due to the cost considerations. In such cases, this analysis clearly shows that there is an advantage to be gained in reducing the signal detection latency by utilizing AGD channels in particular applications in agreement with other studies which has considered electronic circuits [14, 56].

The presented results bring together three points-of-view:

First, it indicates that the recent experimental observations by Vogl et al. [29] in early detection of information encoded in an optical pulse traveling in an AGD medium as compared to free space, is a consequence of operating in a situation similar to the one described by the pair of solid black and solid red curves in Fig. 5.5.

On the other hand, the observation by Chiao et al. [39], on the limitation imposed by quantum fluctuation on the advancement of the information in AGD medium, is a consequence of operating in a situation similar to the one described by the pair of solid red and dashed black curves in Fig. 5.5 .

Third, previous assertions by Sommerfeld and Brillouin [1, 2, 3], in associating information velocity with the velocity of front and singularities, corresponds to the theoretical case of having an infinitely sensitive detector which in Fig. 5.5 pushes the detection threshold to the bottom of the figure at $-175 \mathrm{~dB}$.

Finally, the deciding factor on which scenario applies to which experimental observation depends on the relative strength of the detector noise as compared to the total noise of the AGD channel in addition to the length of the medium.

The distance that an optical pulse, encoded with information, travels through an AGD medium greatly affects the velocity by which information is transmitted. This has been shown in section 5.6 (Fig. 5.4). To further investigate this dependency, Fig. 5.6 shows the temporal behavior of $S N R(L, t)$ for the AGD and vacuum channels at three different lengths where the detector used is the same as the noisy detector depicted by the red line in Fig. $5.5(R=0.5(k \Omega))$.

As the propagation depth increases, any advantage of the AGD channel (in early detection of information) over the vacuum channel of the same length washes away. This 


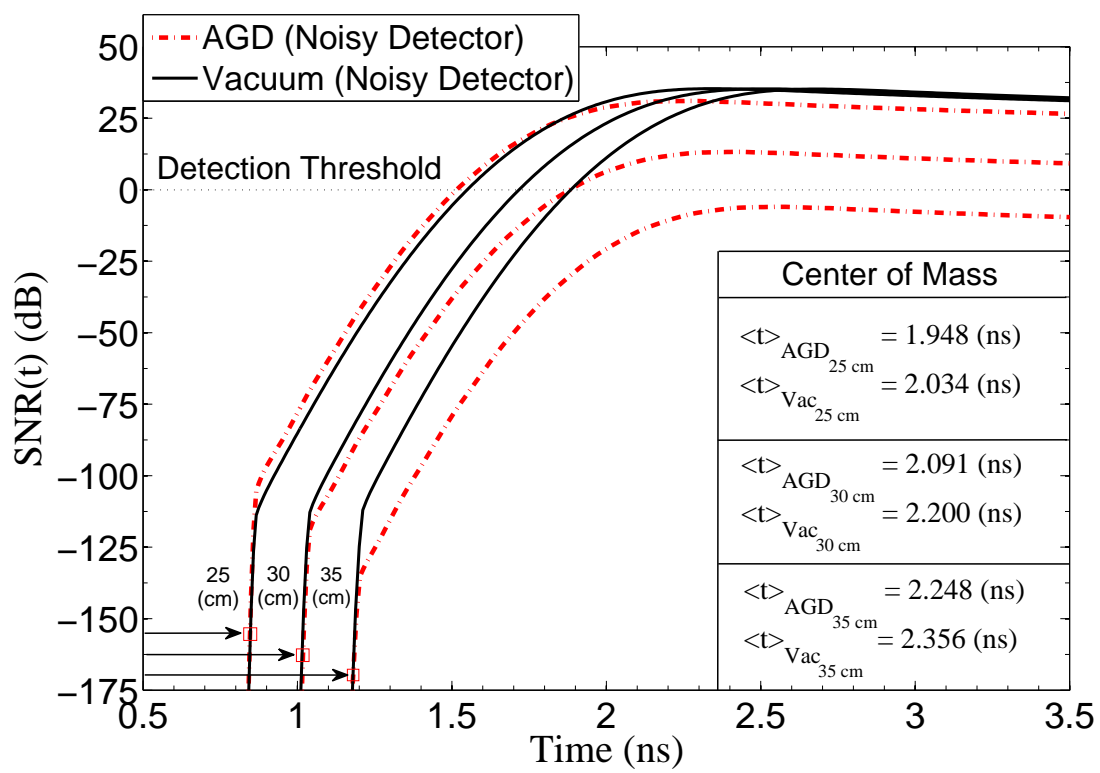

Figure 5.6: $S N R(L, t)$ at different propagation depths $L=2.083(c / \delta), 2.5(c / \delta)$ and $2.917(c / \delta)$ (which corresponds to 25, 30 and $35(\mathrm{~cm})$, respectively) in an AGD medium and compared to a vacuum channel. Evidently, information velocity is retarded in an AGD medium despite the exhibited superluminal behavior indicated by the center of mass calculations.

diminished advantage can be attributed to the increased quantum noise generated in the longer gain medium as compared to the perturbed vacuum.

It should be added that unlike Ref. [41], for all cases depicted in Fig. 5.6, the group velocity remains superluminal as indicated by the center of mass calculations in the inset of the figure.

\subsection{Conclusion}

In this chapter, from a practical communications system point-of-view, we have studied the notions of group, front, and information velocities in a medium exhibiting AGD with gain. Using the saddle point analysis we have considered propagation of an optical pulse, encoded with information, in ammonia vapor cells at different depths while including pulse reshaping effects. It is observed that, depending on the relative strength of the noise power in the AGD channel as compared to the detector noise, the AGD medium can be used to reduce the information detection latency as compared to a vacuum channel of equal length. Additionally, it has been shown that, for a superluminal pulse, there exist a cut off distance beyond which the signal velocity is delayed with respect to vacuum. 
In all such cases, it has been demonstrated that Einstein's causality is preserved without the need to refer to the practically undetectable front. It is worth noting that a similar approach have been applied by the author of this thesis in the Microwave domain as well reaching similar conclusions 5 .

\footnotetext{
${ }^{5}$ For further detailed, see reference [42]
} 


\section{Chapter 6}

\section{Detectable Information and}

\section{Discontinuities in Causal pulses}

Points of non-analyticity (discontinuities) in a pulse - which are strictly luminal under all circumstances - have always been associated with its genuine information content. From a fundamental point-of-view, these discontinuities carry the unpredictable behavior of the pulse and cannot be extrapolated using a Taylor expansion, for instance. In this chapter, we consider pulses encoded with points of non-analyticity and we further investigate the behavior of these points in fast and slow light applications. We then apply the approach presented in Chapter 5 in order to calculate the detectable information content of these pulses. It is found that, unlike the case of fast light, it is possible to obtain subluminal pulses with multiple discontinuities and the respective information content remains below the detection threshold (not practically detectable) even after the arrival of all the nonanalytic points at the detector. We thus argue that, from a practical point-of-view, information should not be entirely associated with the pulse discontinuities. As such, the distinction between the discontinuities of a pulse, its energy transport and detectable information is demonstrated. Such analysis elaborates the fundamental capabilities and limitations of slow light applications. 


\subsection{Introduction}

It has been well established that the abnormal behavior encountered in a medium with superluminal (or negative) group delay is in full compliance with Einstein's causality. Such behaviors have been attributed to pulse reshaping and energy exchange between the pulse and the medium, as discussed in Chapter 1. It has also been shown that, unlike the pulse front, the input and output pulse peaks are non causally connected. This has been experimentally demonstrated in Ref. [32] in which a bandpass amplifier was used to achieve negative group delay. Although the input pulse was truncated at the position of its peak as depicted in Fig. 6.1; nevertheless, the 'time-advanced' output pulse maintained its shape with a well detectable peak after which it started to have an oscillatory (non meaningful) response. In a medium with a non-negligible length, such ringing should take place after a strictly luminal duration $\sim L / c[33$.

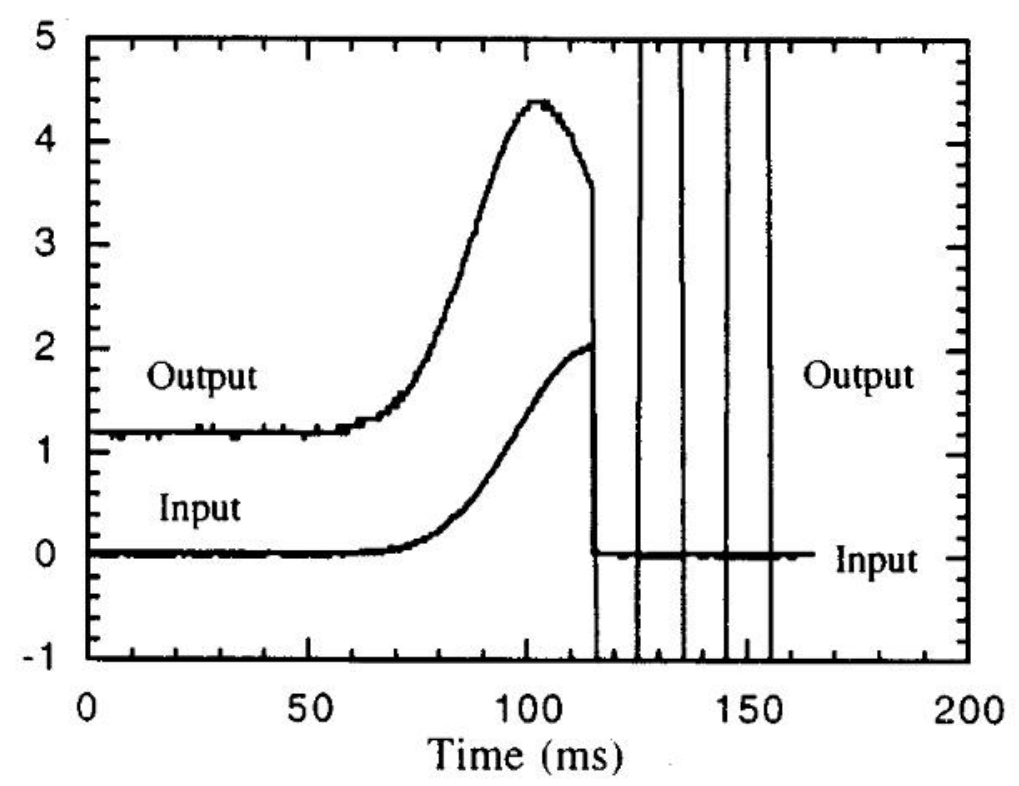

Figure 6.1: Input and output pulses of a negative group delay band pass amplifier. The discontinuity "kicks" the amplifiers, producing large-amplitude ringing. This ringing exceeds the scale of the graph and appears as vertical lines following the discontinuity. Input and output were acquired simultaneously with no averaging. Curves have been offset vertically for clarity [32].

This implies that, for a superluminal pulse, it is not possible to detect any useful information after the arrival of the pulse singularity at the output. Accordingly, information has been entirely associated with the pulse discontinuities. In this chapter, we investigate the dual effect in slow light (subluminal) propagation. It is shown that, unlike 
the case of fast light, it is possible to detect useful information in subluminal pulses even after the occurrence of the singularity at output of the medium. We thus show that pulse discontinuities cannot be used in general to quantify the detectable information of a pulse.

\subsection{Approach}

We consider causal Gaussian pulse propagation in a medium that exhibits slow (and fast) $v_{g}$. For such a medium, a singularity is introduced by terminating the pulse at the position of its peak, where a 'window' is generated. The boundaries of such window extend between two discontinuities; the first discontinuity is due to the transitional turn-on of the pulse whereas the second discontinuity is a result of the sudden pulse termination. In effect, this can be interpreted as if there are two windows that are superimposed on top of each other at the input of the medium. These two windows are: the aforementioned window (bounded by the two discontinuities) and the actual energy window due to the field excitation (pulse envelope). Initially, the boundaries of both windows are perfectly aligned before propagating inside the medium. Afterwards, during propagation, both windows travel at different speeds. For the case of slow light propagation, it is shown that the singularity due to pulse termination arrives at the output sooner than a considerable part of the subluminal energy of the input pulse. Nevertheless, the pulse maintains its shape even past the termination point and the remainder of the pulse is recoverable after the arrival of the singularity. On the other hand, when the same approach is applied to the case of pulse propagation in a vacuum channel or a medium with superluminal (or negative group delay), the truncation point at the output is received later after the arrival of the energy window and it is followed by non-meaningful ringing in agreement with Einstein's causality. Afterwards, we recall the metric introduced in Chapter 5, $S N R(t)$, in order to compare between the arrival of the discontinuities of the pulse as opposed to the arrival of the detectable information content.

This chapter is organized as follows: in section 6.3, we introduce the slow light medium considered in the analysis. In section 6.4, we use the finite difference time domain method (FDTD) to calculate the field response at the output of the slow light medium when the 
input pulse is truncated. In section 6.5, we incorporate the $S N R(t)$ analysis to compare between the arrival of discontinuities as opposed to the arrival of detctable information of pulses in slow light media. In section 6.6, we discuss the behavior of discontinuities for the case of superluminal propagation. Finally, the concluding remarks are provided in section 6.7 .

\subsection{Field Propagation in Causal Slow-light Medium}

Slow light applications have received considerable attention recently with regard to optical switching, data storage and signal processing [57, 58, 59, 60, 61, 62. For a medium that exhibits slow group velocity, we consider a causal passive Lorentzian medium with a single resonance. Such medium can be practically realized using AlGaN and the index of refraction of such a medium is expressed as:

$$
n(\omega)=\sqrt{1-\frac{\omega_{p}^{2}}{\omega^{2}-\omega_{0}^{2}+2 i \delta \omega}} .
$$

Here, $\omega_{p}$ and $\omega_{0}$ refer to the plasma and resonance frequencies, respectively, and $\delta$ is the phenomenological line-width. The numerical values of the medium parameters are listed Table 6.1.

Table 6.1: Numerical values for the parameters of the single resonance Lorenztian Model (AlGaN).

\begin{tabular}{|c|c|}
\hline$\omega_{0}$ & $1.921 \times 10^{14}(\mathrm{~Hz})$ \\
\hline$\omega_{p}$ & $3.328 \times 10^{14}(\mathrm{~Hz})$ \\
\hline$\delta$ & $9.756 \times 10^{12}(\mathrm{~Hz})$ \\
\hline
\end{tabular}

The real and imaginary parts of the index of refraction are plotted in Fig. 6.2 as a function of frequency.

For such medium with length $L$, the group delay $\left(\tau_{g}=L / v_{g}\right)$ is expressed as $\tau_{g}=\frac{-d \phi}{d \omega}$ where $\phi$ is the phase associated with the term $e^{-\omega \operatorname{Im}[n(\omega)] L / c}$. It is observed that the medium exhibits negative group delay in the frequency region around the resonance $\omega_{0}$ as depicted in Fig. 6.3. On the other hand, the frequency range around $\omega=0.477 \times 10^{14}(\mathrm{~Hz})$ corresponds to subluminal $\tau_{g}$. Evidently, at very low (and high) frequencies the medium exhibits strictly luminal behavior. 


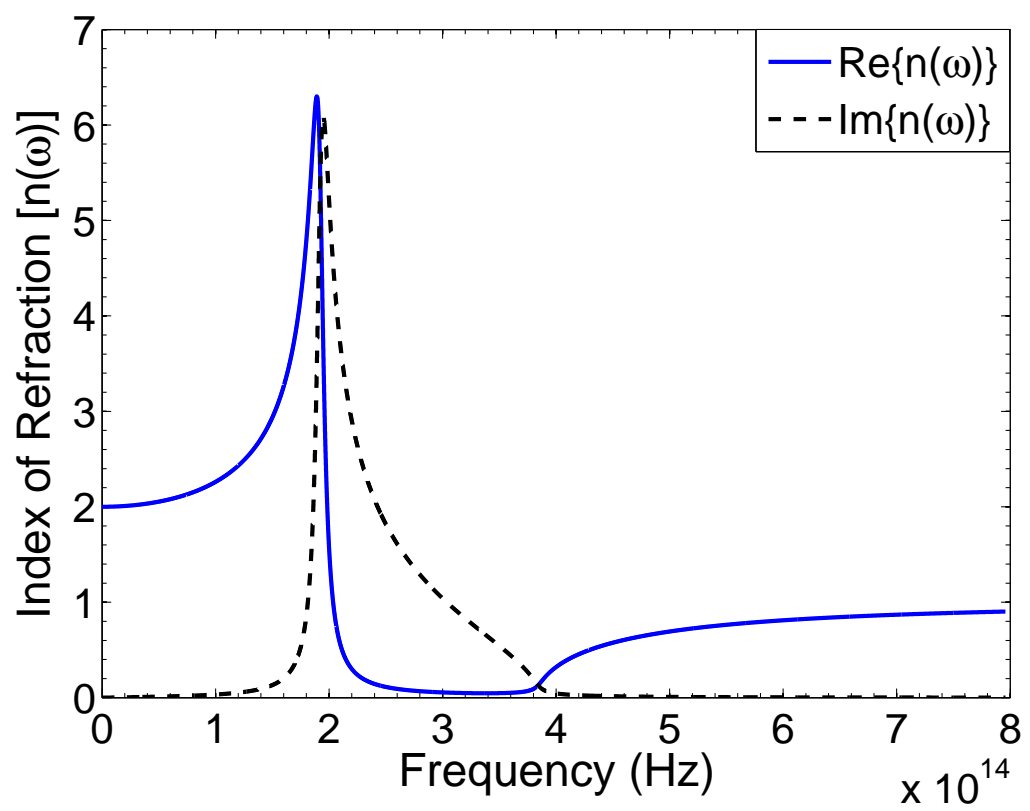

Figure 6.2: Real and imaginary parts of the index of refraction of AlGaN.

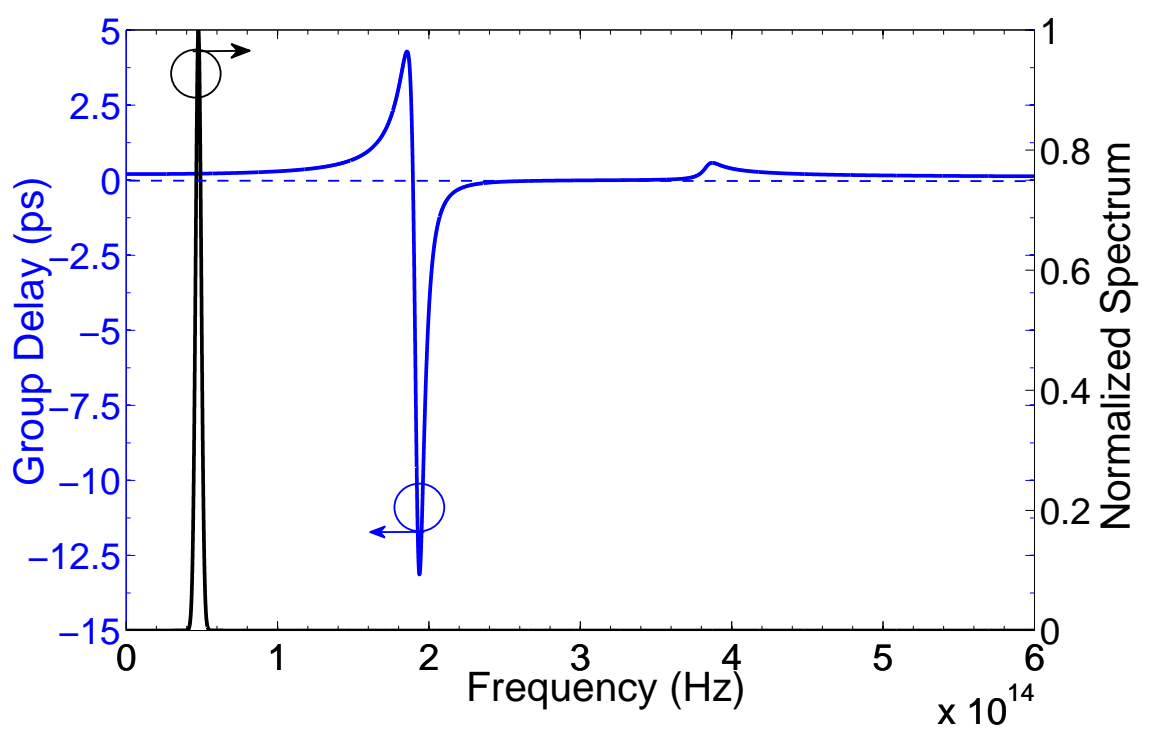

Figure 6.3: The input Gaussian spectrum is superimposed on the group delay $\left(\tau_{g}\right)$ plot. $\tau_{g}$ is calculated for a length $L=30(\mu \mathrm{m})$. The blue dashed line corresponds to $\tau_{g}$ in vacuum.

In this analysis, we chose an input Gaussian pulse with a carrier frequency centered at $47.7(\mathrm{THz})$ (in the near infrared region) and a pulse width $2 T$ (corresponds to the $e^{-1}$ amplitude) equal to $0.24(\mathrm{ps})$. The pulse peak interacts with the medium at time $t_{0} \sim 3 T$. The causal input pulse is expressed as: 


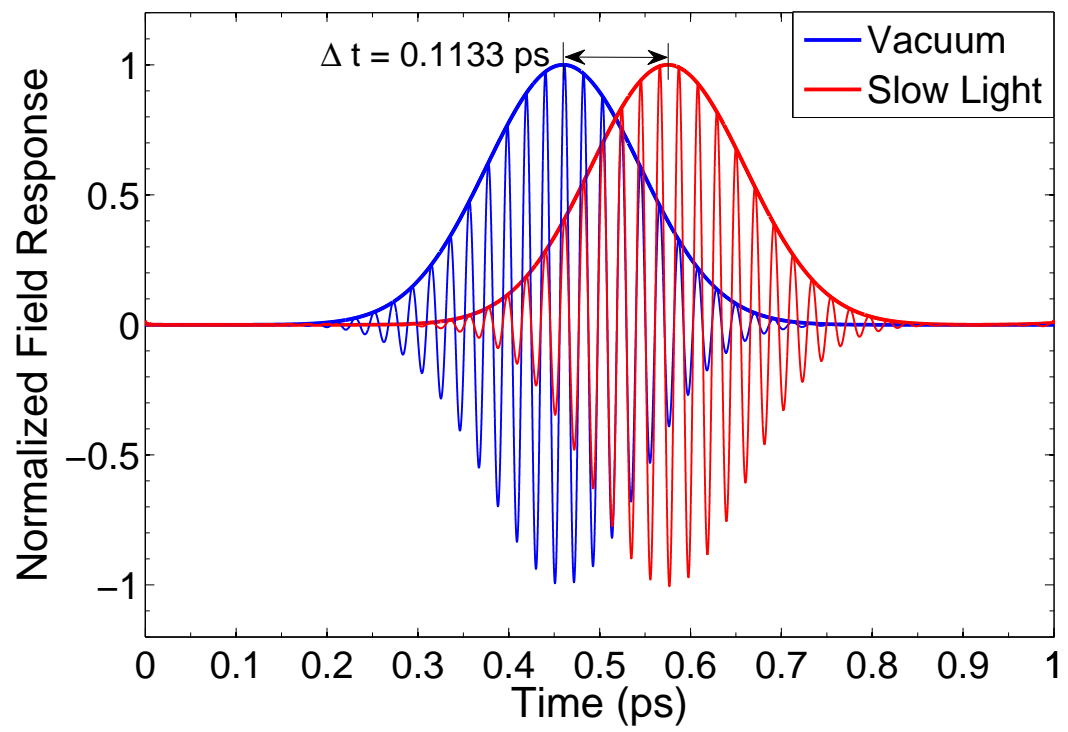

Figure 6.4: Pulse propagation in a medium with subluminal group delay $\tau_{g}$ and vacuum at a distance $L=30(\mu \mathrm{m})$. Clearly, the pulse peak in the slow medium is delayed by the amount of $0.1133(\mathrm{ps})$ with respect to the vacuum channel.

$$
f(t)=u(t) e^{-\left(\frac{t-t_{0}}{T}\right)^{2}} \sin \left(\omega_{c} t\right) .
$$

Where $u(t)$ is a unit step function with a zero value for all time instants $t<0$ to enforce causality. The field is calculated using the finite-difference time-domain method (FDTD) 11 by solving Maxwell's equations at discrete points in space and time. In this analysis, the discrete time steps are separated by $\Delta t=4 \times 10^{-18}$ (sec) and the space steps are $\Delta z=4 \times 10^{-9}(\mathrm{~m})$. The output response of the medium is plotted in Fig. 6.4 and compared with propagation in vacuum (with the same length $L=30(\mu m)$ ). Figure 6.4 depicts the delay experienced by the Gaussian pulse propagating in the slow light medium as compared to a companion pulse in vacuum. The subluminal pulse peak is retarded with a duration equal to $\sim 0.1133$ (ps) in agreement with Fig. 6.3.

Thus far, we have presented a scenario in which a causal Gaussian pulse propagates in a medium while maintaining subluminal group velocity $v_{g}$. In the next section, we illustrate the behavior of the pulse discontinuities in such a medium.

\footnotetext{
${ }^{1} \mathrm{~A}$ detailed derivation of the update equations used in the FDTD analysis can be found in Appendix A
} 


\subsection{Propagation of Discontinuities in Causal Slow-light Medium}

In this section, we investigate the speed of discontinuities in causal slow light media. The input excitation is turned on for a duration that is roughly equal to $\sim 3 T(0.36(p s))$ before a sudden transitional turn-off of the signal (discontinuity) is introduced at the position of the pulse peak. The responses of both the slow light and vacuum channels (of equal length $L=30(\mu m)$ ) are plotted in Fig. 6.5. Moreover, in both cases of vacuum and slow light channels - excited by the truncated input - the output response is plotted and compared with the corresponding output response if the input pulse had not been terminated.

There are a few interesting observations in regard to Fig. 6.5.

First, it is shown that the sharp termination — which is mathematically represented as a point of non-analyticity (singularity) due to the sudden turn-off of the input-propagates with a strictly luminal speed c. This point and the points immediately following it contain high frequency components which behave toward the medium as if the medium is a vacuum channel. Accordingly, the singularities arrive at the output after duration that is equal to $t=L / c$. This occurs in both the case of propagation in the slow medium (depicted in Fig. 6.5(a)) and in the vacuum channel (as in Fig. 6.5(b)).

Second, although the luminal truncation point (singularity) reaches the output sooner than part of the subluminal energy content of the pulse; nevertheless, this singularity is followed by useful energy content. The duration of this useful energy content-which coincides with the actual response of the non truncated input pulse - roughly extends to the delay duration $\Delta t=\tau_{g}-L / c \sim 0.1133$ (ps) ( $\tau_{g}$ is the subluminal group delay). This implies that for the slow light, the medium is capable of reproducing the input excitation at least for a period $\Delta t \sim 0.1133(p s)$-as if the singularity did not exist. Although one might think that the medium would no longer have a meaningful response at the output after the termination point has occurred; however, this is not true as the medium keeps reacting even past the arrival of the singularity. Of course, this is the consequence of the medium reacting to the portion of the input pulse preceding the singularity. The remainder of the output energy following the singularity is coherent, conserved and can be distinguished without distortion for an amount of $\Delta t \sim 0.1133$ (ps). Finally, after 

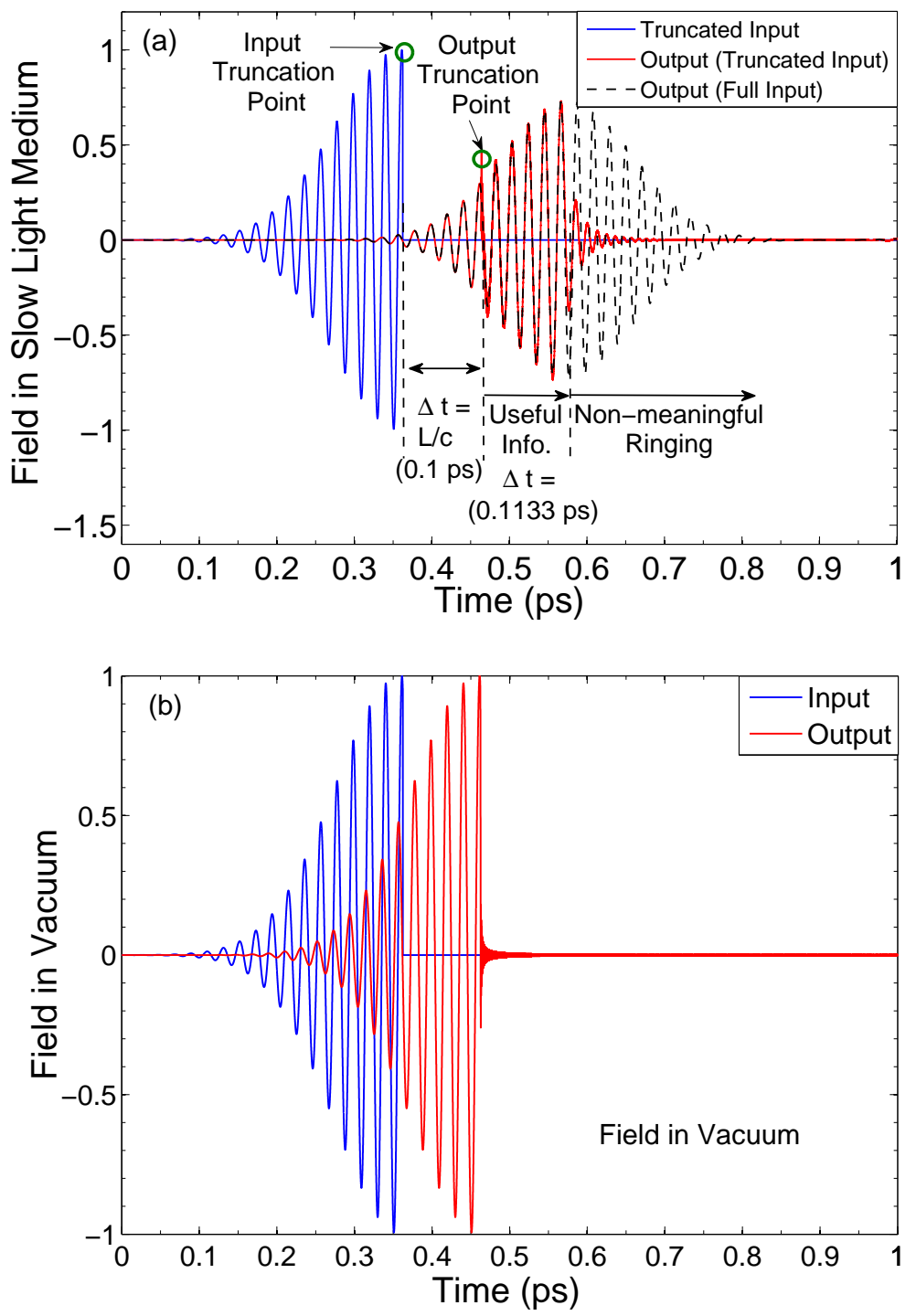

Figure 6.5: Output response of the medium when excited by a truncated pulse. (a) After propagation in a slow light medium of a distance $30(\mu \mathrm{m})$. (b)After propagation in a vacuum channel with the same length.

$\Delta t \sim 0.1133(p s)$, the output pulse starts ringing and has no resemblance to the response of the non truncated input.

Third, for the case of vacuum, the output response represents a mere phase shift of the input pulse in space and time. This is evident in the truncation observed at the output as a response to the input termination.

The above observations can be interpreted as follows: a window which has two boundaries is created; the first boundary is the discontinuity at the front due to the transitional 
turn-on of the pulse, and the other boundary is the second discontinuity as a result of the pulse peak termination. This window maintains a fixed duration $(\sim 3 T)$ during propagation and propagates strictly at $c$. At a propagation distance equal to $30(\mu \mathrm{m})$, the subluminal pulse envelope is retarded (with delay $\Delta t \sim 0.1133(\mathrm{ps})$ with respect to the discontinuity window. However, the window (bounded by the two discontinuities) is aligned with its counterpart in the vacuum channel (depicted in Fig. 6.5(b). In order to study the relation between the arrival of pulse discontinuities as opposed to the arrival of detectable information content 2 of the pulse, the signal-to-noise ratio $(S N R(t))$ is calculated at the detector output.

\subsection{Arrival of Pulse Discontinuities and Detectable Information in Slow-Light Media}

In order to study the behavior of points of non-analyticity of a causal pulse in relation to its detectable information content, we recall the approach carried out in Chapter 5. The $S N R(t)$ is calculated for the pulse considered in Fig. 6.5(a) at a propagation distance $L=30(\mu m)$ and depicted in Fig. 6.6(a).

First, from the $S N R$ analysis it could be observed that the front in both pulses (in the slow medium and vacuum) occur at a strictly luminal speed $(L / c)$ but at a very low value for $S N R$ (-250 dB). Second, for the case of slow light, there is a sudden boost in the $S N R$ which takes place with the arrival of the second precursor (Brillouin precursor). If we set the detectable information to be defined by the time instant when the $S N R$ exceeds the $0-\mathrm{dB}$ level, then it is clear that detectable information is localized within the window of discontinuities (truncation occurs at 0.463 ( $\mathrm{ps}$ ) while detectable information occurs at $0.38(p s))$ consistent with the behavior of detectable information in the fast light case. The corresponding fields, after propagating distance $L=30(\mu m)$ are plotted in Fig. 6.6(b).

On the other hand, if we consider the case of longer propagation distances ( $L=70$ $(\mu m)$ ), unlike the case of fast light, the detectable information is not localized within the window of discontinuities as depicted in Fig. 6.7(a). The $S N R(t)$ analysis shows the

\footnotetext{
${ }^{2}$ Recall from the introduction part that: in some interpretations of information, the pulse singularities (front and termination) have been associated with genuine information.
} 

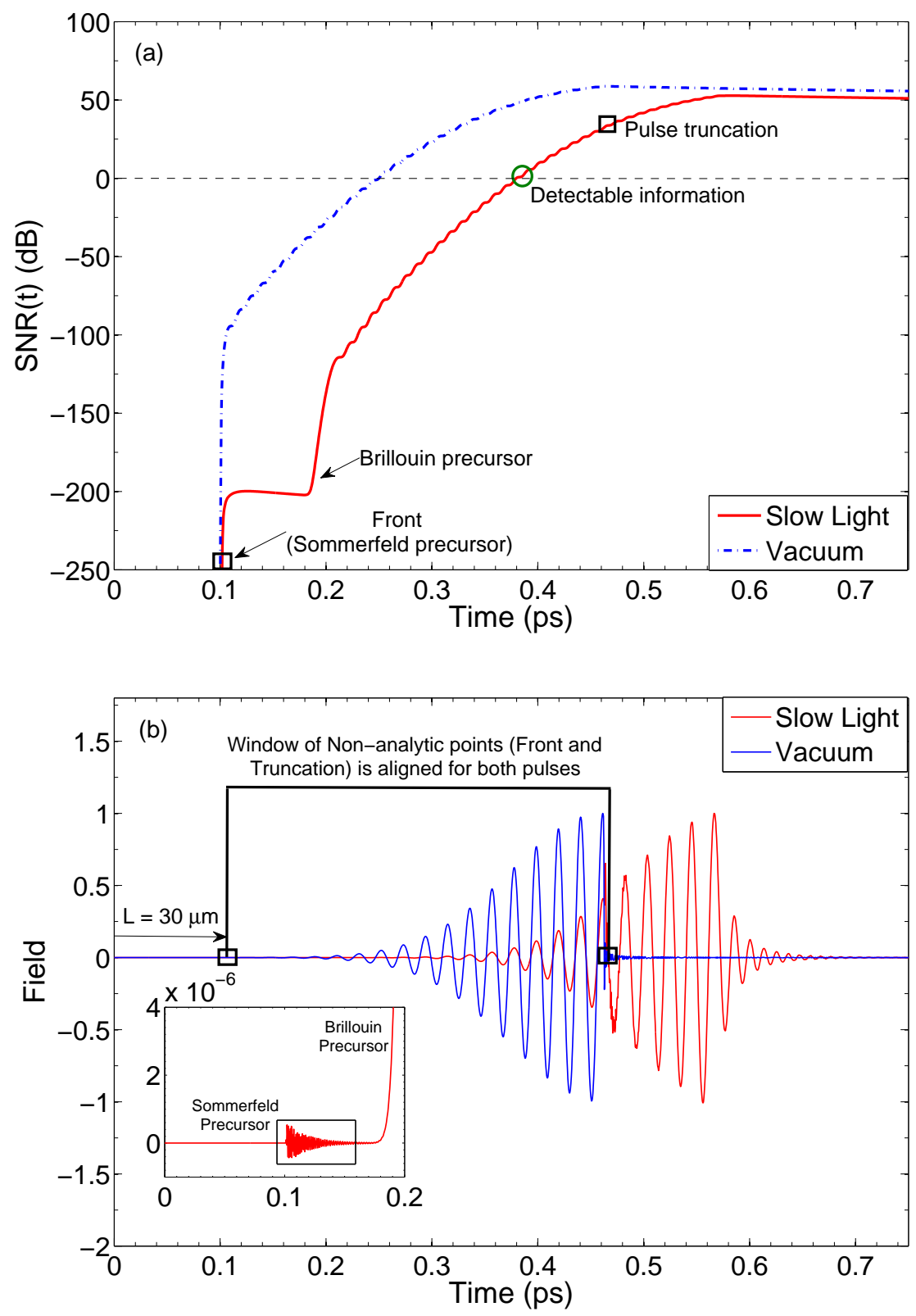

Figure 6.6: (a)Arrival of the detectable information within the window of discontinuities. (b)The strictly luminal behavior of the discontinuities (front and pulse truncation) in the slow-light medium and vacuum.

arrival of the front in vacuum and slow light simultaneously at a very low $S N R$ level. Afterwards, the Brillouin precursor shows up in the case of slow light. It is found out that the detectable information lies outside the window of discontinuities (pulse termination occurs at $0.59(p s)$ while the detectable information occurs at $0.68(p s))$. This can be attributed to the fact that the window of discontinuities is much more time advanced with respect to the center of mass (energy) of the pulse, as depicted in Fig. 6.7(b). 

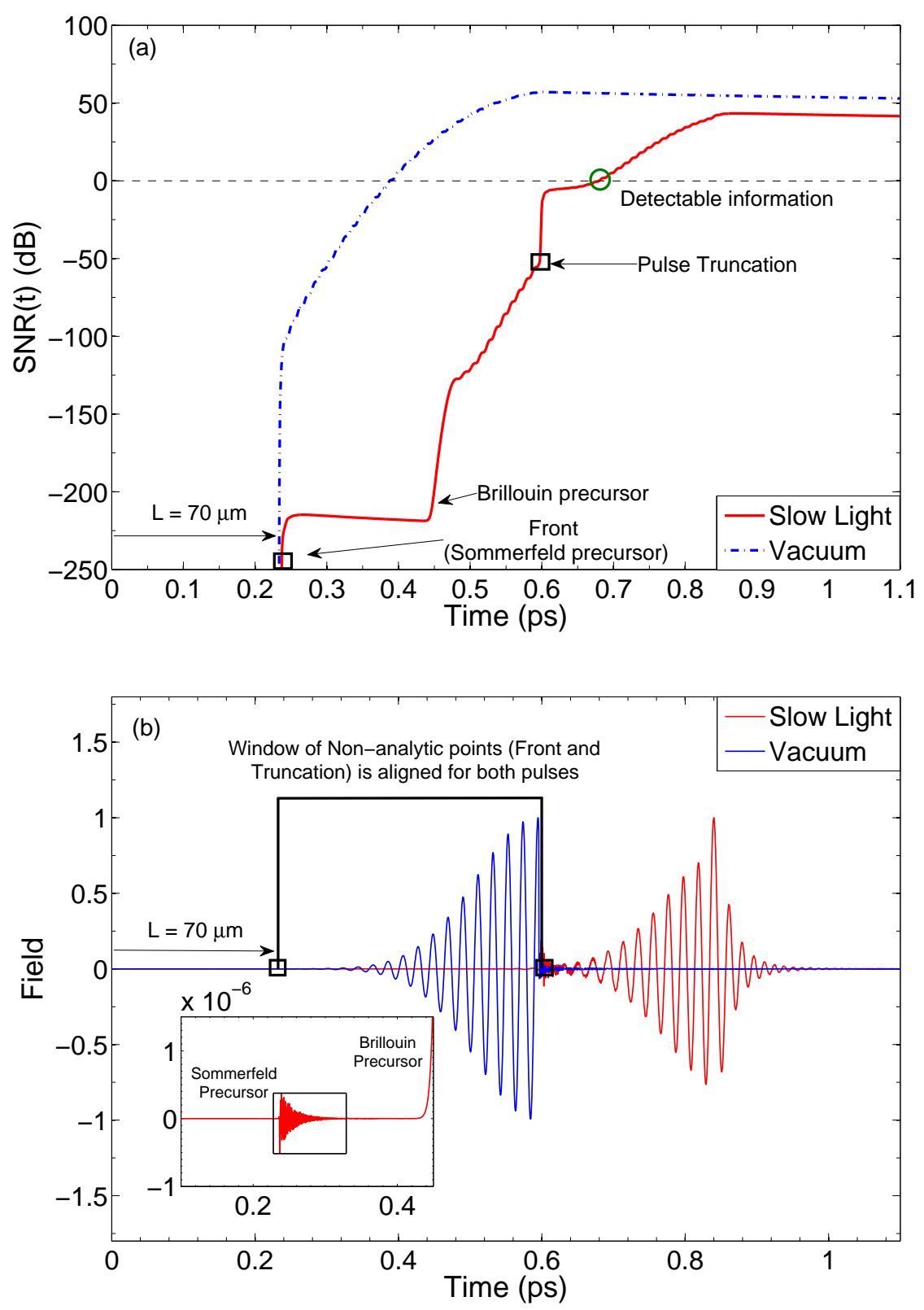

Figure 6.7: (a)Arrival of the detectable information within the window of discontinuities. (b)The strictly luminal behavior of the discontinuities (front and pulse truncation) in the slow-light medium and vacuum.

The above observations suggest that, unlike the case of fast light propagation, the detectable information content of a subluminal pulse can still be retrieved even after the arrival of the pulse discontinuities. This implies that detectable information (signal velocity) cannot be entirely associated with the pulse singularities. 
In this section, the distinction between the behavior of the pulse discontinuities and the corresponding detectable information has been illustrated while considering slow light media. In the next section, we study the behavior of the pulse discontinuities in media that support superluminal propagation to confirm previous predictions.

\subsection{Propagation of Information in Causal Superluminal Medium}

For a medium that exhibits distortion-less superluminal propagation, we consider a double resonance Lorenztian medium with gain. The refractive index of such medium is expressed as:

$$
n(\omega)=\sqrt{1+\frac{\omega_{p, 1}^{2}}{\omega^{2}-\omega_{0,1}^{2}+2 i \delta \omega}+\frac{\omega_{p, 2}^{2}}{\omega^{2}-\omega_{0,2}^{2}+2 i \delta \omega}} .
$$

Where, $\omega_{p, j}$ and $\omega_{0, j}(j=1$ or 2$)$ are the plasma and resonance frequencies associated with the first and second resonances, and $\delta$ is the phenomenological line-width. The group delay of the gain medium, as a function of detuning is depicted in Fig. 6.8(a).

Clearly, the selected medium exhibits superluminal propagation in the range of frequencies around $\omega=5 \times 10^{15}\left(\mathrm{sec}^{-1}\right)$ (where $L / c-\left(\tau_{g}\right)_{\text {sup }} \sim 1(\mathrm{fsec})$ ). As such, we chose an input Gaussian excitation centered at $\omega_{c}=5 \times 10^{15}\left(\mathrm{sec}^{-1}\right)$ and pulse width $2 T$ $=8(\mathrm{fsec})$. The output response of the medium is computed using FDTD method with discrete time steps $\Delta t=2 \times 10^{-18}(\mathrm{sec})$ and space steps $1.25 \times 10^{-9}(\mathrm{~m})$. This choice of parameters ensures computational stability for the gain medium at the specified carrier frequency at long propagation distances.

The output response of the medium, after propagating for $2.5(\mu \mathrm{m})$ is plotted in Fig. 6.8(b) and compared with the case of vacuum. It is shown that the superluminal pulse exhibits slight compression and peak advanced equal to $\sim 1(f s)$.

In order to investigate the causal connection between the input and output pulses, we introduce a sudden turn-off in the input Gaussian pulse at the position of its peaksimilar to the approach carried on in section 6.3-and observe the output response for such excitation. This approach is depicted in Fig. 6.9 (a) for the case of a superluminal pulse and is applied for the case of propagation in a vacuum channel (as illustrated in Fig. 6.9(b)) for comparison.

The results obtained in both figures can be summarized as follows: 

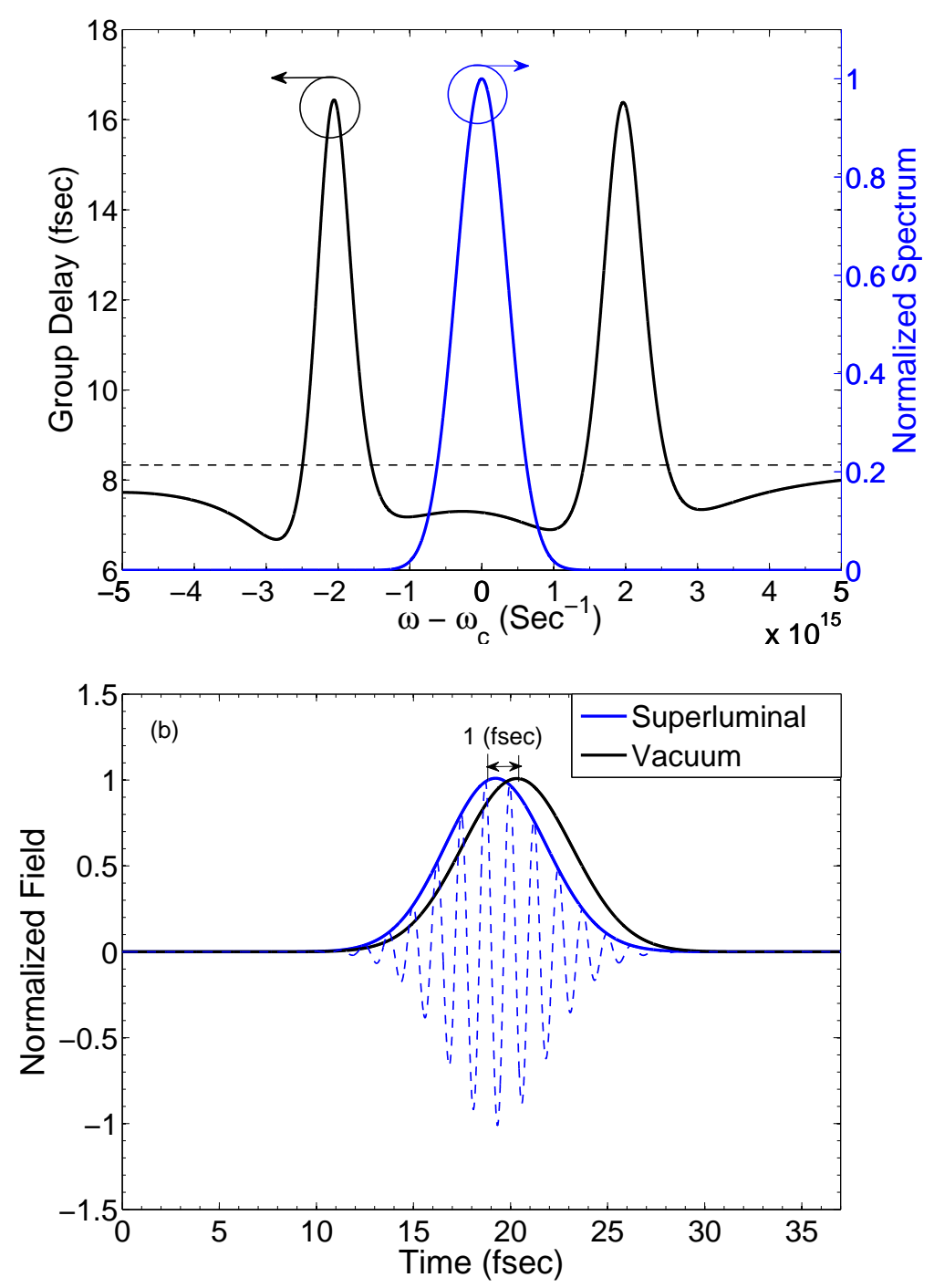

Figure 6.8: The superluminal medium response in the frequency and time domains. (a)Input pulse spectrum fitted over the group delay plot (as a function of frequency) of a superluminal medium. The black dashed lines correspond to group delay in vacuum. (b)Comparison of the time domain response of the superluminal medium and a vacuum channel after propagation distance $L=2.5(\mu \mathrm{m})$.

First, it is observed that the discontinuity - which corresponds to the transitional termination of the input pulse - arrives at the output after duration that is strictly luminal and equal to $L / c$ despite the type of the medium (whether it is superluminal medium or vacuum).

Second, for propagation in a vacuum channel - in agreement with the discussion in section 6.4 - the output pulse is truncated at the same position of the input truncation. In this case, it is clear that the input and output pulse peaks are causally connected.

Third, more interestingly, when introducing a transitional turn-off for the input pulse 

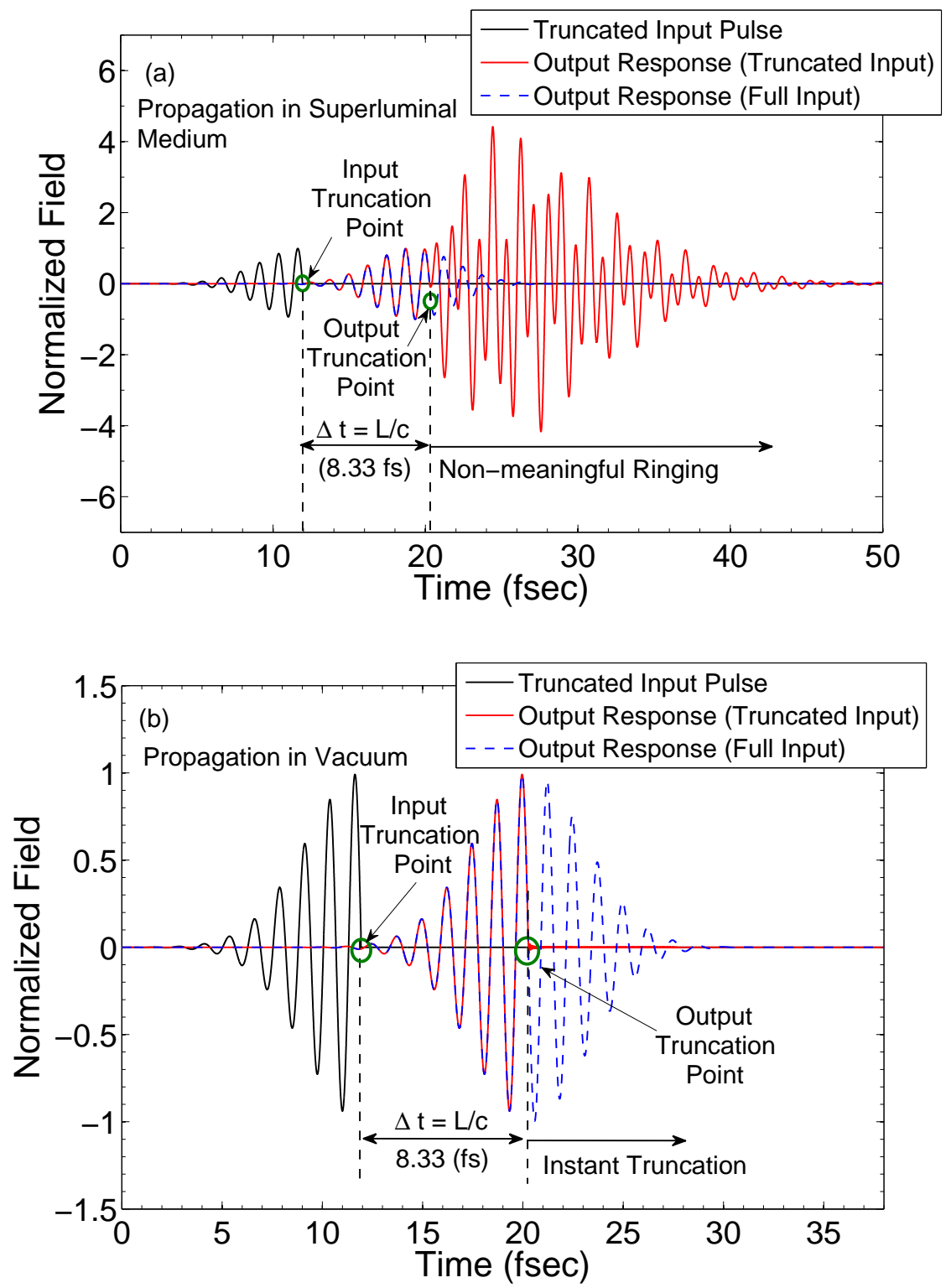

Figure 6.9: The input spectrum fitted over the group delay plot for the case of a superluminal medium. The black dashed lines correspond to group delay in vacuum.

in the superluminal medium, the remainder of the energy content past the output singularity conveys non-meaningful ringing (unlike a subluminal pulse). The energy transferred from the (gain) medium to the field (at the point of truncation) does not convey additional useful information about the input pulse. This implies that all the input information that interacted with the medium before the termination point, has already evolved at the output before the truncation instant. These observations are in full agreement with 63, 64, 65, 66]. 
The above observations imply that detectable information (in the case of fast light media) is always localized within the discontinuity window, unlike the case of slow light propagation in which detectable information is not necessarily localized within this window.

\subsection{Conclusion}

From a fundamental point-of-view, we investigated the transfer of information content for causal Gaussian pulses in dispersive media supporting slow and fast light propagation. Using truncated optical pulses at the input of dispersive media, it has been shown that a pulse can carry multiple discontinuities and the information content of the pulse remains below the detection threshold (not practically detectable) even after the arrival of all the non-analytic points of the pulse at the receiver. We thus argue that, from a practical point-of-view, information can not be entirely associated with the pulse discontinuities. The distinction between the evolution of pulse discontinuities and the detectable information content of the pulse has been demonstrated. This analysis highlights the capabilities and limitations of slow light and fast light applications. 


\section{Chapter 7}

\section{Conclusion and Future Work}

In this thesis, the evolution of energy - together with the associated information contentof a causal Gaussian pulse propagating in a dispersive medium has been investigated. The analysis focused on the interesting phenomena of fast (superluminal) and slow (subluminal) light propagation. Using the saddle point analysis (the method of steepest descent) the results of which have been validated using the finite-difference time-domain methodthe fundamental constraints on superluminal propagation have been highlighted in light of factors such as: the medium length, input pulse width and dispersion strength, as presented in Chapter 4. Moreover, a closed form analytic expression has been derived to predict the superluminal-to-subluminal cutoff distance in a double resonance gain medium. Such expression becomes useful when designing any experimental setup that utilizes superluminal propagation.

In Chapter 5, a systematic approach to quantify the velocity of detectable information of a superluminal Gaussian pulse in an inverted medium, has been presented. The analysis accounted for the medium generated noise in addition to the noise associated with the detection process. It has been shown that superluminal propagation can be useful to reduce signal detection latency in communication systems that are limited by the detector noise. Cases in which the velocity of detectable information can exceed its counterpart in a vacuum channel of the same length - without violating Einstein causality - have been presented together with their interpretation. Interestingly, it has been shown that Einstein causality is always preserved without the need to refer to the practically undetectable front (or precursors). 
Finally, the dynamical exchange of information and energy in causal dielectrics has been discussed in Chapter 6. Using truncated pulses at the input of a slow light (and a fast light) medium, it has been shown that the energy exchange between the optical pulse and a passive slow light medium involves storage and full recovery of the input information past the truncation point. On the other hand, energy exchange in active superluminal media does not involve storage of useful information and later recovery past the termination point. We presented scenarios in which a pulse can carry multiple discontinuities while the information content of the pulse remains below the detection threshold (not practically detectable) even after the arrival of all the non-analytic points of the pulse at the receiver. This analysis thus shows that relating information with the points of non-analyticity (discontinuities) of a pulse is not a conclusive approach.

In the following subsections, we propose possible extensions to the present analysis to be taken into account in future.

\subsection{Future work}

\subsubsection{Experimental demonstration of superluminal pulse transition to a sub- luminal regime}

The scope of this thesis has been limited to the theoretical analysis of pulse propagation in a causal dispersive medium. One possible extension to this analysis is to further implement some of the discussed concepts in practice. For instance, in Chapter 3, the fundamental limitations imposed by the medium length on superluminal propagation can be experimentally tested. The parameters of the double resonance Lorentzian medium in Chapter 3 have been selected so that it correspond to a practical inverted medium that can be easily realized using ammonia vapor cells at $780(\mathrm{~nm})$. The detailed analysis of this medium can be found in ref [21]. Accordingly, by carrying out the experiment at the four different propagation distances - as specified in the theoretical analysis in Chapter 5 - one can record the transition of a superluminal Gaussian pulse to the subluminal regime at longer propagation distance, and thus validate the analytic closed form expression of Eq. 4.4, experimentally. 


\subsubsection{The role of the detector in information velocity}

In Chapter 5, it has been shown that - depending on the interplay between the detector noise with respect to the medium generated noise - an inverted medium can be useful in reducing the signal detection latency as compared to propagation in a vacuum channel. One possible extension to this work is to further investigate the role played by the type of detection. For instance, instead of using a photo-multiplier detector - as has been adopted in Chapter 4-one can study the effect of employing other detection schemes such as heterodyne detection.

\subsubsection{Utilizing the information capacity of superluminal media using ad- vanced modulation techniques}

This thesis tackled the following question: "Can superluminal propagation in a noisy dispersive inverted medium be used to achieve faster information transfer?". The respective analysis has been presented in Chapter 5; in which it was found that it is indeed possible to reduce the latency (transfer time) of detectable information under some constraints on a) the channel (medium) length. b) The channel dispersion strength (and associated noise). c) The detector noise. Nevertheless, the problem still involves other complexities that have not been resolved yet. For instance, in our analysis, we considered a simple on-off keying communication scheme. Under this on-off keying protocol, a superluminal channel carries merely a single bit of information in each transmission. While this analysis is useful to explore the potential of superluminal propagation; however, it does not uncover the full potential as well as other challenges (and limitations) of superluminal channels. One possible extension to this work is to give more attention to the signal generation stage in the form of deploying more complex modulation schemes.

One can investigate the possibility of packing more information in a superluminal channel using advanced modulation techniques that are customized with respect to the dispersive channel behavior. The objective of such study would be to utilize superluminal channels at their full information capacity, assess the practical potential of using these optimized channels in optical interconnects (for time-sensitive applications), and to investigate the (new) challenges that may arise due to the additional degree of freedom (at the signal generation stage). One way to perform this is to encode information in 
the input pulse using M-ary (multi) phase-shift keying and study its propagation in a superluminal dispersive medium with phase-sensitive gain. In such case, the information content carried by different symbols (input pulses) would experience different channelgenerated noise during propagation. By carefully designing the dispersion characteristics of the medium (which affects its noise characteristics), we can obtain a scenario in which one symbol is transmitted with both superluminal delay and minimum amount of noise, simultaneously; as the extra noise will be 'dumped' into the information content (phase) of another symbol. In such case, it is interesting to investigate the speed of information in this clean symbol, to evaluate the gross throughput (successful transmission of information by all symbols) as compared with a normal dispersion channel, and to determine the maximum number of symbols that can be reliably supported (packed) on the channel. In order to utilize the superluminal channel at its full capacity, some optimization techniques will be applied: first, the input pulses will be transmitted in an asymmetric manner (i.e. less noisy symbols will be transmitted more frequently); this involves designing a suitable source code so that more information content is assigned to the clean symbols. Second, the channel noise can be smartly controlled (by dynamically tuning the medium dispersion strength) so that each pulse is transmitted with minimum amount of noise affecting its encoded information. The outcome of this study would be an experimental demonstration of a full communication system with source code (dictionary) that is transmitted on an optimized superluminal channel with minimum delay and maximum throughput. The results would have a huge impact on the design of interconnects and buses that require efficient transmission of many symbols in parallel; where latency reduction in each symbol would lead to remarkable latency reduction across the entire bus. 


\section{Chapter 8}

\section{Refereed Publications}

\subsection{Articles published in refereed journals:}

1. Ahmed H. Dorrah, and Mo Mojahedi, "Velocity of detectable information in fasterthan-light pulses," Physical Review A, Vol. 90, 033822, 2014.

[DOI: http://dx.doi.org/10.1103/PhysRevA.90.033822].

2. Ahmed H. Dorrah, Levent Kayili, and Mo Mojahedi, "Superluminal propagation and information transfer: a statistical approach in the microwave domain," Physics Letters A, Vol. 378, Issue 44, pp. 3218-3224, 2014.

[DOI: http://dx.doi.org/10.1016/j.physleta.2014.09.043].

\subsection{Articles presented in refereed conferences:}

1. Ahmed H. Dorrah, and Mo Mojahedi, "Can superluminal propagation in a noisy dispersive medium reduce signal detection latency?," IEEE Photonics Conference, San Diego, USA (International conference) - Oral presentation, October 16th, 2014. (Paper ID: 325811).

2. Ahmed H. Dorrah, and Mo Mojahedi, "Dynamical evolution of information and energy in causal dispersive media," IEEE Photonics Conference, San Diego, USA (International conference) - Oral presentation, October 15th, 2014.(Paper ID: 325797). 
Appendices 


\section{Appendix A}

\section{FDTD Update Equations}

In this section, the update equations needed for the finite-difference time-domain (FDTD) calculations - incorporated in Chapter 3 and Chapter 6-are derived. The analysis can be utilized for the case of a single resonance as well as a double resonance Lorentziam medium.

Starting from Ampere's and Faraday's pair of equations,

$$
\nabla \times E=-\mu \frac{d H}{d t}
$$

and

$$
\nabla \times H=\frac{d D}{d t},
$$

and given the fact that - for a temporally dispersive Lorentzian medium with a double resonance - the permittivity $\epsilon(\omega)$ is expressed as:

$$
\epsilon(\omega)=\epsilon_{0}-\frac{\epsilon_{0} \omega_{p}^{2}}{\omega_{0}^{2}-\omega^{2}-2 \delta \omega i}-\frac{\epsilon_{0} \omega_{p 2}^{2}}{\omega_{02}^{2}-\omega^{2}-2 \delta \omega i},
$$

consequently, Eq A.2 can be written as

$$
\nabla \times H=-i \omega \epsilon_{0} E-i \omega Q_{1}-i \omega Q_{2}
$$

where,

$$
\begin{aligned}
Q_{1} & =\frac{\epsilon_{0} \omega_{p}^{2}}{\omega_{0}^{2}-\omega^{2}-2 \delta \omega i} . \\
Q_{2} & =\frac{\epsilon_{0} \omega_{p 2}^{2}}{\omega_{02}^{2}-\omega^{2}-2 \delta \omega i} .
\end{aligned}
$$


Accordingly, each resonance $(\mathrm{k})$ can be associated with an intermediate variable $Q_{k}$ and can be expressed as follows

$$
Q_{k} \omega_{0, k}^{2}-\omega^{2} Q_{k}-i \omega \delta Q_{k}=\epsilon_{0} \omega_{p, k}^{2} E
$$

where $(\mathrm{k}=1$ or 2$)$ for a double resonance Lorentzian medium. For a single harmonic with time dependence $e^{-i \omega t}$, the term $(-i \omega)$ is equivalent to performing the time derivative $\frac{d}{d t}$. Likewise, $\omega^{2}$ is replaceable with $\frac{d^{2}}{d t^{2}}$. Therefore, Eq A.7 can be rewritten as

$$
Q_{k} \omega_{0, k}^{2}+\frac{d^{2} Q_{k}}{d t^{2}}+\frac{d Q_{k}}{d t} \delta=\epsilon_{0} \omega_{p, k}^{2} E
$$

Using finite-difference approximation to discretize the time derivatives in Eq A.8, we obtain

$$
Q_{k} \omega_{0, k}^{2}+\frac{Q_{k}^{n+1}-2 Q_{k}^{n}+Q_{k}^{n-1}}{d t^{2}}+\frac{Q_{k}^{n+1}-Q_{k}^{n-1}}{2 d t} \delta=\epsilon_{0} \omega_{p, k}^{2} E^{n}
$$

Thus by grouping the coefficients of $Q_{k}^{n+1}, Q_{k}^{n}$ and $Q_{k}^{n-1}$, the update equation for $Q_{k}^{n+1}$ can be written as

$$
Q_{k}^{n+1}=\left[\frac{\delta d t-2}{\delta d t+2}\right] Q_{k}^{n-1}+2\left[\frac{2-\omega_{0, k}^{2} d t^{2}}{\delta d t+2}\right] Q_{k}^{n}+2\left[\frac{2 \epsilon_{0} \omega_{p, k}^{2} d t^{2}}{\delta d t+2}\right] E^{n} .
$$

As for the update equations of the electric field $E, \mathrm{Eq}$ A.4 can be rewritten in the following form

$$
\nabla \times H^{n+1 / 2}=\epsilon_{0} \frac{d E}{d t}+\sum_{k=1}^{n} \frac{d Q_{k}}{d t}
$$

where $n$ denotes the number of resonances in the Lorentzian medium.

The time derivatives in Eq A.11 can now be replaced with a discrete forward finite difference. In this case, Eq A.11 can be expressed as

$$
\nabla \times H^{n+1 / 2}=\epsilon_{0} \frac{E^{n+1}-E^{n}}{d t}+\sum_{k=1}^{n} \frac{Q_{k}^{n+1}-Q_{k}^{n}}{d t}
$$

Accordingly, the update equation for $E^{n+1}$ is written as

$$
E^{n+1}=E^{n}+\frac{d t}{\epsilon_{0}}\left(\Delta \times H^{n+1 / 2}\right)-\frac{1}{\epsilon_{0}} \sum_{k=1}^{n}\left(Q_{k}^{n+1}-Q_{k}^{n}\right) .
$$

By replacing the curl in Eq A.13 with its finite difference representation, we obtain 


$$
E^{n+1}=E^{n}+\frac{d t}{\epsilon_{0}}\left[\frac{H_{x+1}^{n+1 / 2}-H_{x}^{n+1 / 2}}{d x}\right]-\frac{1}{\epsilon_{0}} \sum_{k=1}^{n}\left(Q_{k}^{n+1}-Q_{k}^{n}\right) .
$$

Finally, the update equation for the magnetic field $H$ can be derived in a straight forward manner from $\mathrm{Eq}$ A.1

$$
H^{n+1 / 2}=H^{n-1 / 2}-\frac{d t}{\mu}\left[\frac{E_{x+1 / 2}^{n}-E_{x-1 / 2}^{n}}{d x}\right]
$$

In summary, the update equations for the electric field $E$, magnetic field $H$ have been derived for the case of temporally dispersive Lorentzian media with an arbitrary number of resonances. The above equations are also valid for the case of passive as well as active media. The field calculations at each discrete point in space and time can be carried out in the following sequence: first, the magnetic field is calculated from Eq A.15, then the intermediate auxiliary variables $\left(Q_{k}\right)$ are calculated from Eq A.10. Finally, the magnetic field and the auxiliary variables are substituted in Eq A.14 in order to calculate the electric field. 


\section{Appendix B}

\section{Derivation of}

\section{Superluminal-to-Subluminal Cutoff}

\section{Distance}

In this section, the equation for the cut-off length of a double resonance Lorenzian gain medium at which a superluminal Gaussian pulse becomes superluminal is derived. For a typical double resonance Lorentzian medium with gain, the maximum medium gain (which is typically associated with the subluminal region) occurs at the resonance frequencies $\left(\omega_{0_{i}}\right)$. On the other hand, the gain encountered within the superluminal region (which is located between the gain doublet) is minimal. Accordingly, the spectral components comprising the input excitation are scaled differently depending on whether they are located within the superluminal region or not. At a specific length (cut-off distance), the superluminal pulse becomes dominated by its subluminal components. Therefore, by calculating the energy content of the subluminal part and comparing it with the superluminal energy content of the pulse and performing the formulation as a function of the medium length, one can derive an expression for the cut-off distance at which the superluminal and subluminal energy components are equal. The double resonance gain medium considered in the analysis is given by:

$$
n(\omega)=\sqrt{1+\frac{\omega_{p, 1}^{2}}{2 i \delta \omega+\omega^{2}-\omega_{0,1}^{2}}+\frac{\omega_{p, 2}^{2}}{2 i \delta \omega+\omega^{2}-\omega_{0,2}^{2}}} .
$$


For this case, the approximation $(\sqrt{1+x} \approx 1+x / 2)$ holds and thus $n(\omega)$ can be re-written as follows:

$$
n(\omega)=1+\frac{1}{2}\left(\frac{\omega_{p, 1}^{2}}{2 i \delta \omega+\omega^{2}-\omega_{0,1}^{2}}+\frac{\omega_{p, 2}^{2}}{2 i \delta \omega+\omega^{2}-\omega_{0,2}^{2}}\right) .
$$

Accordingly, the real part of $n(\omega)$ is given by:

$$
n_{r}(\omega)=\frac{1}{2}\left[\frac{\left(\omega^{2}-\omega_{0_{1}}^{2}\right) \omega_{p, 1}^{2}}{4 \delta^{2} \omega^{2}+\omega^{4}-2 \omega_{0,1}^{2} \omega^{2}+\omega_{0,1}^{4}}+\frac{\left(\omega^{2}-\omega_{0_{2}}^{2}\right) \omega_{p, 2}^{2}}{4 \delta^{2} \omega^{2}+\omega^{4}-2 \omega_{0,2}^{2} \omega^{2}+\omega_{0,2}^{4}}+2\right] .
$$

Likewise, the imaginary part of $n(\omega)$ is written as:

$$
n_{i}(\omega)=-i \delta \omega\left[\frac{\omega_{p, 1}^{2}}{4 \delta^{2} \omega^{2}+\left(\omega^{2}-\omega_{0,1}^{2}\right)^{2}}+\frac{\omega_{p, 2}^{2}}{4 \delta^{2} \omega^{2}+\left(\omega^{2}-\omega_{0,2}^{2}\right)^{2}}\right] .
$$

Furthermore, the medium gain is expressed as a function of $\omega, z$, and $n(\omega)$ by $g(\omega)=e^{-\omega \operatorname{Im}\{n(\omega)\} z / c}$. In order to derive the ratio between the gain associated with the subluminal part as compared to the superluminal, we calculate the medium gain in the corresponding frequency regions. For the subluminal region, the maximum gain typically occurs at the position of the resonance frequency $\left(\omega_{0_{i}}\right)$; as such, the imaginary component of $n(\omega)$ evaluated at $\left(\omega_{0,1}\right)$ is given by:

$$
\operatorname{Im}\left\{n\left(\omega_{0,1}\right)\right\}=-\frac{\delta \omega_{0,1} \omega_{p, 2}^{2}}{4 \delta^{2} \omega_{0,1}^{2}+\left(\omega_{0,1}^{2}-\omega_{0,2}^{2}\right)^{2}}-\frac{\omega_{p, 1}^{2}}{4 \delta \omega_{0,1}}
$$

Similarly,

$$
\operatorname{Im}\left\{n\left(\omega_{0,2}\right)\right\}=-\frac{\delta \omega_{0,2} \omega_{p, 1}^{2}}{4 \delta^{2} \omega_{0,2}^{2}+\left(\omega_{0,1}^{2}-\omega_{0,2}^{2}\right)^{2}}-\frac{\omega_{p, 2}^{2}}{4 \delta \omega_{0,2}}
$$

As for the gain in the superluminal region (which is typically centered between the two resonances), the imaginary component of $n(\omega)$ can be expressed as:

$$
\begin{aligned}
\operatorname{Im}\left\{n\left(\frac{\omega_{0,1}+\omega_{0,2}}{2}\right)\right\}=\frac{1}{2} \delta\left(\omega_{0,1}+\omega_{0,2}\right) \times\{ & -\frac{\omega_{p, 1}^{2}}{\delta^{2}\left(\omega_{0,1}+\omega_{0,2}\right)^{2}+\left[\omega_{0,1}^{2}-\frac{1}{4}\left(\omega_{0,1}+\omega_{0,2}\right)^{2}\right]^{2}} \\
& \left.-\frac{\omega_{p, 2}^{2}}{\delta^{2}\left(\omega_{0,1}+\omega_{0,2}\right)^{2}+\left[\omega_{0,2}^{2}-\frac{1}{4}\left(\omega_{0,1}+\omega_{0,2}\right)^{2}\right]^{2}}\right\} .
\end{aligned}
$$


In order to calculate the gain in the subluminal region, we first evaluate $\omega_{0,1} \times$ $\operatorname{Im}\left\{n\left(\omega_{0,1}\right)\right\}$ (or equivalently $\omega_{0,2} \times \operatorname{Im}\left\{n\left(\omega_{0,2}\right)\right\}$ since the only change between the two expressions is substituting $\omega_{0,1}$ for $\left.\omega_{0,2}\right)$ and simplify the resulting expression to get:

$$
\omega_{0,1} \times \operatorname{Im}\left\{n\left(\omega_{0,1}\right)\right\}=-\frac{\delta \omega_{0,1}^{2} \omega_{p, 2}^{2}}{4 \delta^{2} \omega_{0,1}^{2}+\left(\omega_{0,1}^{2}-\omega_{0,2}^{2}\right)^{2}}-\frac{\omega_{p, 1}^{2}}{4 \delta} .
$$

By replacing $\omega_{0,2}$ with $\omega_{0,1}+\Delta$, and ignoring any higher order non-linear terms in $\Delta$, Eq. B.8 can be simplified to:

$$
\omega_{0,1} \times \operatorname{Im}\left\{n\left(\omega_{0,1}\right)\right\}=-\frac{\omega_{p, 2}^{2}}{4 \delta \Delta^{2}}-\frac{\omega_{p, 1}^{2}}{4 \delta}
$$

However, since usually $\frac{\omega_{p, 2}^{2}}{4 \delta \Delta^{2}}<<\frac{\omega_{p, 1}^{2}}{4 \delta}$, this can be further simplified to:

$$
\omega_{0,1} \times \operatorname{Im}\left\{n\left(\omega_{0,1}\right)\right\}=-\frac{\omega_{p, 1}^{2}}{4 \delta} .
$$

Similarly, we can get:

$$
\omega_{0,2} \times \operatorname{Im}\left\{n\left(\omega_{0,2}\right)\right\}=-\frac{\omega_{p, 2}^{2}}{4 \delta} .
$$

In most practical cases, the values for $\omega_{p, 1}$ and $\omega_{p, 2}$ are very close in order to maintain a flat region between the gain doublets. Furthermore, the frequency detuning between the resonances $\left(\omega_{0,1}\right.$ and $\left.\omega_{0,2}\right)$ is sufficiently close so that we may account for the gain of either resonance in terms of the averaged value for $\omega \operatorname{Im}\{n(\omega)\}$. Accordingly, the following expression may be used instead of Eq. B.10 and B.11 to calculate the gain in the subluminal region:

$$
\omega_{0, i} \times \operatorname{Im}\left\{n\left(\omega_{0, i}\right)\right\}=-\frac{\omega_{p, 2}^{2}+\omega_{p, 1}^{2}}{8 \delta} .
$$

This approximation is valid as long as $\omega_{0,2}$ is not much larger than $\omega_{0,1}\left(\omega_{0,2}<10 \times \omega_{0,1}\right)$ which is typically the case in most practical scenarios. Consequently, the gain associated with the subluminal region (for only one resonance) is expressed by:

$$
g(\omega, z)_{\text {subluminal }}=\exp \left[\left(\frac{\omega_{p, 2}^{2}+\omega_{p, 1}^{2}}{8 \delta}\right) \times \frac{z}{c}\right]
$$


To calculate the gain at the superluminal region, we must first evaluate $\frac{\omega_{0,1}+\omega_{0,2}}{2} \times$ $\operatorname{Im}\left\{n\left(\frac{\omega_{0,1}+\omega_{0,2}}{2}\right)\right\}$ and simplify the resulting expression. Consequently, the following equation can be derived:

$$
\begin{gathered}
\frac{\omega_{0,1}+\omega_{0,2}}{2} \times \operatorname{Im}\left\{n\left(\frac{\omega_{0,1}+\omega_{0,2}}{2}\right)\right\}=\frac{1}{4} \delta\left(\omega_{0,1}+\omega_{0,2}\right)^{2} \times \\
\left\{-\frac{\omega_{p, 1}^{2}}{\delta^{2}\left(\omega_{0,1}+\omega_{0,2}\right)^{2}+\left[\omega_{0,1}^{2}-\frac{1}{4}\left(\omega_{0,1}+\omega_{0,2}\right)^{2}\right]^{2}}-\right. \\
\left.\frac{\omega_{p, 2}^{2}}{\delta^{2}\left(\omega_{0,1}+\omega_{0,2}\right)^{2}+\left[\omega_{0,2}^{2}-\frac{1}{4}\left(\omega_{0,1}+\omega_{0,2}\right)^{2}\right]^{2}}\right\} .
\end{gathered}
$$

In general, $\delta<<\omega_{0, i}$ and hence the term $\delta^{2}\left(\omega_{0,1}+\omega_{0,2}\right)^{2}$ in the denominator is usually $\sim 2$ orders of magnitude smaller than the other term in the denominator and as such can be omitted in both fractions. Accordingly, we get:

$$
\begin{gathered}
\frac{\omega_{0,1}+\omega_{0,2}}{2} \times \operatorname{Im}\left\{n\left(\frac{\omega_{0,1}+\omega_{0,2}}{2}\right)\right\}=\frac{1}{4} \delta\left(\omega_{0,1}+\omega_{0,2}\right)^{2} \times \\
\left\{-\frac{\omega_{p, 1}^{2}}{\left[\omega_{0,1}^{2}-\frac{1}{4}\left(\omega_{0,1}+\omega_{0,2}\right)^{2}\right]^{2}}-\frac{\omega_{p, 2}^{2}}{\left[\omega_{0,2}^{2}-\frac{1}{4}\left(\omega_{0,1}+\omega_{0,2}\right)^{2}\right]^{2}}\right\} .
\end{gathered}
$$

By replacing the term $\omega_{0,2}$ with $\omega_{0,1}+\Delta$, and ignoring any higher order non-linear terms in $\Delta$, Eq. B.15 can be simplified to:

$$
\begin{array}{r}
\frac{\omega_{0,1}+\omega_{0,2}}{2} \times \operatorname{Im}\left\{n\left(\frac{\omega_{0,1}+\omega_{0,2}}{2}\right)\right\}=\frac{1}{4} \delta\left(\omega_{0,1}+\omega_{0,2}\right)^{2} \times \\
{\left[-\frac{\omega_{p, 1}^{2}}{\omega_{0,1}^{2}\left(\omega_{0,2}-\omega_{0,1}\right)^{2}}-\frac{\omega_{p, 2}^{2}}{\omega_{0,2}^{2}\left(\omega_{0,2}-\omega_{0,1}\right)^{2}}\right] .}
\end{array}
$$

Thus the gain at the superluminal region can be written in a straight forward manner in the following form:

$$
g(\omega, z)_{\text {superluminal }}=\exp \left[\frac{1}{4} \delta\left(\omega_{0,1}+\omega_{0,2}\right)^{2}\left(\frac{\omega_{p, 1}^{2}}{\omega_{0,1}^{2}\left(\omega_{0,2}-\omega_{0,1}\right)^{2}}+\frac{\omega_{p, 2}^{2}}{\omega_{0,2}^{2}\left(\omega_{0,2}-\omega_{0,1}\right)^{2}}\right) \frac{z}{c}\right]
$$

Thus far, we have derived expressions for the gain associated with the superluminal and subluminal regions. In the following, we include the input pulse into the calculations in order to evaluate the corresponding energy content in the superluminal and the sublu- 
minal regions. Accordingly, the cut-off distance (at which the energy contents level out) can be derived. We chose an input modulated Gaussian excitation with carrier frequency $\left(\omega_{c}\right)$ that is centered at $\left(\frac{\omega_{0,1}+\omega_{0,2}}{2}\right)$. The input pulse envelope is given by $e^{\left(-\frac{1}{4} T^{2}\left(\omega-\omega_{c}\right)^{2}\right)}$. We estimate the subluminal region associated with the resonances $\left(\omega_{0,1}\right.$ and $\left.\omega_{0,2}\right)$ to be extended over the frequency ranges: $\left[\omega_{0,1}-3 \delta, \omega_{0,1}+3 \delta\right]$ and $\left[\omega_{0,2}-3 \delta, \omega_{0,2}+3 \delta\right]$ respectively. Consequently, the energy content of the input pulse that lies within the subluminal part (for each resonance) can be roughly given by:

$$
6 \delta e^{\left(-\frac{1}{4} T^{2}\left(\omega_{0,1}-\omega_{c}\right)^{2}\right)} .
$$

To account for both resonances, Eq. B.18 is re-written as:

$$
12 \delta e^{\left(-\frac{1}{4} T^{2}\left(\omega_{0,1}-\omega_{c}\right)^{2}\right)} .
$$

The gain experienced by this component has been given in Eq. B.13.

As for the superluminal region, we assume that it extends over the remaining frequency range. The average energy content of the input pulse that lies within the superluminal region is thus given by:

$$
\frac{1}{2}\left(-6 \delta-\omega_{0,1}+\omega_{0,2}\right),
$$

where the factor $\left(\frac{1}{2}\right)$ accounts for averaging the peak value of the Gaussian (centered at the carrier frequency) over the superluminal frequency range. The gain experienced by this energy component has been given in Eq. B.17.

In order to obtain the cut-off distance at which a superluminal pulse become subluminal, we solve for the distance $z$ at which the energy content associated with the superluminal and subluminal components of the pulse become equal. By scaling the input energy associated with the subluminal component (from Eq B.19) by its corresponding gain (obtained in Eq. B.13) and equating the result with the energy content of the superluminal part (from Eq. B.20) scaled by the respective gain (in Eq. B.17), the 
following equation can be derived:

$$
\begin{array}{r}
12 \delta \exp \left(\frac{z\left(\omega_{p, 1}^{2}+\omega_{p, 2}^{2}\right)}{8 c \delta}-\frac{1}{4} T^{2}\left(\omega_{0,1}-\omega_{c}\right)^{2}\right)=\frac{1}{2}\left(-6 \delta-\omega_{0,1}+\omega_{0,2}\right) \times \\
\exp \left(\frac{\delta\left(\omega_{0,1}+\omega_{0,2}\right)^{2} z\left[\frac{\omega_{p, 1}^{2}}{\omega_{0,1}^{2}\left(\omega_{0,1}-\omega_{0,2}\right)^{2}}+\frac{\omega_{p, 2}^{2}}{4 c}\right]}{4 c}\right) .
\end{array}
$$

By solving this equation for $z$, the cut-off distance $z_{c u t-o f f}$ can be expressed as:

$$
z_{\text {crossover }}=-\frac{2 c \delta \omega_{0,1}^{2}\left(\omega_{0,1}-\omega_{0,2}\right)^{2} \omega_{0,2}^{2}\left[T^{2}\left(\omega_{0,1}-\omega_{c}\right)^{2}+4 \log \left(-\frac{6 \delta+\omega_{0,1}-\omega_{0,2}}{24 \delta}\right)\right]}{2 \delta^{2}\left(\omega_{0,1}+\omega_{0,2}\right)^{2}\left(\omega_{0,2}^{2} \omega_{p, 1}^{2}+\omega_{0,1}^{2} \omega_{p, 2}^{2}\right)-\omega_{0,1}^{2}\left(\omega_{0,1}-\omega_{0,2}\right)^{2} \omega_{0,2}^{2}\left(\omega_{p, 1}^{2}+\omega_{p, 2}^{2}\right)} .
$$

In fact, this equation can also be solved for $\omega_{p}$ to find the medium dispersion strength at which an arbitrary pulse becomes subluminal (given a specific medium length $z$ ). In this case, assuming that $\omega_{p, 1}=\omega_{p, 2}=\omega_{p}$, then the cut-off dispersion strength can be evaluated using the following expression:

$$
\omega_{p_{\text {cut }} f f}=-\frac{\sqrt{c} \sqrt{\delta} \omega_{0,1}\left(\omega_{0,1}-\omega_{0,2}\right) \omega_{0,2} \sqrt{T^{2}\left(\omega_{0,1}-\omega_{c}\right)^{2}+4 \log \left(-\frac{6 \delta+\omega_{0,1}-\omega_{0,2}}{24 \delta}\right)}}{\sqrt{z\left[\omega_{0,1}^{2}\left(\omega_{0,1}-\omega_{0,2}\right)^{2} \omega_{0,2}^{2}-\delta^{2}\left(\omega_{0,1}+\omega_{0,2}\right)^{2}\left(\omega_{0,1}^{2}+\omega_{0,2}^{2}\right)\right]}} .
$$

Similarly, we may solve for the pulse width $T$ in terms of other parameters to obtain the following:

$T_{\text {cut }- \text { off }}=\frac{\sqrt{\frac{4 c \delta \omega_{0,1}^{2}\left(\omega_{0,1}-\omega_{0,2}\right)^{2} \omega_{0,2}^{2} \log \left(-\frac{6 \delta+\omega_{0,1}-\omega_{0,2}}{24 \delta}\right)+z\left[\delta^{2}\left(\omega_{0,1}+\omega_{0,2}\right)^{2}\left(\omega_{0,1}^{2}+\omega_{0,2}^{2}\right)-\omega_{0,1}^{2}\left(\omega_{0,1}-\omega_{0,2}\right)^{2} \omega_{0,2}^{2}\right] \omega_{p}^{2}}{\left[\delta^{2}\left(\omega_{0,1}+\omega_{0,2}\right)^{2}\left(\omega_{0,1}^{2}+\omega_{0,2}^{2}\right)-\omega_{0,1}^{2}\left(\omega_{0,1}-\omega_{0,2}\right)^{2} \omega_{0,2}^{2}\right] \omega_{p}^{2}}}}{\sqrt{-\frac{c \delta \omega_{0,1}^{2}\left(\omega_{0,1}-\omega_{0,2}\right)^{2} \omega_{0,2}^{2}\left(\omega_{0,1}-\omega_{c}\right)^{2}}{\left[\delta^{2}\left(\omega_{0,1}+\omega_{0,2}\right)^{2}\left(\omega_{0,1}^{2}+\omega_{0,2}^{2}\right)-\omega_{0,1}^{2}\left(\omega_{0,1}-\omega_{0,2}\right)^{2} \omega_{0,2}^{2}\right] \omega_{p}^{2}}}}$.

Finally, the expression for $z_{\text {cut-off }}$ (given in Eq. B.22), can be further simplified by omitting the term $\left[\log \left(-\frac{6 \delta+\omega_{0,1}-\omega_{0,2}}{24 \delta}\right)\right]$ in the numerator and the term $\left[2 \delta^{2}\left(\omega_{0,1}+\omega_{0,2}\right)^{2}\left(\omega_{0,2}^{2} \omega_{p, 1}^{2}+\right.\right.$ $\left.\left.\omega_{0,1}^{2} \omega_{p, 2}^{2}\right)\right]$ in the denominator. It can be shown that the contributions of both terms can be neglected (as long as $\delta<\frac{\omega_{0,2}-\omega_{0,1}}{10}$ and $\left.\left(\omega_{0,2}-\omega_{0,1}\right)<\frac{\omega_{0,1}}{10}\right)$. Therefore, Eq. B.22 can be re-written in the following simple form:

$$
z_{\text {cut }-o f f}=\frac{c \delta\left(\omega_{0,2}-\omega_{0,1}\right)^{2} T^{2}}{4 \omega_{p}^{2}}
$$




\section{Appendix C}

\section{Inverse Laplace Method}

In this section, the algorithm used in the numerical analysis based on the inverse Laplace method is presented. This is a fast and accurate numerical technique for computing the field in temporally dispersive Lorentzian media. For more details regrading the derivation of this algorithm, the reader is encouraged to visit ref [54].

For a signal that is propagating in a linear dispersive medium, the spectral amplitude $F(z, i \omega)$ satisfies Helmholtz equation

$$
\left[\nabla^{2}+k(i \omega)^{2}\right] F(z, i \omega)=0
$$

The general solution is given as

$$
F(z, i \omega)=F_{+}(i \omega) e^{i k(\omega) z}+F_{-}(i \omega) e^{-i k(\omega) z}
$$

The propagation vector $k(\omega)$ is expressed as $k(\omega)=\omega \frac{n(\omega)}{c}$. Assuming a Lorentzian dispersion with multiple resonances, the aforementioned expression can be written as:

$$
k(\omega)=\frac{\omega}{c} \sqrt{1 \pm \frac{\omega_{p, 1}^{2}}{\omega^{2}-\omega_{0,1}^{2}+2 i \delta \omega} \ldots \pm \frac{\omega_{p, n}^{2}}{\omega^{2}-\omega_{0, n}^{2}+2 i \delta \omega}} .
$$

Here, the '+' sign corresponds to the case of a gain medium while the '-' sign refers to absorption and the subscript ' $n$ ' denotes the number of medium resonances. Substituting $i \omega$ with $s, \mathrm{Eq}$ C.3 can be expressed as 


$$
k(i s)=\frac{i s}{c} \sqrt{1 \mp \frac{\omega_{p, 1}^{2}}{s^{2}-\omega_{0,1}^{2}+2 i \delta s} \ldots \mp \frac{\omega_{p, n}^{2}}{s^{2}-\omega_{0, n}^{2}+2 i \delta s}} .
$$

Accordingly, the propagating spectrum — for propagation in a double resonance Lorentzian medium with gain - is described as

$$
F(z, s)=F(0, s) e^{-s \frac{z}{c} \sqrt{1-\frac{\omega_{p, 1}^{2}}{s^{2}++\omega_{0,1}^{2} 2 \delta s}-\frac{\omega_{p, 2}^{2}}{s^{2}++\omega_{0,2}^{2} 2 \delta s}}} .
$$

For the specific case of a modulated Gaussian excitation with a pulse width $2 T$ and centered at a carrier frequency $\omega_{c}$, the initial spectral amplitude $F(0, s)$ is written as

$$
F(0, s)=\frac{1}{2 \sqrt{\pi}} T e^{\frac{-T^{2}}{4}\left(i s-\omega_{c}\right)^{2}}
$$

In order to numerically calculate the time response of the field $(f(z, t))$ at a fixed propagation distance $z$ and at each instant of time $t$, the following algorithm is used [54]

$$
f(z, t)=\frac{e^{a}}{t}\left[\sum_{n=1}^{k-1}(-1)^{n+1} \Im F\left(z, \frac{a+i(n-1 / 2) \pi}{t}\right)+\sum_{n=0}^{m} A_{m, n} F_{k+n}\right] .
$$

Here, $a$ is a constant parameter and the coefficients $A_{m, n}$ are obtained via the following recursive relation

$$
A_{i, j}=\sum_{k=j}^{i}\left(\begin{array}{l}
k \\
i
\end{array}\right)
$$

In order to calculate the field response at each instant of time, the coefficients $\left(A_{m, n}\right)$ are calculated from Eq C.8 and then substituted in the Eq C.7.

In summary, a fast and accurate algorithm to evaluate the field response of a dispersive medium has been presented. Such algorithm relies on the Laplace transform and can account for the high frequency oscillations (Sommerfeld precursors). For a detailed analysis of this algorithm, its limitations and approximations, the reader is encouraged to check ref [54]. 


\section{Bibliography}

[1] A. Sommerfeld, Annals of Phys. 44, 177, 1914.

[2] L. Brillouin, Annals of Phys. 44, 203, 1914.

[3] L. Brillouin, "Wave propagation and group Velocity," Academic, New York, 1960.

[4] C. G. B. Garrett and D. E. McCumber, "Propagation of a Gaussian light pulse through an anomalous dispersion medium," Physical Review A, Vol. 1, no. 2, pp. 305-313, 1970.

[5] S. Chu and S. Wong, "Linear pulse propagation in an absorbing medium," Physical Review Letters, Vol. 48, no. 11, pp. 738-741, 1982.

[6] B. Segard and B. Macke, "Observation of negative velocity pulse propagation," Physics Letters, Vol. 109A, no. 5, pp. 213-216, 1985.

[7] M. Mojahedi,E. Schamiloglu, F. Hegeler and K. J. Malloy, "Time domain detection of superluminal group velocity for single microwave pulses," Physical Review E, Vol. 62, 5758-5766, 2000.

[8] B. Ravelo, A. Prennec and M. Le Roy, "Synthesis of broadband negative group delay active circuits," IEEE International Microwave Symposium, Honolulu, HI, pp. 2177-2180, 2007.

[9] B. Ravelo, A. Perennec, M. Le Roy and Y.G. Boucher, "Active microwave circuit with negative group delay," IEEE Microwave and Wireless Components Letters, Vol. 17, no. 12, pp. 861-863, 2007.

[10] A. P. Barbero, H. E. Hernandez-Figueroa, and E. Recami, "Propagation speed of evanescent modes ," Physical Review E, Vol. 62, 8628, 2000. 
[11] D. Mugnai, A. Ranfagni and L. Ronchi, "The question of tunneling time duration: A new experimental test at microwave scale," Physics Letters A, Vol. 247, pp. 281-286, 1998.

[12] A. Enders, G. Nimtz, "Evanescent mode propagation and quantum tunneling," Physical Review E, Vol. 48, pp. 632-634, 1993.

[13] A. Ranfagni, D. Mugnai, P. Fabeni, and G. P. Pazzi, "Delay-time measurements in narrowed waveguides as a test of tunneling," Applied Physics Letters, Vol. 58, no. 7, pp. 774-776, 1991.

[14] D. Solli, R. Y. Chiao, and J. M. Hickmann, "Superluminal effects and negative group delays in electronics, and their applications," Physical Review E 66, 056601, 2002.

[15] J. Keaveney, I. G. Hughes, A. Sargsyan, D. Sarkisyan, and C. S. Adams, "Maximal refraction and superluminal propagation in a gaseous nanolayer," Physical Review Letters 109, 233001, 2012.

[16] M. D. Stenner, D. J. Gauthier and M. A. Neifeld, "The speed of information in a 'fast-light' optical medium," Nature, Vol. 425, pp. 695-698, 2003.

[17] L. J. Wang, A. Kuzmich and A. Dogariu, "Gain-assisted superluminal light propagation," Nature, Vol. 406, pp. 277-279, 2000.

[18] K. Kim, H. Seb Moon, C. Lee, S. Kim, and J. Bog Kim, "Observation of arbitrary group velocities of light from superluminal to subluminal on a single atomic transition line," Physical Review A 68, 013810, 2003.

[19] C. Spielmann, R. Szipocs, A. Stingl and F. Krausz, "Tunneling of optical pulses through photonic band-gaps," Physical Review Letters, Vol. 73, pp. 2308-2311, 1994.

[20] A. M. Steinberg, P. G. Kwiat and R. Y. Chiao, "Measurement of the single-photon tunneling time," Physical Review Letters, Vol. 71, 708, 1993.

[21] A. M. Steinberg, R. Y. Chiao, "Dispersionless, highly superluminal propagation in a medium with a gain doublet," Physical Review A, Vol. 49, 2071, 1991.

[22] R. Y. Chiao and A. M. Steinberg, Progress in Optics, Vol. 37, edited by: E. Wolf, Elsevier, pp. 347-406, 1997. 
[23] I. I. Smolyaninov, A. V. Zayats, A. Gungor, and C. C. Davis, "Single-photon tunneling via localized surface plasmons," Physical Review Letters, Vol. 88, 187402, 2002.

[24] S. Zhang, J. F. Chen, C. Liu, M. M. T. Loy, G. K. L. Wong, and S. Du, "Optical precursor of a single photon," Physical Review Letters 106, 243602, 2011.

[25] J. F. Woodley, M. Mojahedi, "Negative group velocity and group delay in left-handed media," Physical Review E, Vol. 70, 046603, 2004

[26] O. F. Siddiqui, S. J. Erickson, G. V. Eleftheriades, and M. Mojahedi, "Time-domain measurement of negative group delay in negative-fefractive-index transmission-line metamaterials," IEEE Transactions in Microwave Theory and Techniques, Vol. 52, no 5, pp. 1449-1454, 2004.

[27] Q. Li, Z. Zhang, J. Wang, M. Qiu, and Y. Su, "Fast light in silicon ring resonator with resonance-splitting," Optics Express, Vol. 17, Issue 2, pp. 933-940, 2009.

[28] C. Ciminelli, C. E. Campanella, F. DellOlio, and M. N. Armenise, "Fast light generation through velocity manipulation in two vertically-stacked ring resonators," Optics Express, Vol. 18, Issue 3, pp. 2973-2986, 2010.

[29] U. Vogl, R. T. Glasser, P. D. Lett, "Advanced detection of information in optical pulses with negative group velocity," Physical Review A, Vol. 86, 031806, 2012.

[30] E. L. Bolda, R. Y. Chiao and J. C. Garrison, "Two theorems for the group velocity in dispersive media," Physical Review A, Vol. 48, pp. 3890-3894, 1993.

[31] Klaas Wynne, "Causality and the Nature of Information," Elsevier, Optics Communications, Vol. 209, Issue 1-3, pp. 85-100, 2002.

[32] M. W. Mitchell and R. Y. Chiao, "Causality and negative group delays in a simple bandpass amplifier," American Journal of Physics, Vol. 66, no. 14, 1998.

[33] M. Tomita, H. Amano, S. Masegi, and A. I. Talukder, "Direct observation of a pulse peak using a peak-removed Gaussian optical pulse in a superluminal medium," Physical Review Letters 112, 093903, 2014.

[34] T. Martin and R. Landauer, "Time delay of evanescent electromagnetic waves and the analogy to particle tunneling," Physical Review A, 45, 2611, 1992. 
[35] G. Diener, "Superluminal group velocities and information transfer," Physics Letters A, Vol. 223, Issue 5, pp. 327-331, 1996.

[36] Z. Yang, "Physical mechanism and information velocity of fast light: A time-domain analysis," Physical Review A, Vol. 87, 023801, 2013.

[37] M. Pawlowski, T. Paterek, D. Kaszlikowski, V. Scarani, A. Winter, M. Zukowski, "Information causality as a physical principle," Nature Letters, Vol. 461, pp. 11011104, 2009.

[38] M. Mojahedi, K.J. Malloy, G. Eleftheriades, J. Woodley and R. Y. Chiao, "Abnormal wave propagation in passive media," IEEE Journal of Selected Topics in Quantum Electronics, Vol. 9, no. 1, pp. 30-39, 2003.

[39] A. Kuzmich, A. Dogariu, L. J. Wang, P. W. Milonni, and R. Y. Chiao, "Signal velocity, causality, and quantum noise in superluminal light pulse propagation," Physical Review Letters, Vol. 86, 3925, 2001.

[40] M. Z. Feng, W. Sorin, R. S. Tucker, "Fast light and the speed of information transfer in the presence of detector noise," IEEE Photonics Journal, Vol. 1, no3, pp. 213-224, 2009.

[41] L. Nanda, H. Wanare, and S. A. Ramakrishna, "Why do superluminal pulses become subluminal once they go far enough?," Physical Review A 79, 041806(R), 2009.

[42] A. H. Dorrah, L. Kayili, and M. Mojahedi, "Superluminal propagation and information transfer: a statistical approach in the microwave domain," Physics Letters A, Vol. 378, Issue 44, pp. 3218-3224, 2014.

[43] M. Ware, S. A. Glasgow, and J. Peatross, "Energy transport in linear dielectrics," Optics Express, Vol. 9, Issue 10, pp. 519-532, 2001.

[44] G. Denier, "Energy transport in dispersive media and superluminal group velocities," Physics Letters A, Vol. 235, pp. 118-124, 1997.

[45] E. L. Bolda, J. C. Garrison, and R. Y. Chiao, "Optical pulse propagation at negative group velocities due to a nearby gain line," Physical Review A 49, 2938, 1994.

[46] J. Peatross, S. A. Glasgow, and M. Ware, "Average energy flow of optical pulses in dispersive media," Physical Review Letters 84, $2370,2000$. 
[47] C. M. Balictsis and K. E. Oughstun, "Generalized asymptotic description of the propagated Field dynamics in Gaussian pulse propagation in a linear, causally dispersive medium," Physical Review E, Vol. 55, pp. 1910, 1997.

[48] K. E. Oughstun, G. C. Sherman, "Propagation of electromagnetic pulses in a linear dispersive medium with absorption (the Lorentz medium)," Journal of the Optical Society of America B, Vol. 5, No. 4, 817, 1988.

[49] K. E. Oughstun, G. C. Sherman, "Uniform Asymptotic Description of Electromagnetic Pulses in a Linear Dispersive Medium with Absorption (the Lorentz medium)," Journal of the Optical Society of America A, Vol. 6, No. 9, 1394, 1989.

[50] S. Shen, K. E. Oughstun, "Dispersive Pulse Propagation in a Double-Resonance Lorentz Medium," Journal of the Optical Society of America B, Vol. 6, No. 5, 948, 1989.

[51] K. E. Oughstun and C. M. Balictsis, "Gaussian pulse propagation in a dispersive, absorbing dielectric," Physical Review Letters 77, 2210, 1996.

[52] R. Safian, Mo. Mojahedi and C. D. Sarris, "Asymptotic description of wave propagation in an active Lorentzian medium," Physical review E, Vol. 75, Issue 6, 066611, 2007.

[53] C. M. Balictsis, "Unified asymptotic description of Gaussian pulse propagation of arbitrary initial pulse width in a Lorentz-type gain medium," Physical Review E 8\%, $013304,2013$.

[54] P. Wyns, D Foty, K. E Oughstun, "Numerical analysis of the precursor fields in linear dispersive pulse propagation," Journal of the Optical Society of America A, Vol. 6, Issue 9, pp. 1421-1429, 1989.

[55] A. Yariv, P. Yeh, "Photonics: Optical Electronics in Modern Communications," Oxford University Press, New York, pp. 501-507, 2007.

[56] L. Kayili, and M. Mojahedi, "Abnormal group delay and detection latency in communication systems," IEEE AP-S International Symposium and USNC/URSI National Radio Science Meeting,Canada, 2010. [DOI: 10.1109/APS.2010.5561972]

[57] Y. A. Vlasov1, M. O'Boyle1, H. F. Hamann1 and S. J. McNab1, "Active control of slow light on a chip with photonic crystal waveguides," Nature, 438, pp.65-69, 2005. 
[58] D. F. Phillips, A. Fleischhauer, A. Mair, R. L. Walsworth, and M. D. Lukin, "Storage of light in atomic vapor," Physical Review Letters 86, 783, 2001.

[59] R. S. Tucker, Pei-Cheng Ku, and C. J. Chang-Hasnain, "Slow-light optical buffers: capabilities and fundamental limitations," Journal of Light Wave Technology, Vol. 23, Issue 12, pp. 4046, 2005.

[60] J. B. Khurgin, "Optical buffers based on slow light in electromagnetically induced transparent media and coupled resonator structures: comparative analysis," Journal of the Optical Society of America B, Vol. 22, Issue 5, pp. 1062-1074, 2005.

[61] T. F. Krauss, "Why do we need slow light?," Nature Photonics 2, pp. 448-450, 2008.

[62] B. Corcoran, C. Monat, M. Pelusi, C. Grillet, T. P. White, L. O'Faolain, T. F. Krauss, B. J. Eggleton, and D. J. Moss, "Optical signal processing on a silicon chip at 640Gb/s using slow-light," Optics Express, Vol. 18, Issue 8, pp. 7770-7781, 2010.

[63] W. F. Silva, D. R. Solli, Adan J. Corcho, Dilson P. Caetano, and Jandir M. Hickmann, Fast Light, "Non-analytical points and the speed of information using pulses described by functions with compact support," Slow and Fast Light Conference, Washington DC, 2006. [DOI: http://dx.doi.org/10.1364/SL.2006.ME4]

[64] W. F. Silva, M. A. Alencar, D. P. Caetano, A. J. Corcho, and J. M. Hickmann, "Pulses with non analytical points described by functions with compact support: The information velocity in a fast-light medium," Slow and Fast Light Conference, Hawaii, United States, 2009. [DOI: http://dx.doi.org/10.1364/SL.2009.SMA6]

[65] R. Suzuki and M. Tomita, "Causal propagation of nonanalytical points in fast-and slow-light media," Physical Review A, Vol. 88, 053822, 2013.

[66] M. Tomita, H. Uesugi, P. Sultana, and T. Oishi, "Causal information velocity in fast and slow pulse propagation in an optical ring resonator," Physical Review A, Vol. 84, $043843,2011$. 